\title{
The Life of Christopher
}

\author{
Joshua Mugler \\ Hill Museum and Manuscript Library
}

(jmugler001@hmml.org)

\begin{abstract}
Christopher, a native of Baghdad who became patriarch of Antioch in about 349/960, was assassinated by Muslim rebels in 356/967 because of his loyalty to their Muslim ruler. When the Byzantines conquered Antioch two years later, his story was told in a variety of ways by those with different and competing interests. Christopher was mentioned in Byzantine histories and in Antiochian liturgies. However, by far the most extensive and detailed version of the story comes to us in the Life of Christopher, written by Ibrāhìm b. Yühannā, a Byzantine bureaucrat and translator who grew up in Antioch and knew Christopher when he, Ibrāhìm, was a young boy. The hagiography was originally composed in Greek and translated by its author into Arabic, but only the Arabic survives. Here I provide, for the first time, both a critical edition of the two known Arabic manuscripts and a full English translation. This text is a valuable testimony to Christian life in Antioch under both the Hamdānids and the Byzantines, and to the difficulties of life along the constantly shifting frontier of medieval northern Syria.
\end{abstract}

long with the history of Yahyā b. Sa ${ }^{\mathrm{c}} \overline{\mathrm{l}} \mathrm{d}$ al-Anțākī, with which it has a close and
complex relationship, the Life of Christopher is one of our most important testimo-
nies to Christian life in Hamdānid Syria. ${ }^{1}$ The Chalcedonian patriarch Christopher (d. 356/967), whose birth name was 'İsā, was born and raised in Baghdad, the Life tells us. He then moved to Syria and entered the Hamdānid bureaucracy, where he became a favorite of the emir Sayf al-Dawla (r. 333-56/944-67). In about 349/960, he became the patriarch of Antioch, the last to serve in that position before the Byzantine conquest of the city in 358/969. The Life was written by the Byzantine official Ibrāhīm b. Yūhannā. Ibrāhīm knew Christopher when the former was a young boy, but he did not write the Life until

1. Much of the information in this introduction, along with an earlier version of this translation, can also be found in my dissertation: Joshua Mugler, "A Martyr with Too Many Causes: Christopher of Antioch (d. 967) and Local Collective Memory” (PhD diss., Georgetown University, 2019).

(c) 2021 Joshua Mugler. This is an open access article distributed under the terms of the Creative Commons Attribution-NonCommercial-NoDerivatives License, which allows users to copy and distribute the material in any medium or format in unadapted form only, for noncommercial purposes only, and only so long as attribution is given to the original authors and source. 
his old age, around the late 410s/1020s. According to the heading of the text, he originally wrote it in Greek and then translated his own text into Arabic. Nevertheless, only the Arabic survives-and that only in two or possibly three manuscripts, one of which is currently unaccounted for.

The Hamdānids were one of many provincial dynasties that came to power as the central authority of the 'Abbāsid caliphate found itself stretched thin. ${ }^{2}$ They were members of the Arab tribe of Taghlib and originated from northern Mesopotamia. Nominally subordinate to the government in Baghdad, different branches of the family ruled Mosul and Aleppo from the early fourth/tenth century to the early fifth/eleventh. It seems that they had Shī'i sympathies, but they were not aggressively sectarian, and their allegiances sometimes shifted with the tides of political opportunism. ${ }^{3}$

Most of Christopher's adult life took place under the rule of the first Hamdānid emir of Aleppo, Sayf al-Dawla. Sayf al-Dawla made himself famous by patronizing some of the most prominent writers of the time, most notably the poets al-Mutanabbi (d. 354/965) and Abū Firās al-Ḥamdānī (320-57/932-68), a cousin of the emir. ${ }^{4}$ Other scholars criticized Sayf al-Dawla's harsh policies, but in the Life of Christopher we can see the protagonist receiving some of the same generous patronage that prompted so many celebrated poems. ${ }^{5}$

Unfortunately for Sayf al-Dawla, his rise to power in northern Syria coincided with a great expansion in Byzantine power, and during the reigns of Sayf al-Dawla and his descendants, the Byzantine Empire began to regain territory in this region for the first time since the first-/seventh-century Muslim conquests. Most of the Life's action takes place within this context, as Byzantine advances led to panic and rebellion in cities such as Antioch that grew ever nearer to the border. A major rebellion broke out in Antioch in 354/965, and this forms the pivot point of Christopher's patriarchate in the Life. ${ }^{6}$ Although Sayf al-Dawla was able to suppress the uprising, he had already begun to suffer from hemiplegia and was largely confined to his bed until his death in Șafar 356/February 967, at which point the brief power vacuum prompted further chaos in Hamdānid territory.

As the Hamdānids struggled with both internal and external pressures, the armies of Emperor Nikephoros II (r. 352-59/963-69) conquered Antioch in Dhū al-Hijja 358/October 969. Although Nikephoros was soon assassinated, his successor, John I (r. 359-65/969-76), thrilled to have regained control of the city that was once the great metropolis of Syria, quickly sought to reintegrate Antioch into the empire. As Gilbert Dagron puts it, "without Antioch, the 'reconquest' would win no more for Byzantium than some lands and cities;

2. Hugh Kennedy, The Prophet and the Age of the Caliphates: The Islamic Near East from the Sixth to the Eleventh Century (London: Routledge, 2016), 229-43; Clifford Edmund Bosworth, The Islamic Dynasties: A Chronological and Genealogical Handbook (Edinburgh: University Press, 1967), 49-50; Marius Canard, Histoire de la dynastie des H'amdanides de Jazîra et de Syrie (Paris: Presses universitaires de France, 1953).

3. Kennedy, Prophet, 231.

4. For selections from these poets and other authors that discuss Sayf al-Dawla and the events that took place under his rule, see Marius Canard, Sayf al Daula (Algiers: Editions Jules Carbonel, 1934).

5. Hugh Kennedy mentions the geographer Ibn Hawqal, who painted "a grim picture of overtaxation and exploitation"; see Kennedy, Prophet, 229.

6. Ibid., 241. 
with Antioch, it created a second pole, the virtual capital of a Roman Orient." John sent secular and ecclesiastical administrators from Constantinople and other parts of the empire to make Antioch Roman again. These administrators included the new patriarch, Theodore II, who is mentioned briefly in the Life. ${ }^{8}$ The empire undertook the translation of the liturgy of Constantinople into Syriac for use in the Church of Antioch, replacing local practices with those of the capital..$^{9}$ The Life also mentions several stages of imperial commemoration of Christopher, showing the new administration's determination to incorporate this local martyr into the new ecclesiastical order.

The Life was written after about fifty years of these imperial efforts and reflects some degree of local discontent with Roman control. The preceding rule of Sayf al-Dawla is presented in glowing terms, emphasizing the nuances and positive aspects of life with Muslims at a time when the empire viewed them primarily as foreign enemies. The text celebrates the autonomy and influence of the Antiochian Church under Muslim rule, in stark contrast to the situation of the church within the Byzantine Empire. Around the time that the Life was composed, in the late 410s/1020s, the empire's fortunes in the region began to turn again, and after decades of stagnation and lost ground, Antioch fell to the Seljuk Turks in 477/1084.

We know fairly little about the life of Ibrāhīm b. Yūhannā, author of the Life of Christopher, but we can make a general sketch. ${ }^{10}$ He was born in the early 340s/950s in Antioch to a prominent family with close connections to the church, and he was educated there under Patriarch Christopher. He spent his career within the Byzantine bureaucracy and attained the rank of prōtospatharios, along the way producing Arabic translations of some of the Greek works that had recently become available in Antioch thanks to the Byzantine reconquest of that city. These included works attributed to some of the greatest fourth-century CE Christian theologians, such as Pseudo-Dionysius the Areopagite and Ibrāhim's older contemporary Symeon Metaphrastēs. It is very likely that he was also closely involved with the imperial project of translating the Constantinopolitan liturgy into Syriac. Late in life, Ibrāhīm finally found the time to compose a hagiography of Christopher in Greek and Arabic-likely in conjunction with the celebration of Christopher's life under Patriarch Nicholas II in the 410s/1020s-as he had long intended to do. He must have died around $421 / 1030$ or shortly thereafter. Although he is not as famous as some other translators from middle Byzantine Antioch, most notably 'Abd Allāh b. al-Fạ̣l (d. ca. 444/1052), his life

7. Gilbert Dagron, “Minorités ethniques et religieuses dans l'Orient byzantin a la fin du Xe et au XI ${ }^{\mathrm{e}}$ siècle: L'immigration syrienne," Travaux et mémoires 6 (1976): 177-216, at 205.

8. Theodore is mentioned in $\S 18$ of the Life. For examples of Byzantines sent to administer the frontier cities, see Jean Darrouzès, ed., Épistoliers byzantins du Xe siècle (Paris: Institut français d'études byzantines, 1960).

9. Joseph Nasrallah, "La liturgie des patriarcats melchites de 969 à 1300," Oriens christianus 71 (1987): 156-81, at 156-59; Sebastian Brock, "Syriac Manuscripts Copied on the Black Mountain, near Antioch," in Lingua restituta orientalis, ed. Regine Schulz and Manfred Görg, 59-67 (Wiesbaden: Otto Harrassowitz, 1990), 66-67.

10. For more details on Ibrāhīm, see Joshua Mugler, "Ibrāhīm ibn Yūhannā and the Translation Projects of Byzantine Antioch," in Patristic Literature in Arabic Translations, ed. Barbara Roggema and Alexander Treiger, 180-97 (Leiden: Brill, 2020). 
offers an important window into the transition from Hamdānid Muslim rule to Byzantine Christian rule in Antioch, which he describes in the Life. ${ }^{11}$

Other testimonies to Christopher's story are far more limited in detail. They include Byzantine histories in Greek and entries in Antiochian liturgical calendars, both Syriac and Arabic. The history of Leo the Deacon, for example, claims that Antioch's "former Hagarene ruler killed the patriarch Christopher, an apostolic and divinely inspired man, by driving a javelin through his chest, bringing against the man the charge of reverence for Christ the Savior." ${ }^{12}$ This brief statement strips the story of any complexity and lends itself well to a polemic against the tyranny endured by Christians under Muslim rule. By contrast, the Life provides a much more nuanced picture of the situation.

The earliest and most reliable manuscript of the Life is Sinai Arabic 405 (S), which consists of the May-June volume of a full-year Menologion, a compilation of saints' lives according to the ecclesiastical calendar. ${ }^{13}$ The manuscript was copied at Mount Sinai in Kānūn al-Awwal 6843 Anno mundi, or Rabī' al-Thānī 735/December 1334. It was microfilmed by the Library of Congress in 1950, and the microfilm has now been made digitally available on the library's website. More recently, the manuscript has been digitized in color and made available through the website of UCLA's Sinai Manuscripts Digital Library project. ${ }^{14}$

The second manuscript (Z) was copied by Būlus b. al-Za'īm (d. 1079/1669), also known as Paul of Aleppo, son of Patriarch Makarios III (d. 1083/1672). It is a compilation of stories and other texts relevant to the history of Antioch. This manuscript was published with a French translation by Habib Zayat in 1952, but Zayat did not have access to the Sinai manuscript. Zayat says that Būlus's manuscript "is found in our possession" (se trouve en notre possession) but does not clarify this statement or explain where the manuscript is located. ${ }^{15}$ After his death in 1954, the matter became even more obscure, and there is now

11. For more on 'Abd Allāh, see Alexandre M. Roberts, Reason and Revelation in Byzantine Antioch: The Christian Translation Program of Abdallah ibn al-Fadl (Oakland: University of California Press, 2020).

12. Leo the Deacon, The "History" of Leo the Deacon: Byzantine Military Expansion in the Tenth Century, trans. Alice-Mary Talbot and Denis F. Sullivan (Washington, DC: Dumbarton Oaks Research Library and Collection, 2005), VI.6.

13. On the Antiochian Menologion, the large, full-year compilation of hagiographies that includes this manuscript, see Alexander Treiger, "Sinaitica (1): The Antiochian Menologion, Compiled by Hieromonk Yūhannā 'Abd al-Masī (First Half of the 13th Century)," Khristīanskĭ Vostok" 8 (2017): 215-52; Habib Ibrahim, "Liste des vies de saints et des homélies conservées dans les Ms. Sinaï Arabe 395-403, 405-407, 409 et 423," Chronos 28 (2018): 47-114. Treiger has recently redated the life of Yūhannā 'Abd al-Masīh, and thus the compilation of the Antiochian Menologion, to the early fifth/eleventh century. A note in the Menologion indicates that Yūhannā was a contemporary and acquaintance of Ibrāhīm, and in fact it is now clear that he must have been one of Christopher's disciples, mentioned at the end of his Life as "Anbā Yūhannā the Marvelous." See Alexander Treiger, "The Beginnings of the Graeco-Syro-Arabic Melkite Translation Movement in Antioch," Scrinium 16 (2020): 306-32, at 327-32. Habib Ibrahim has also edited an abridged version of the Menologion, published as Yūḥannā 'Abd al-Masīḥ al-Anțākī, Ma (īn al-ḥayāt:al-Markab al-sā'ir fị mīnā’ al-najāt, al-ma rūf bi-Kitāb al-dūlāb, ed. Habīib Ibrāhīm, 2 vols. (Beirut: Markaz al-Turāth al-`Arabī al-Masīhī, 2020-21).

14. https://sinaimanuscripts.library.ucla.edu/ (accessed July 12, 2021). The Library of Congress microfilm is available at https://www.loc.gov/item/00279389955-ms/.

15. Habib Zayat, "Vie du patriarche melkite d’Antioche Christophore († 967) par le protospathaire Ibrahîm b. Yuhanna: Document inédit du X $\mathrm{X}^{\mathrm{e}}$ siècle," Proche-Orient chrétien 2 (1952): 11-38, 333-66, at 13. 
no way to determine where this copy can be found. Fortunately Zayat's edition is available until Būlus's manuscript is uncovered again.

Alexander Treiger has recently discovered an additional copy of the Antiochian Menologion, presumably including the Life of Christopher, located in St. Petersburg: National Library of Russia, Arab. N.S. 92. ${ }^{16}$ This eight-volume set was copied in Damascus in 1261/1845 by Hannā b. Jirjis Șarrūf al-Dimashqī. Treiger believes that it was copied from a manuscript in the collection of the Greek Orthodox patriarchate in Damascus and that the antigraph was subsequently destroyed in the sectarian conflicts of 1276/1860, while Arab. N.S. 92 was brought to Russia by Porfiriu Uspenskĭ. It will hopefully be available for study soon, but I have not been able to consult it in the process of preparing this edition.

An edition of the text was published by Ignatius Dick in 1997, but even though it uses both the Sinai manuscript and Zayat's text, it is not a critical edition. ${ }^{17}$ It lacks a thorough presentation of the variants between the manuscripts and instead presents a seamless text that leans heavily on the Sinai manuscript, bringing in occasional corrections from Zayat. Furthermore, it is extremely difficult to obtain a copy of this book outside the Middle East. Finally, Sofia Moiseeva published a Russian translation of Zayat's text in 2013, incorporating only those Sinai variants that were found in a short excerpt published in a 1979 article by Joseph Nasrallah. ${ }^{18}$

Zayat explains that his copy of the text was damaged and that Būlus himself was copying from a badly damaged copy. Būlus writes:

Be aware, my brother, that at the beginning of the patriarchate of my father, I found this marvelous and unique account at the end of a very old book, badly written, deprived of diacritical points, nearly illegible, and gnawed by mites; numerous passages had also disappeared. But in all the Arab countries with their monasteries and churches, I have not been able to find a second copy of it. I believed it necessary to reproduce it here, because it is so precious. ${ }^{19}$

Thus Zayat's text, although extremely valuable as a second witness alongside Sinai Ar. 405, has suffered greatly from the ravages of time.

The historical sections of the Life bear an obvious resemblance to those found in Yahyā b. Sa'īd al-Anțākī's history, known as the Dhayl. Scholars have typically explained the similarity by claiming that the Life is "the source" of these elements of the Dhayl. ${ }^{20}$ In most cases, these scholars worked only with Zayat's published text, in which the verbatim

16. Treiger, "Beginnings," 332.

17. Ighnāṭiyūs Dīk, Sīrat al-bațiyark Kharīsțūfūrus al-Anțākī șadīq Sayf al-Dawla (Aleppo: n.p., 1997).

18. Ibrāhīm b. Yūḥannā, "Zhitie antiokhiı̌skogo patriarkha Khristofora," trans. S. A. Moiseeva, in ArabyKhristiane v istorii i literature Blizhnego Vostoka, ed. N. G. Golovnina, 28-61 (Moscow: PSTGU, 2013); Joseph Nasrallah, "Deux auteurs melchites inconnus du Xe siècle," Oriens christianus 63 (1979): 75-86, at 79-82. The translation is reprinted in Moiseeva's 2015 monograph; see Sofia A. Moiseeva, Arabskaia mel'kitskaia agiografiia IX-XI vekov (Moscow: PSTGU, 2015), 142-75.

19. Zayat, "Vie," 15.

20. Ibid., 15; John Harper Forsyth, "The Byzantine-Arab Chronicle (938-1034) of Yahyā b. Sa'īd al-Anțākī” (PhD diss., University of Michigan, 1977), 182-86. 
borrowing from one text to the other is extensive. However, when examining the $\mathrm{S}$ version of the Life, the relationship between the two works becomes far murkier: there is greater divergence between the two extant manuscripts in the historical sections than there is in other sections, and the most significant differences concern the summaries and verbatim equivalents of al-Anțākī's account, which are consistently found in $\mathrm{Z}$ but have no parallelor are phrased very differently-in $\mathrm{S}^{21}$

The $\mathrm{Z}$ variants contain nothing that is not found in al-Anțākì's text, but $\mathrm{S}$ does contain information not found elsewhere. For example, when Christopher is assassinated, $Z$ states that the murderers threw his head into a public bath furnace-as described in al-Anțākìs Dhayl-whereas S claims instead that his body was dragged around the city on a ladder, a detail found in no other source. ${ }^{22}$ This makes it highly unlikely that the recension contained in $\mathrm{S}$ was produced by editing an original text closer to Z. Instead, it seems likely that the redactor of Z's version edited an earlier text while copying and summarizing material from the Dhayl. The fact that $\mathrm{S}$ is an earlier manuscript witness than $\mathrm{Z}$ is circumstantial evidence for this position. The editing may have been carried out by Būlus b. al-Za'ìm or by one of his predecessors. Perhaps it was done to make the text more historically rich and-occasionally-more straightforwardly comprehensible, as the literary style of $\mathrm{S}$ is sometimes rather difficult to follow. ${ }^{23}$ Counterintuitively, therefore, much of the material shared between the two texts was actually added from al-Anțākīs text to Ibrāhīm's by a later editor, not borrowed from Ibrāhīm's work by al-Anțākī himself.

Other, less substantial variants between $\mathrm{S}$ and $\mathrm{Z}$ involve the replacement of archaic or obscure words in $\mathrm{S}$ with their more current equivalents in $\mathrm{Z}$ or simply glossing obscure terms, as $\mathrm{Z}$ does with the term $b \bar{a} q \bar{u} l \bar{a} .{ }^{24}$ With all this in mind, it seems nearly certain that $\mathrm{S}$ is closer to the original text of the Life than $\mathrm{Z}$ is, especially in the historical sections in which $\mathrm{Z}$ relies heavily on borrowing from al-Anțākì's work. As a result, my approach in the edition has been to prefer the reading of $S$ in most cases. This approach yields a more

21. The one exception to this rule occurs in $\$ 17$ of the Life, where S recounts in detail that Peter the Stratopedarkhēs was attacking Aleppo while the Syrian gang was trying to return the captive Ibn Mānik to him. Here S follows closely the description of events in al-Anțākīs Dhayl, whereas Z simply states that the Syrians "brought him [Ibn Mānik] to the stratopedarkhēs" and ignores the attack on Aleppo altogether. Compare Yahyā b. Sa'īd al-Anțākī, "Histoire de Yahya-Ibn-Sa“ïd d'Antioche, continuateur de Sa“ïd-Ibn-Bitriq," ed. and trans. I. Kratchkovsky and A. Vasiliev, Patrologia orientalis 18, no. 5 (1924): 699-833, at 823-24.

22. Ibrāhīm, Life, §15; al-Anțākī, "Histoire," 809.

23. For example, $\mathrm{S}$ has a tendency to describe major characters and refer to them by epithets without revealing their names until the end of their part in the story, if at all. $\mathrm{Z}$ rearranges the text to introduce characters by name, generally when they are first mentioned. This difference helps explain some of the quirks noted by Moiseeva. For example, Moiseeva notes an instance in which an entire set of sentences is repeated; it now seems clear that the first occurrence of these sentences is borrowed from al-Anțāki and placed earlier in Z, whereas the second occurrence, found later in both manuscripts, reflects Ibrāhīm's original composition. The borrowing from al-Anțākī thus explains the repetition. See Sofia A. Moiseeva, "The Early Melkite Arabic Hagiography (IXth-XIth Centuries): Evolution of the Literary Style," Parole de l'Orient 39 (2014): 33-56, at 51; Ibrāhīm, Life, §17; al-Anțākī, "Histoire," 814.

24. Ibrāhīm, Life, §17. 
accurate picture of the text composed by Ibrāhīm b. Yūhannā in the fifth/eleventh century while still giving access to the redactor's later work in the apparatus.

Beyond variants, $\mathrm{S}$ fills most of the gaps present in the damaged text of $\mathrm{Z}$. More noteworthy than any of these variants and lacunae, however, is the presence in $S$ of a two-page ethical preface that has been completely excised from Z. In this introductory section, Ibrāhīm lays out a vision of human nature and divine justice that should shape the way we read the text as a whole. He emphasizes the human capacity to improve and to turn away from a past life of sin-or even just a past life of ethical mediocrity-and argues that we, like God, "should view all those whom we see according to the way they look at the end of their days, whether they have been good and righteous or have returned to goodness and righteousness after straying far away." 25 This is the context in which Ibrāhim introduces his protagonist, Christopher, whose early life in the luxurious context of government employment was suspect in ethical terms, but who turned from that life to one of asceticism and generosity when he became the patriarch of Antioch. The inclusion of this preface and the benefits to be gained from critically comparing the two manuscripts will greatly improve our understanding of this valuable text.

In this edition and translation, I have largely kept to the standard spelling of modern Arabic with respect to issues such as the presence or absence of dots on the letters $\checkmark$ and

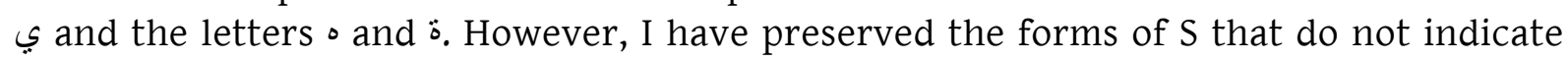
the hamza, or glottal stop. I have also standardized proper names, which often differ slightly between the two manuscripts and from one occurrence to the next within a single manuscript, and I have not indicated their numerous small variants. Otherwise, all variants have been noted in the apparatus to the edition; in the translation, I have mentioned only those variants that seem especially noteworthy or entail significant changes in meaning. The notes to the translation also clarify historical and narrative details that are relevant for understanding the text. I use w (in the edition) and S (in the translation) to refer to Sinai Arabic 405, and $j$ and $Z$ to refer to the lost manuscript edited by Zayat in 1952. Folio numbers in both edition and translation refer to the folios of $S$, from fol. $111 \mathrm{v}$ to fol. $131 \mathrm{r}$.

25. Ibrāhīm, Life, preface. 


\section{Arabic Edition}

\section{1v بسم الاب والابن والروح القدس الالاه الواحد}

اليوم الثالث والعشرون من ايار

قصة سيرة البطريرك على انطاكية الثهيد خريسطوفورس وشهادته بها

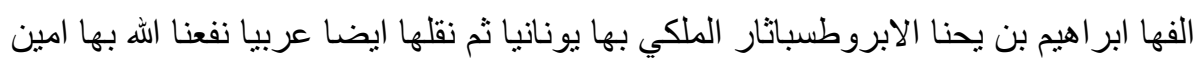

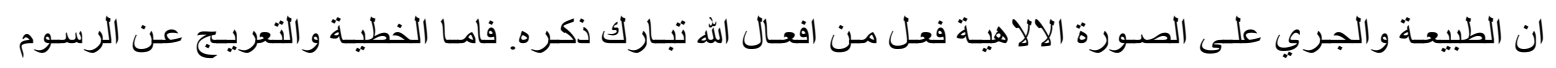
الموجودة في الصورة فذللك فعل من افعال الثرير.

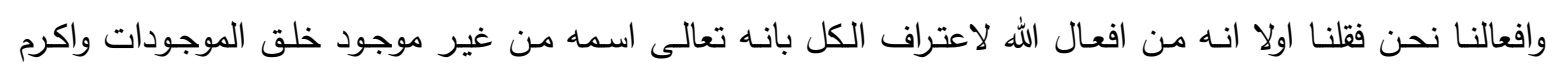

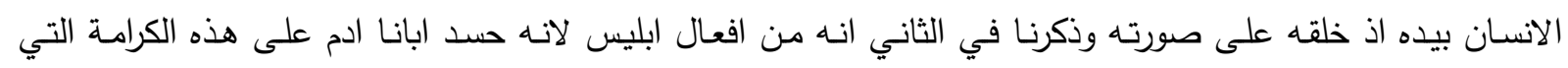

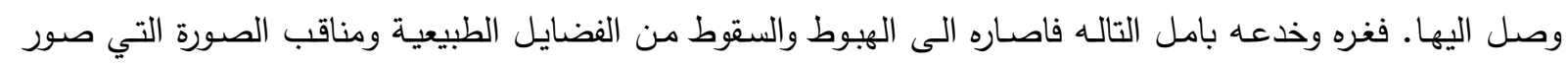

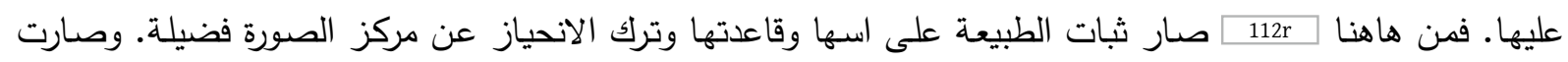

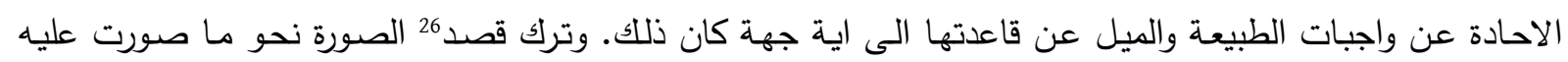
وتوجهها الى ضد ذلك نقيصة واديات الطيعة ورذيلة.

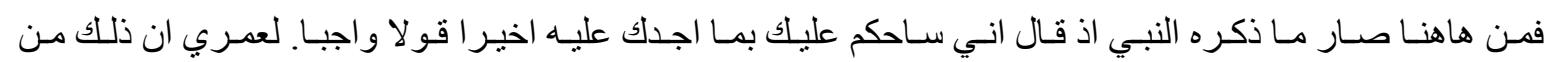

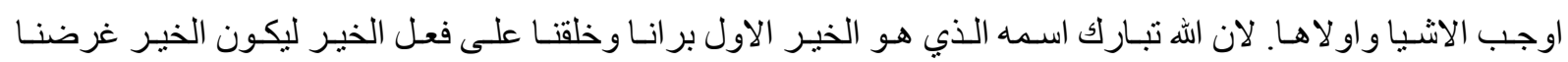

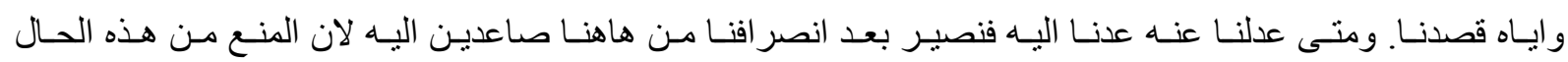

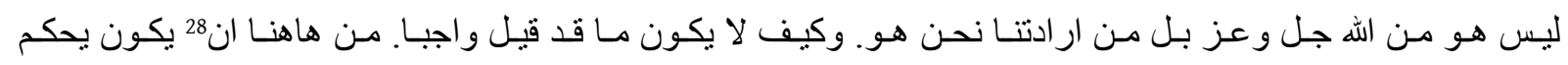

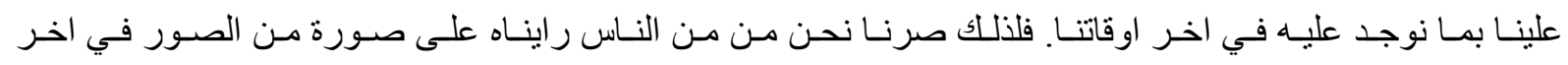

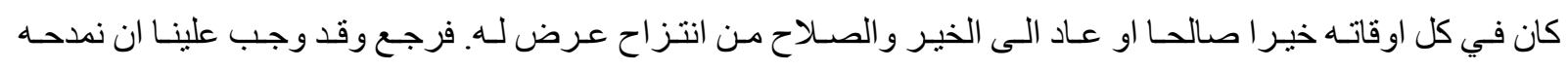

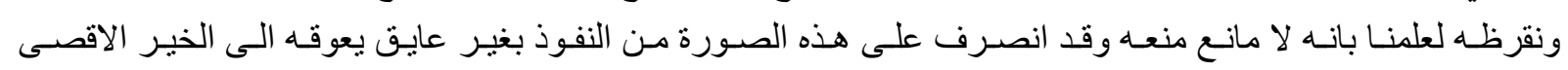
و الماتور الابعد.

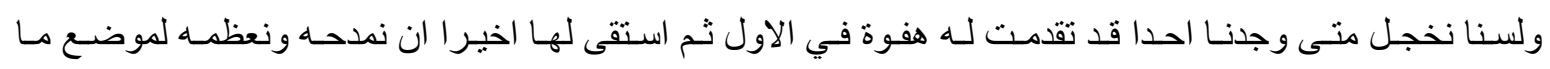

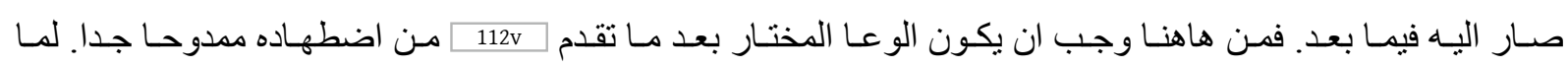

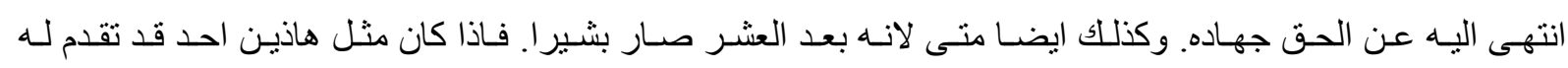

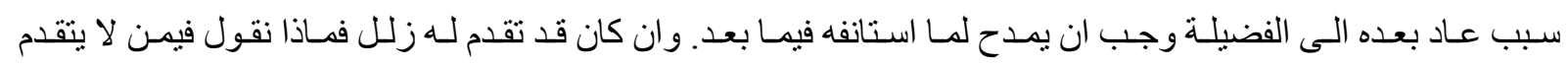

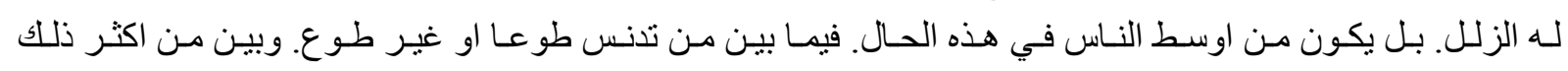

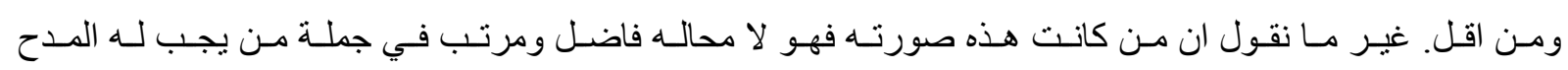
الجزيل.

\section{6. قصد: صححته؛ قصر: س س س \\ 27. نقيصة: صححته؛ نقيضة: سن س س س}

28. ان: صححته؛ ان يكون ما قد قيل واجبا من هاهنا ان: س س سل 
[1] و الذذي نعرفـه بهذه الصـورة فهو خريسطوفورس البطريـرك العظيـم والثـهيد الكريـم فيمـا تقدم لـه مـن عطايـاه

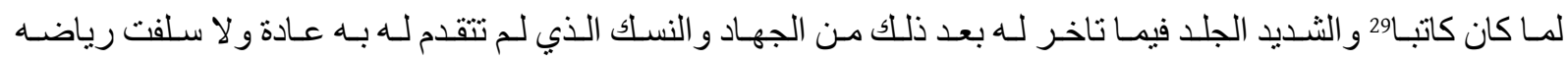

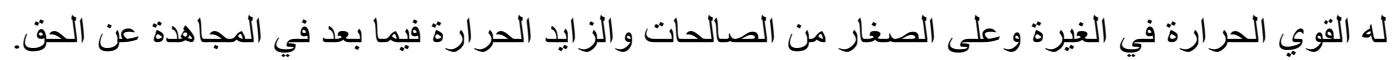

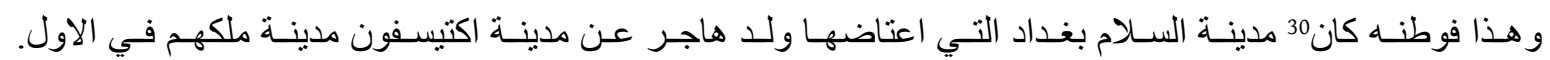

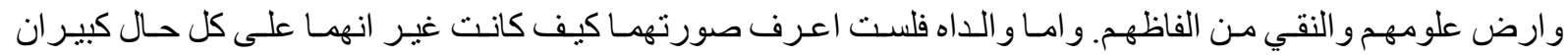

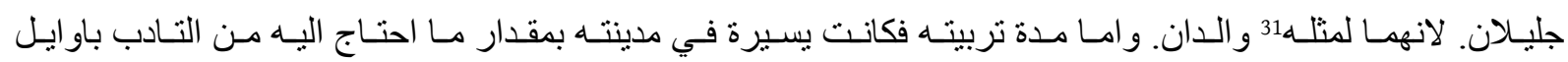

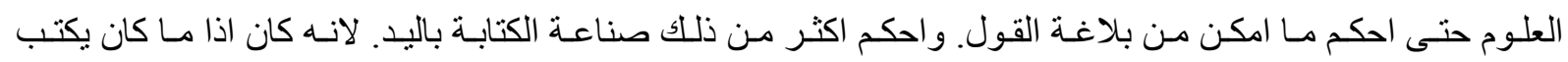

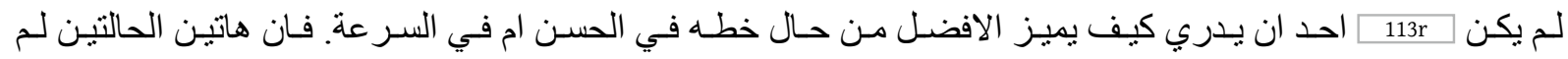

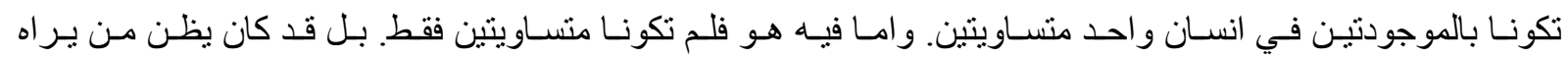

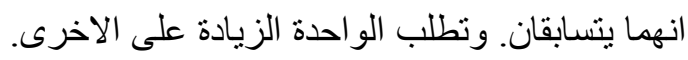

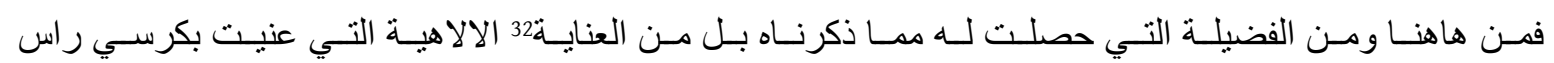

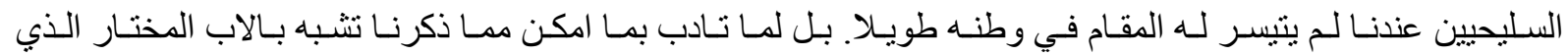

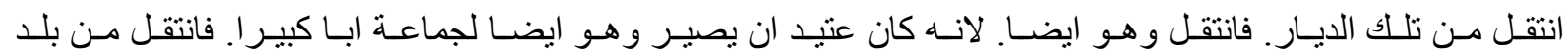

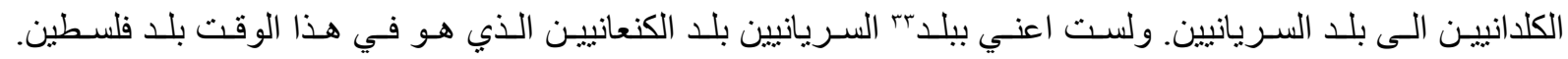

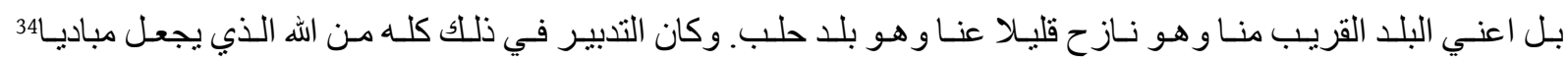

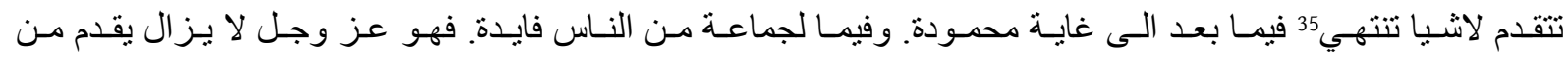
الصغار مقدمات لكبار لا تخطر فيما قبل بو هم.

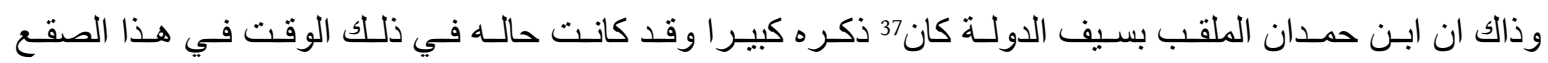

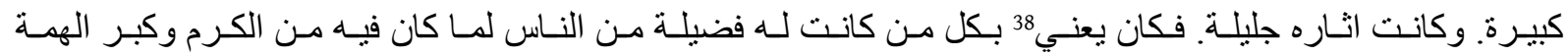

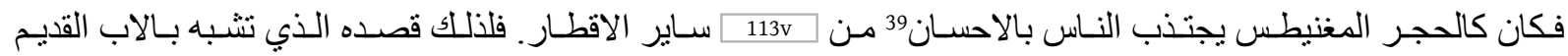

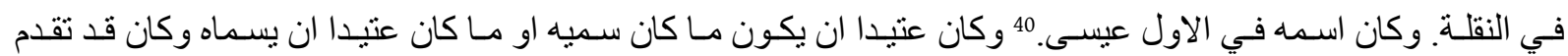

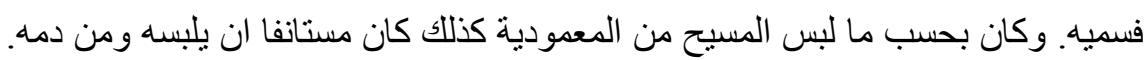

$$
\begin{aligned}
& \text { 29. بسم الاب . . . كان كاتبا: س؛ هذا المغبوط السعيد و البطريرك المعظم و المكرم الثهيد: ز }
\end{aligned}
$$

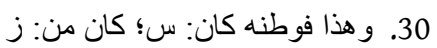

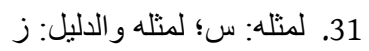

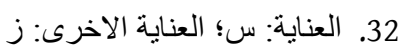

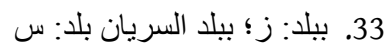

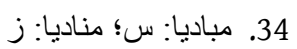

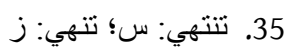

$$
\begin{aligned}
& \text { 36. بوهم: س؛ بذهن: ز سلعي: }
\end{aligned}
$$

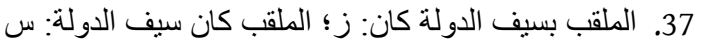

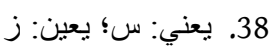

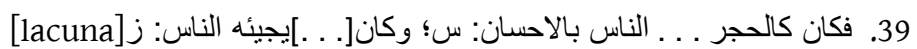

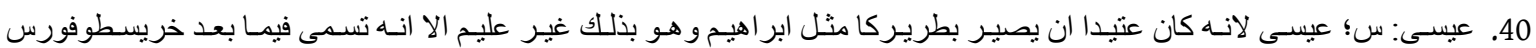




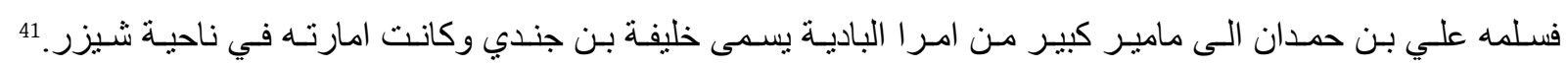

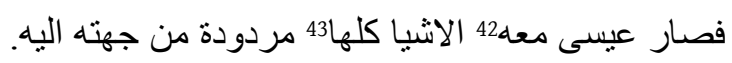

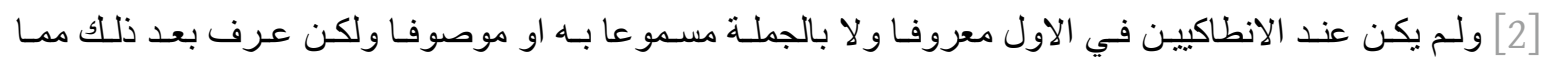

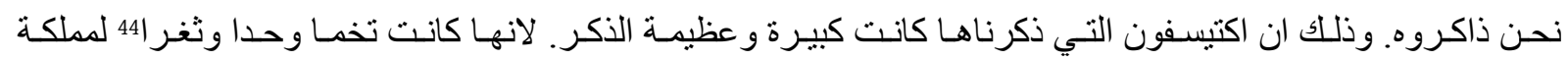

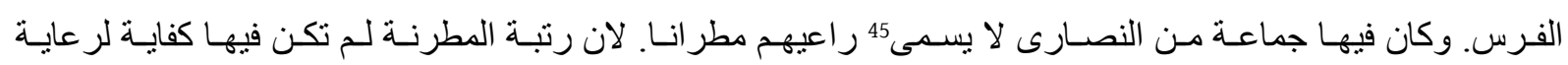

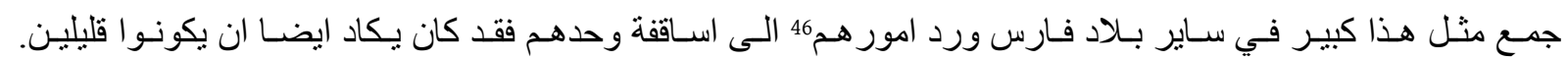

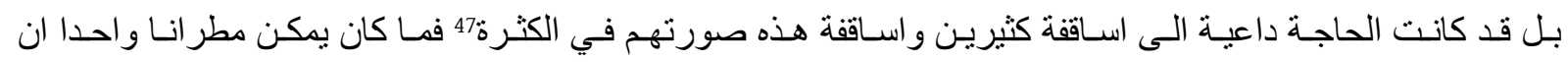

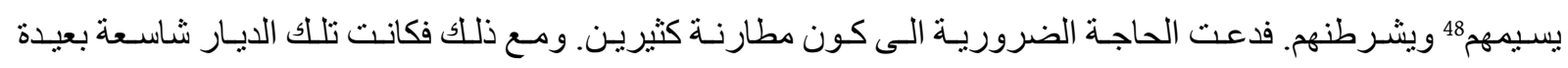

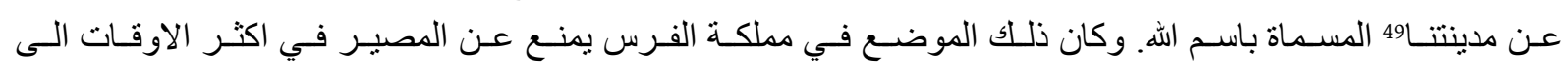
114r

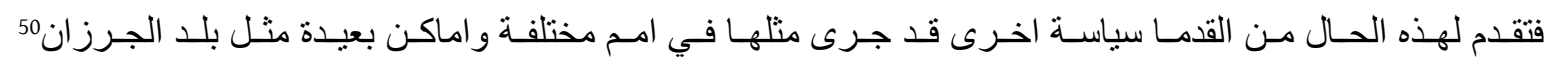

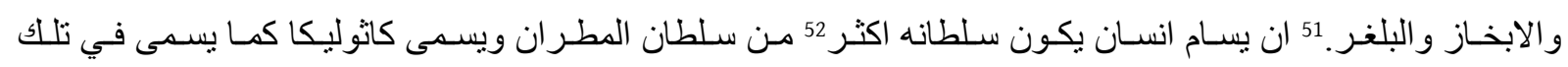

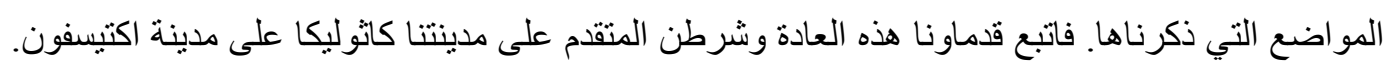

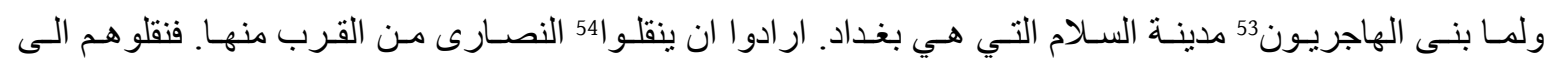

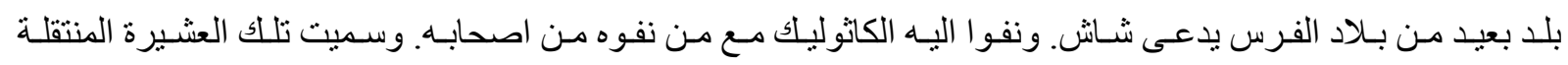
جماعة الروم. فوجب ان يكون اسم جمعهم هذا الاسم.

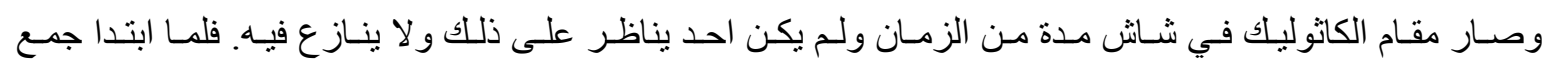

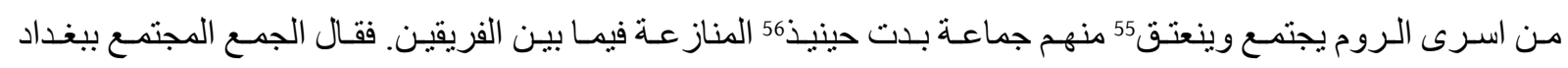

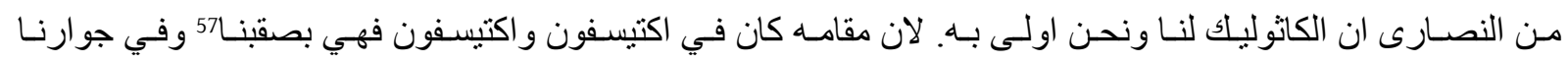

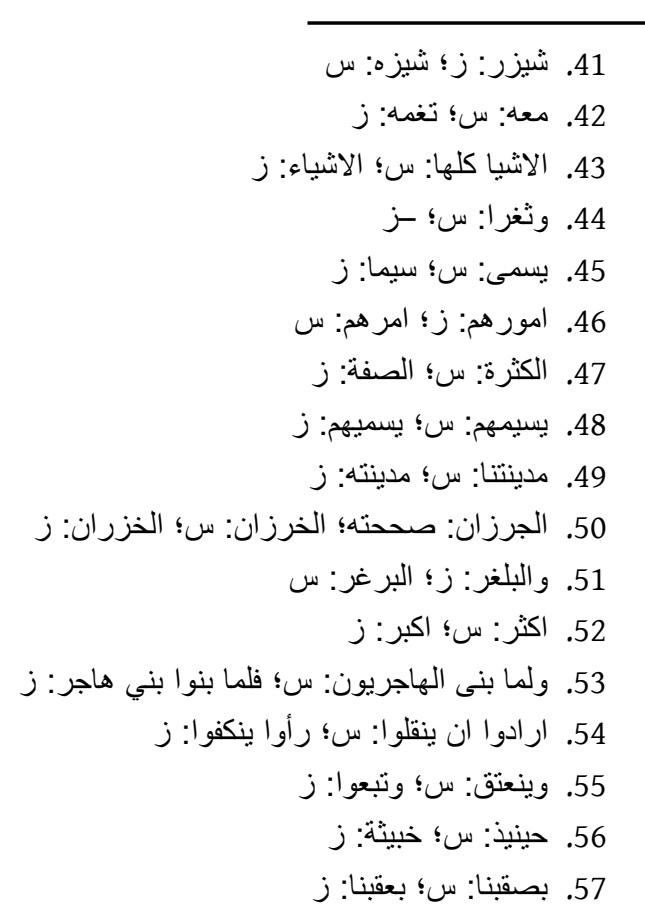

Al-`Ușūr al-Wusțā 29 (2021) 


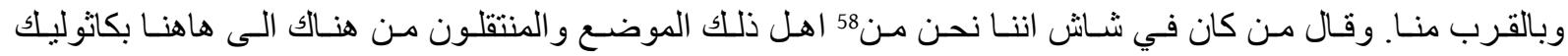

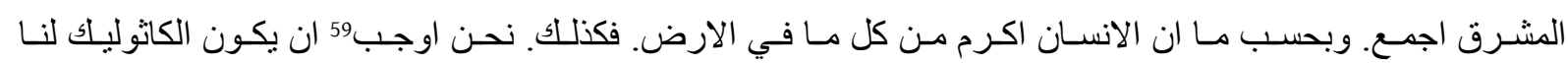

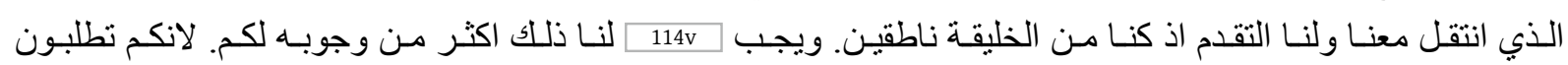
ان يكون لكم التقدم من الحجارة والتراب.

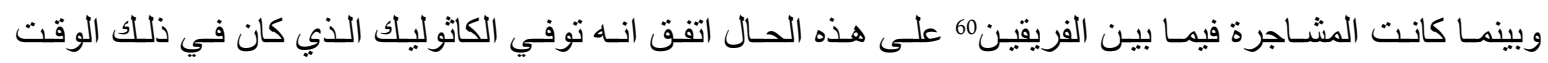

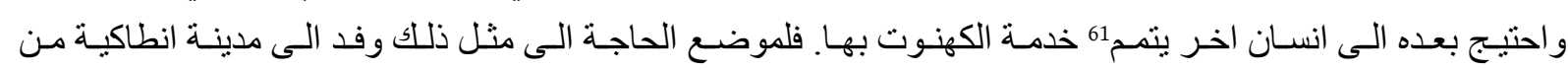

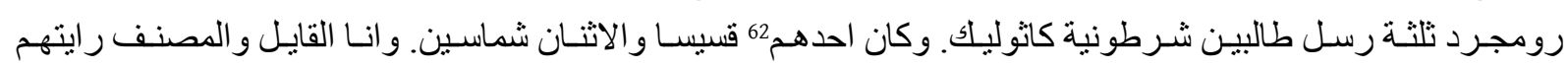

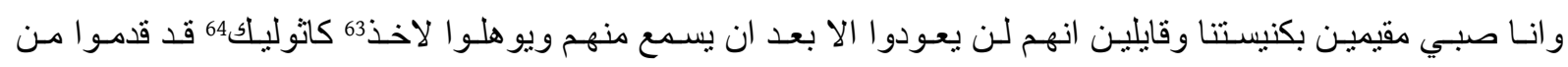

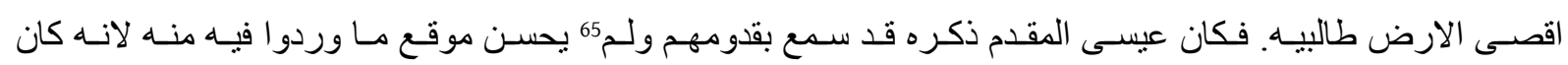

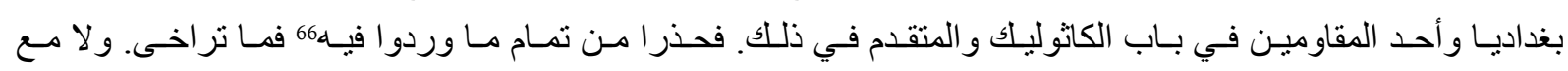

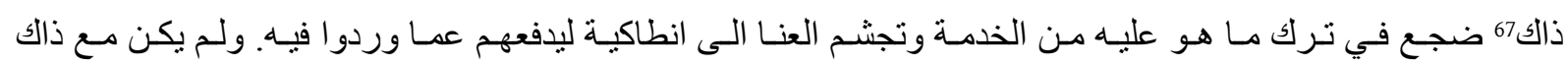

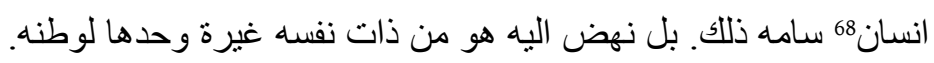

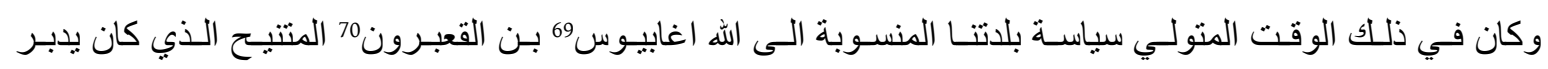

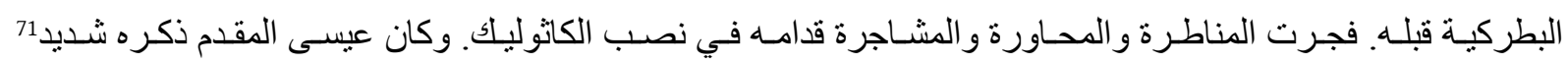

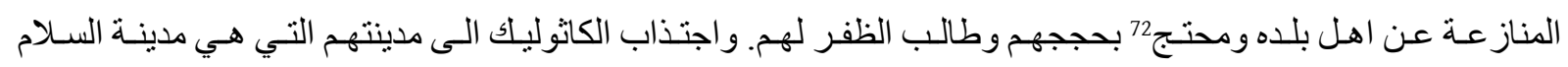

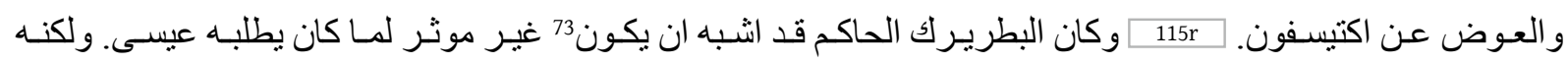

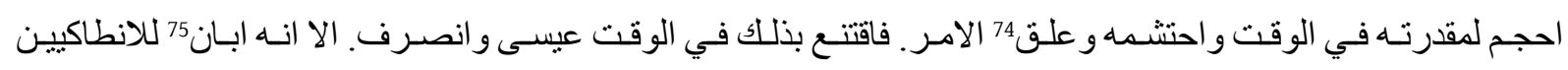
منه انه رجل كبير جلد وان له غيرة شديدة في امور الكنيسة.

$$
\begin{aligned}
& \text { 58. 5ن: ز؛ هم: س } \\
& \text { 59. 58. اوجب: س؛ و واجب: زئ ز } \\
& \text { 60. الفريقين: س؛ الفئتين: ز ز }
\end{aligned}
$$

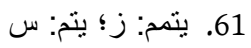

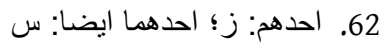

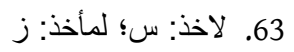

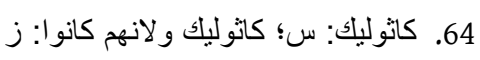

$$
\begin{aligned}
& \text { 65. 65. ولم: س؛ وليس: ز ز }
\end{aligned}
$$

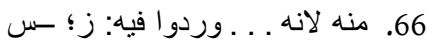

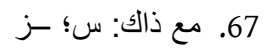

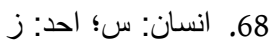

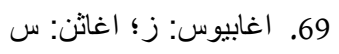

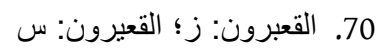

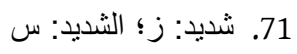

$$
\begin{aligned}
& \text { 72. }
\end{aligned}
$$

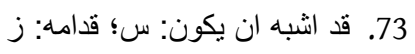

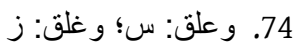

$$
\begin{aligned}
& \text { 75. ابان: س؛ بان: ز ز }
\end{aligned}
$$


[3] فلم يكن الا هنيهـة مـن الزمـان حتـى76 توفي البطريـرك. وصـار اهتمـام الانطاكيين في اختيـار بطريرك وراع

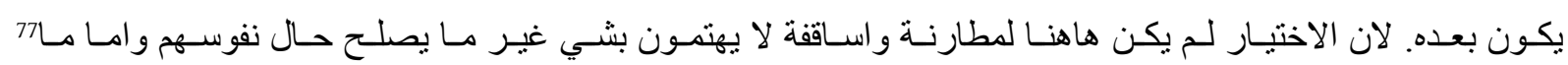

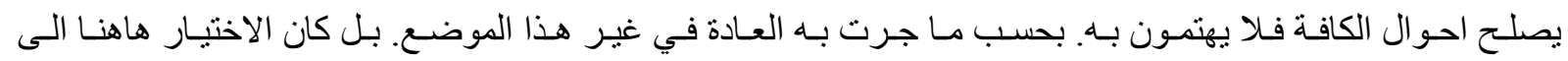

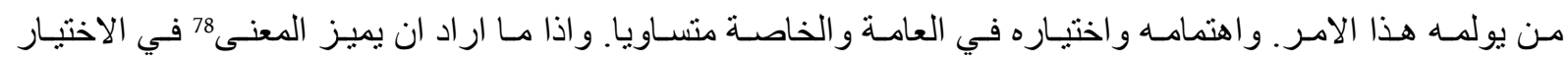

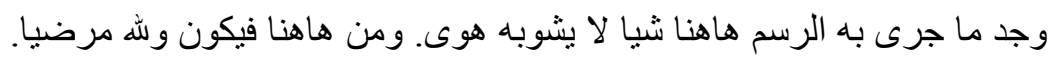

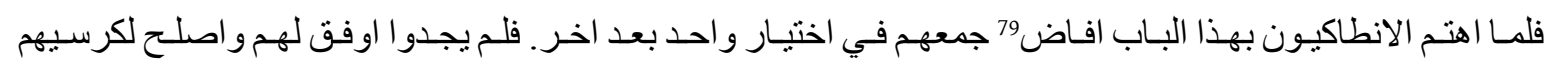

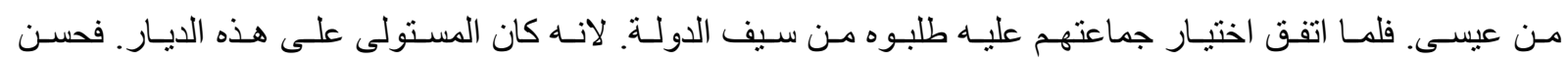

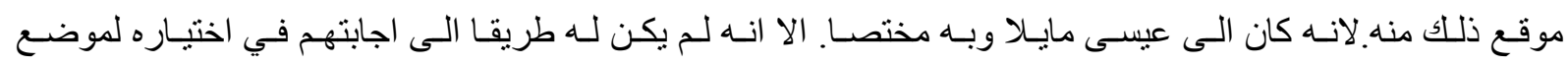

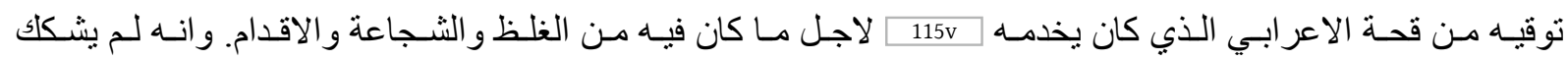

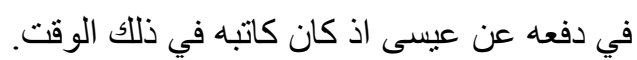

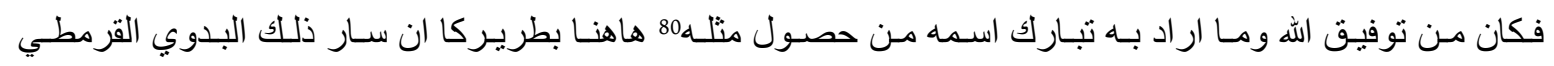

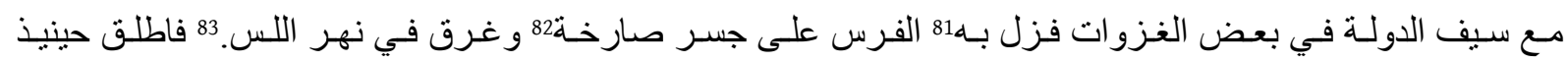

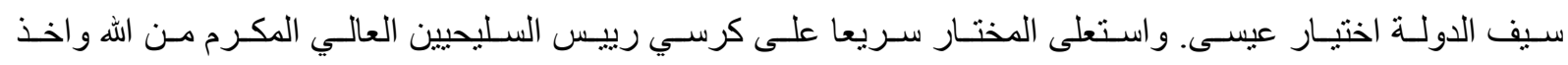

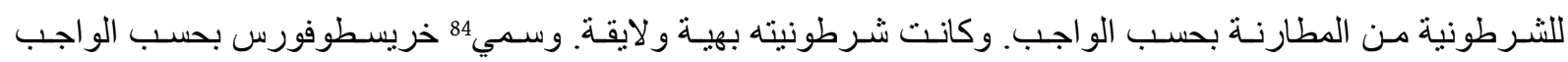

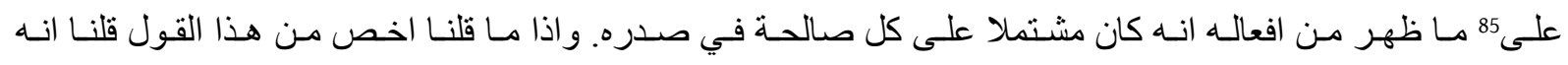
كان قد ضم المسيح في قلبه.

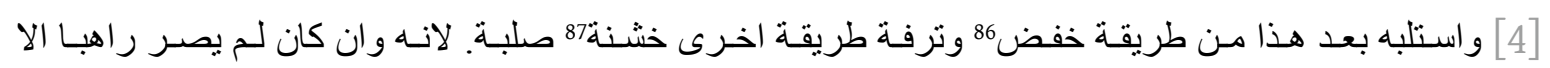

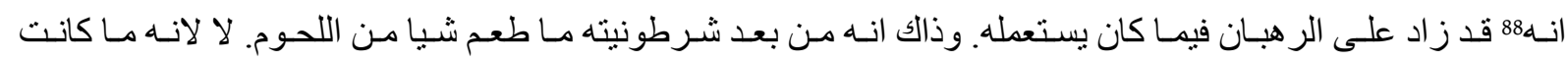

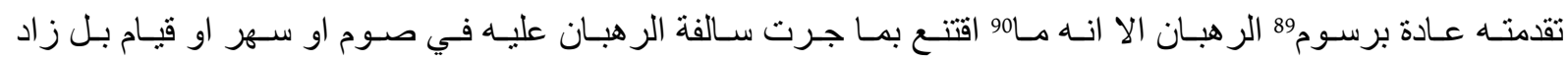

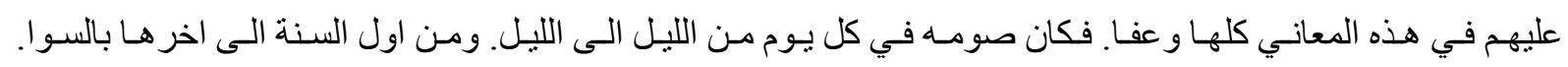

\author{
76. حتى: س؛ -ز \\ 77. 78.

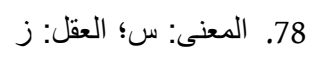

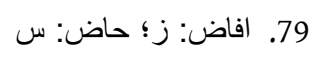

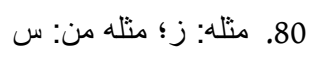

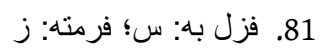

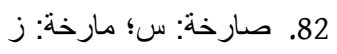

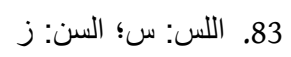

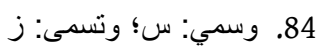 \\ 85. الواجب على: س؛ - -ز

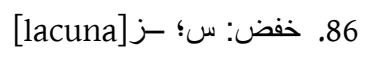

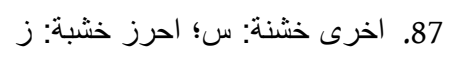 \\ 88. الا انه: ز؛ سـ

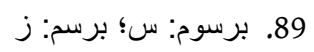 \\ 90. الا انه ما: ز؛ سـ برس
}

Al-`Ușūr al-Wusțā 29 (2021) 


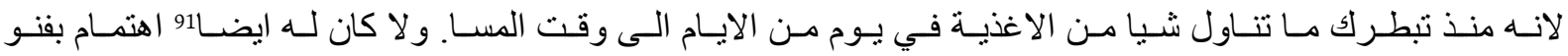

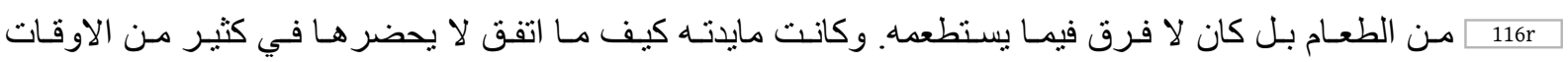

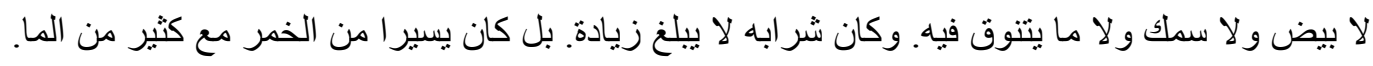

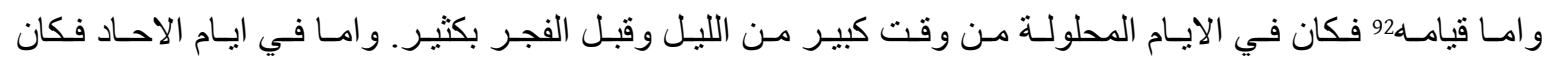

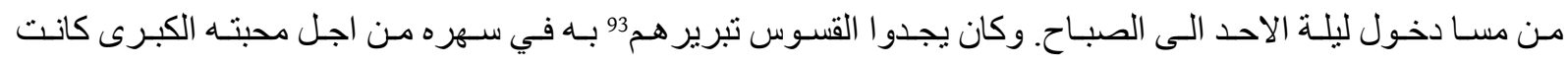

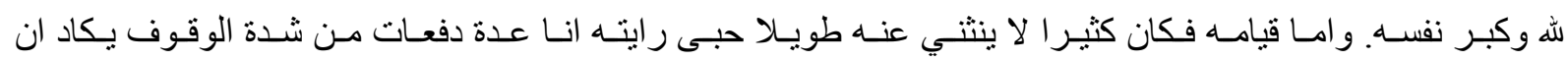

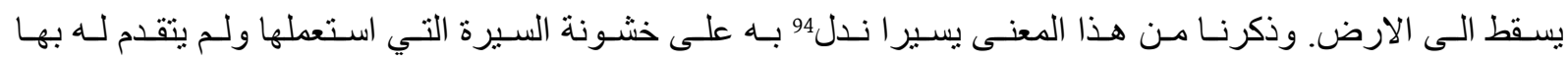

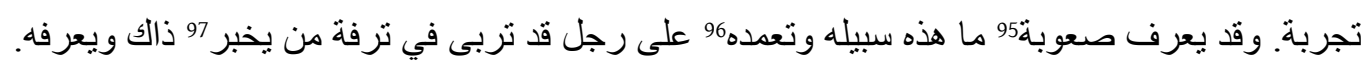

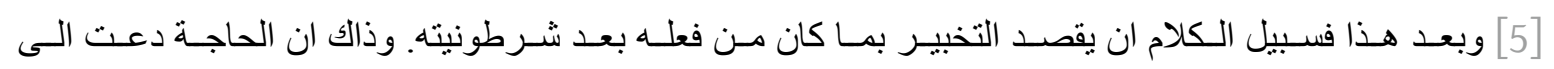

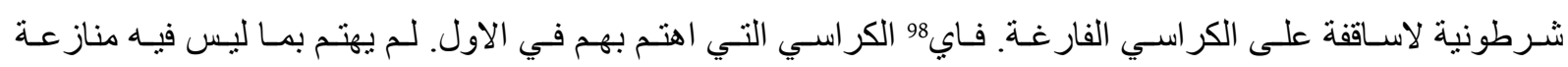

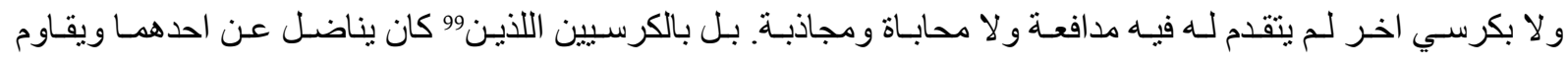

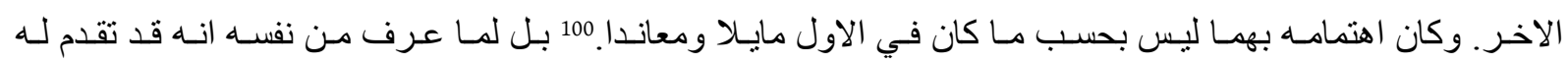

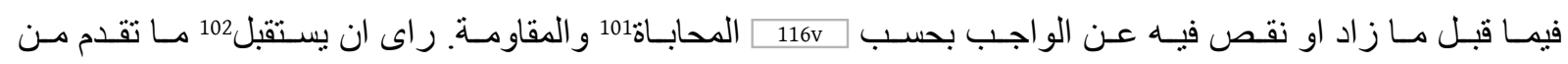

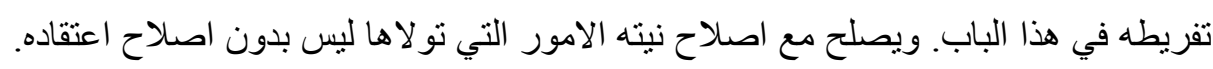

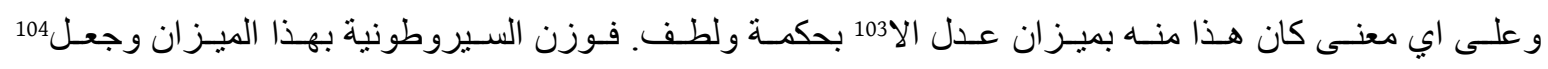

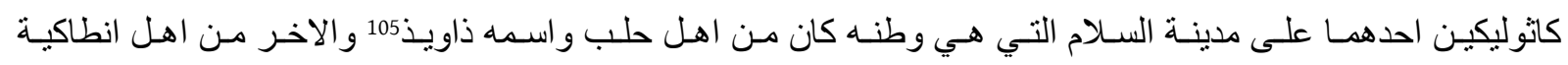

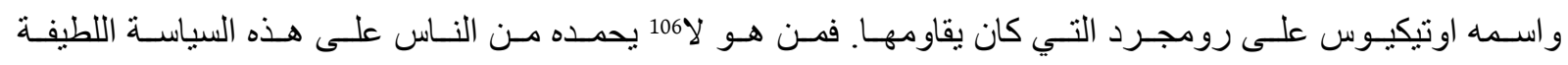

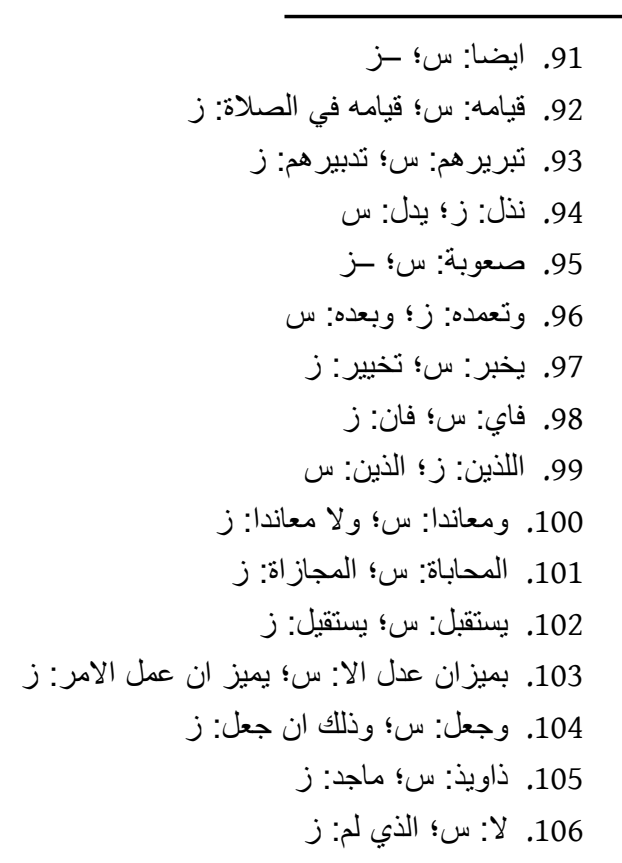

Al- 'Ușūr al-Wusțā 29 (2021) 


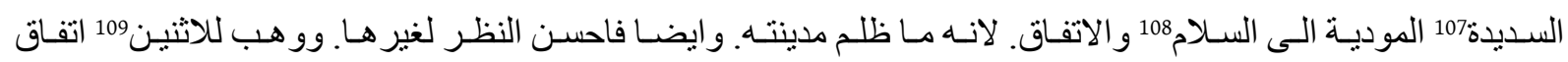
النفوس و البعد من الخصايم و المناز عات.

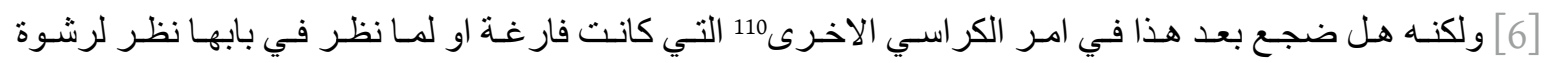

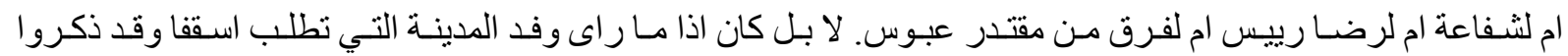

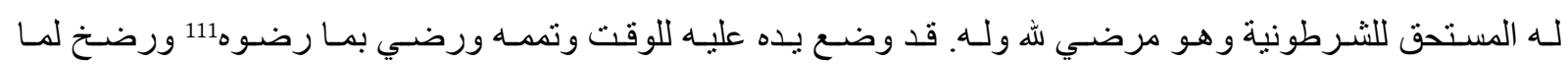

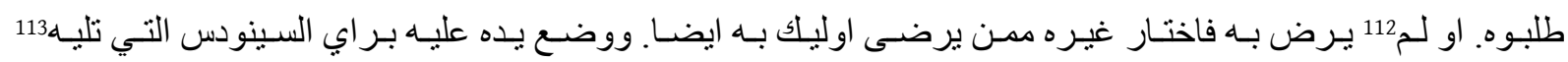

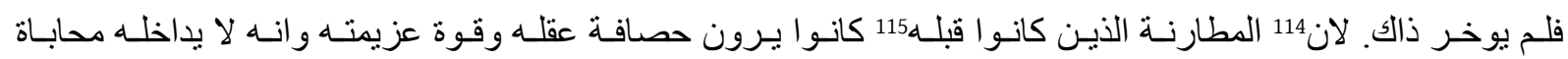
ولا ير غب في عطا ولا رشوة. فكيف كان يمكنهم ان يخالفوه في شي مما يو مي 116 اليه. 117

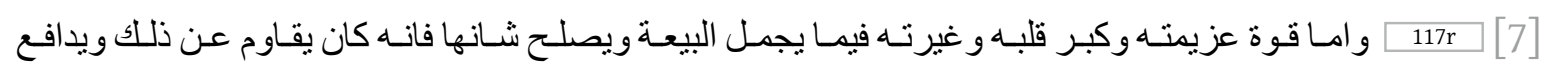

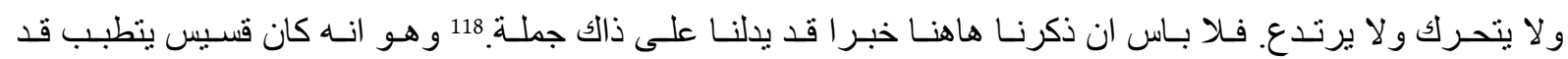

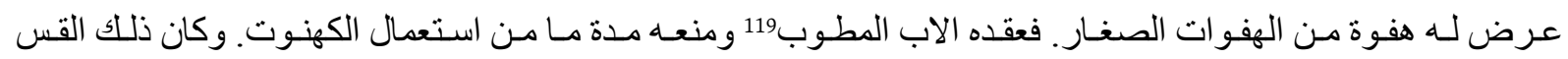

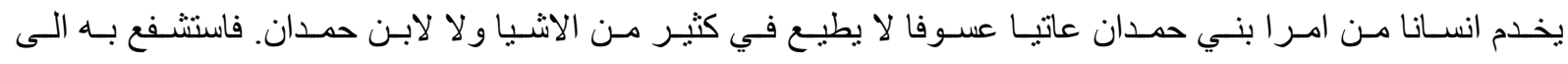

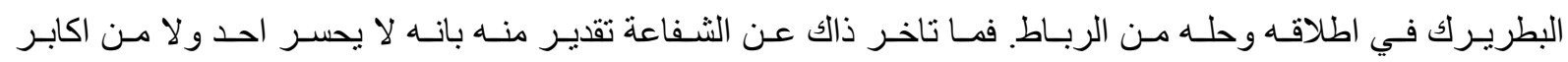

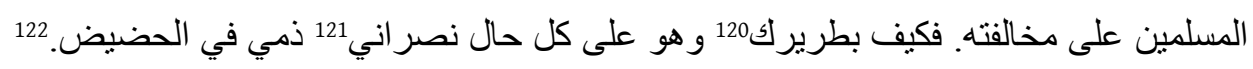

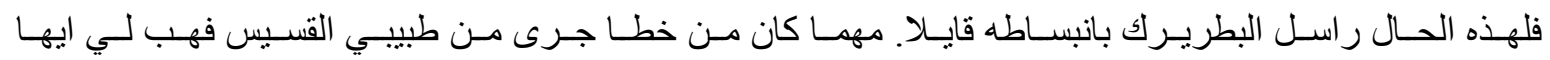

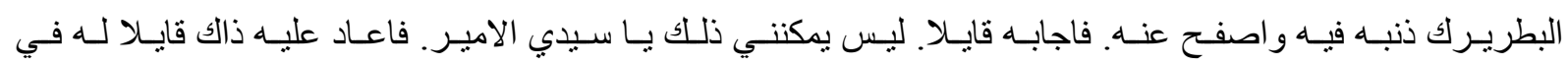

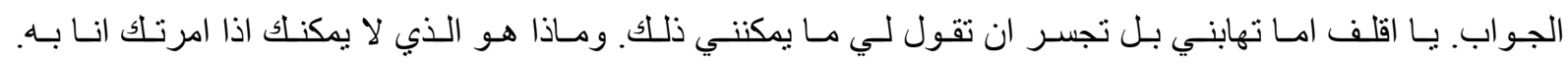

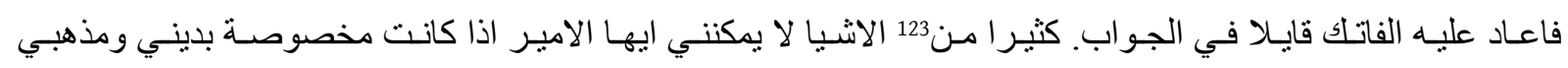

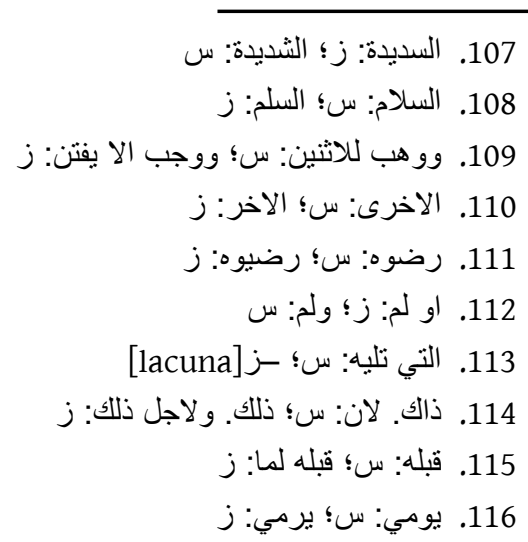

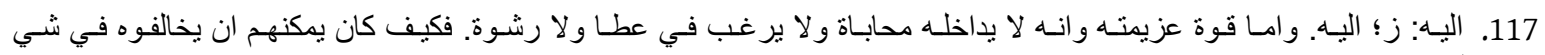

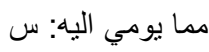

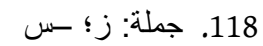

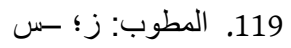

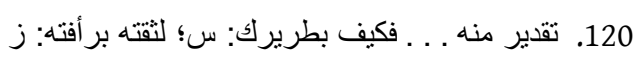

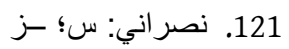

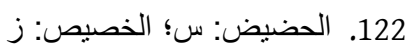

123. الفاتك قايلا . . . كثبر ا من: س؛ القائل قائلا: الحو ادث كثيرة من هذه: ز

Al-'Ușūr al-Wusțā $29(2021)$ 


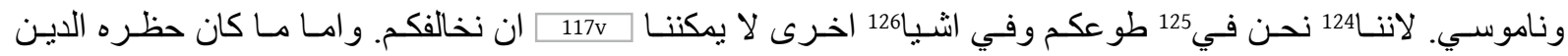

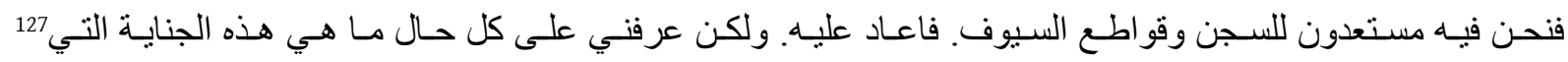

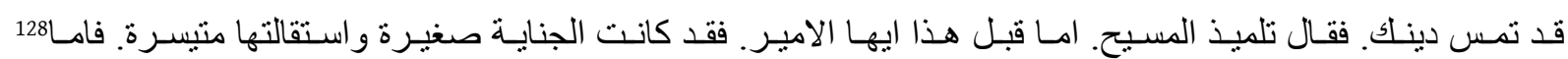

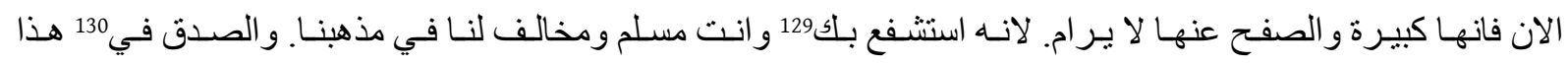

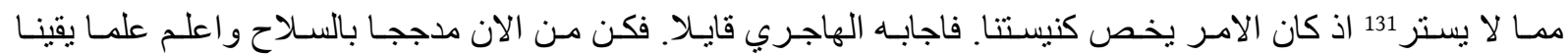
انك ستموت. وذاك اني اخذ راسك ولو كان في حضن الامبر الاكبر.

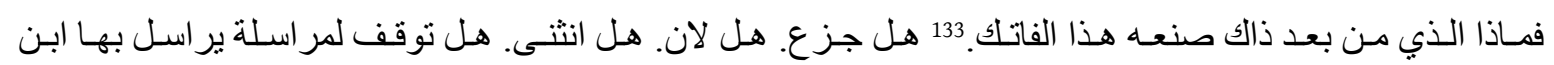

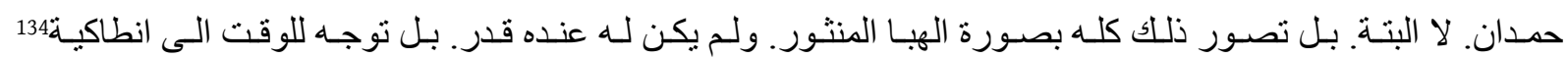

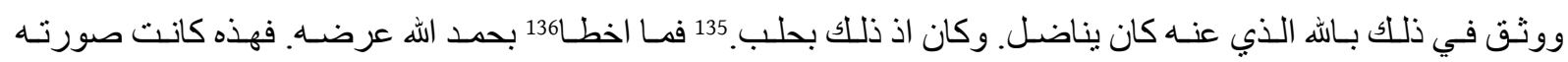

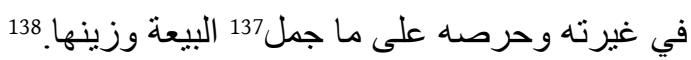

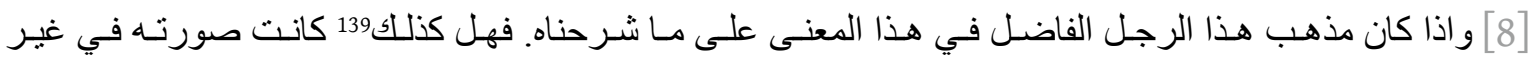

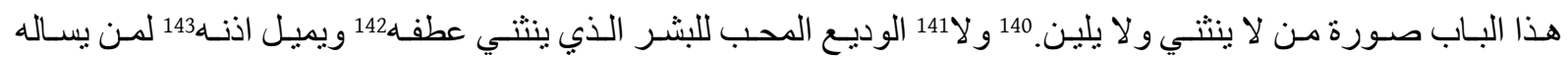

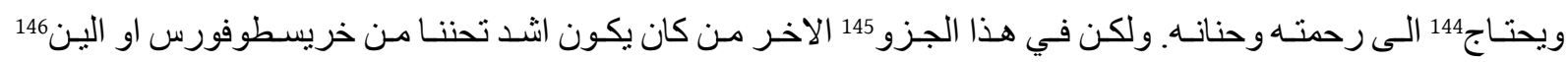

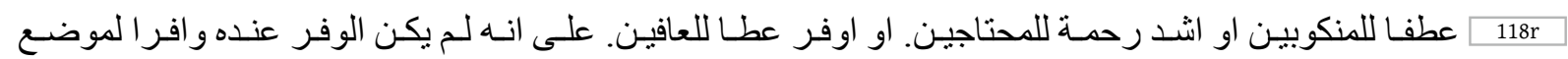

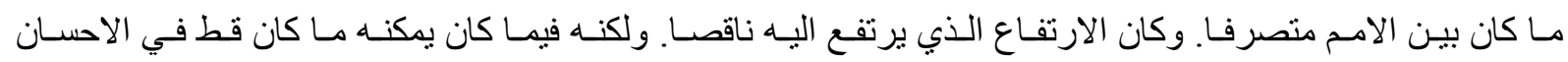

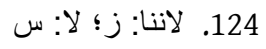

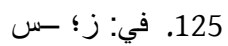

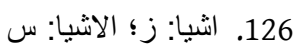

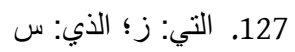

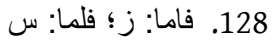

$$
\begin{aligned}
& \text { 129. لانه استشفع بك: س؛ سك لاني استشنع به: ز ز } \\
& \text { 130. في: س؛ - سز لز }
\end{aligned}
$$

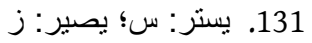

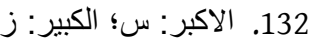

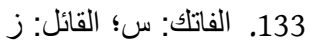

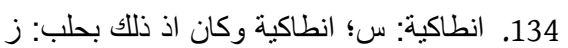

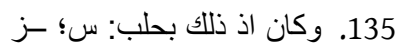

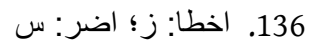

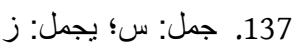

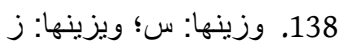

$$
\begin{aligned}
& \text { 139. فهل كذلك: س؛ فئ فئلك: ز ز ز }
\end{aligned}
$$

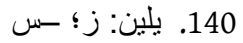

$$
\begin{aligned}
& \text { 141. و لا: س؛ ولا وكيف كان يليق انه من اعماله تلميذ المسيح: ز }
\end{aligned}
$$

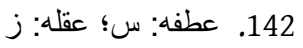

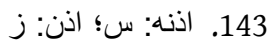

$$
\begin{aligned}
& \text { 144. ويحتاج: س؛ ما يحتاج: ز زل زل }
\end{aligned}
$$

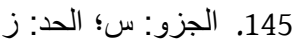

$$
\begin{aligned}
& \text { 146. او الين: ز؛ و اللين: س }
\end{aligned}
$$


مقصـر ا و الارفـاد و العنايــة والبـذل ليسس دفعـة واحـدة وغيـر دفعـات و لا متفو اتـا وغيـر متصـل اذ كان يتسـع الامـكان ويساعد اليسر 147 ولا يقاوم العشر.

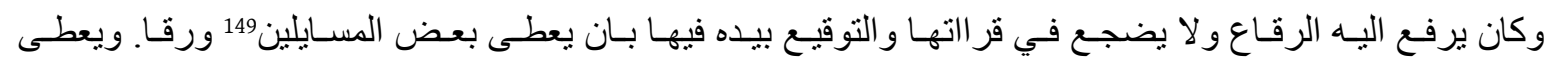

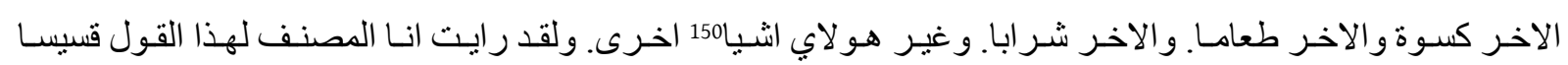

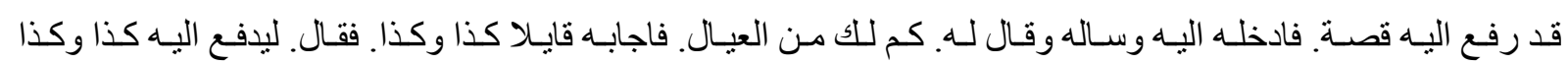

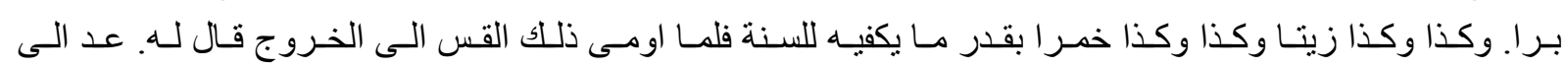

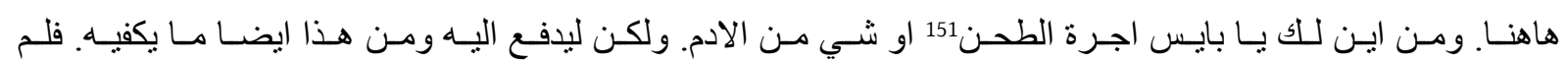
يتركه152 القديس ينصرف ومن اينى قام له بجميع ما يقنعه لسنته كلها.

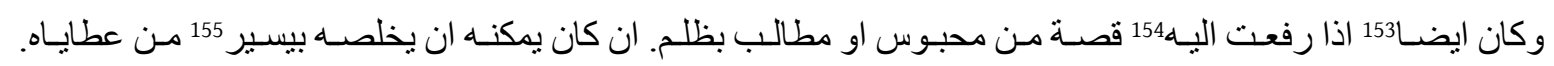

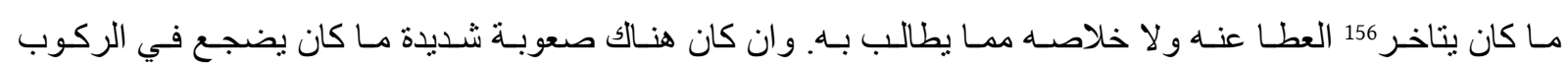

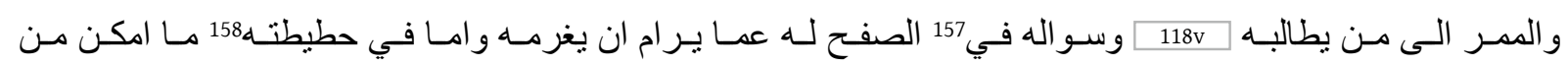

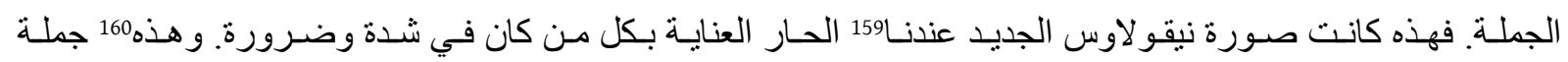
مما يستدل161 به على ما كان يفعله بنفسه وييذل معروفه.

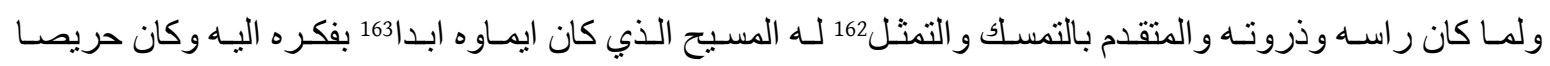

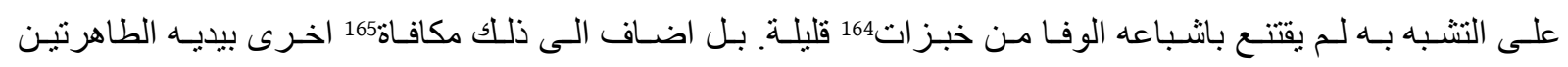

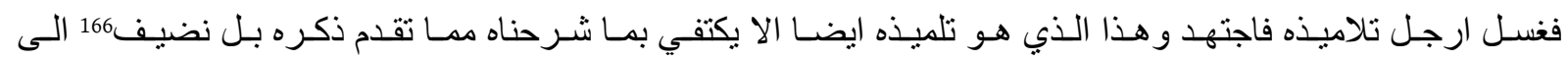

$$
\begin{aligned}
& \text { 147. اليسر: س؛ البشر: ز }
\end{aligned}
$$

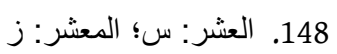

$$
\begin{aligned}
& \text { 149. المسايلين: س؛ المساكين: ز ز ز }
\end{aligned}
$$

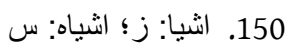

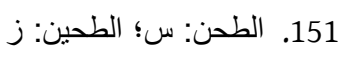

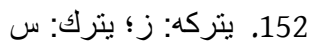

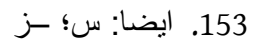

$$
\begin{aligned}
& \text { 154. اليه: س؛ اليه ايضا: ز ز ز }
\end{aligned}
$$

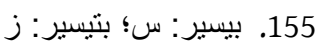

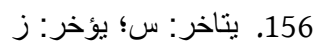

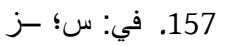

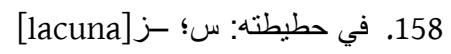

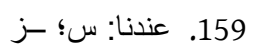

$$
\begin{aligned}
& \text { 160. و هذه: س؛ و هدم: ز سن } \\
& \text { 161. يستدل: س؛ بستبدل: ز ز ز } \\
& \text { 162. بالتمسك والتمثل: س؛ بـ بالتمثل: ز ز }
\end{aligned}
$$

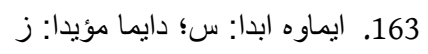

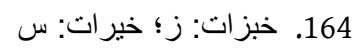

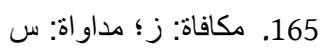

$$
\begin{aligned}
& \text { 166. نضيف: س؛ يضيف: ز ز }
\end{aligned}
$$




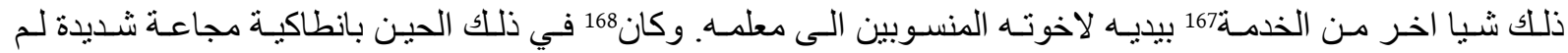

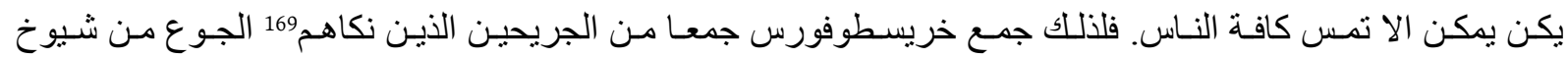

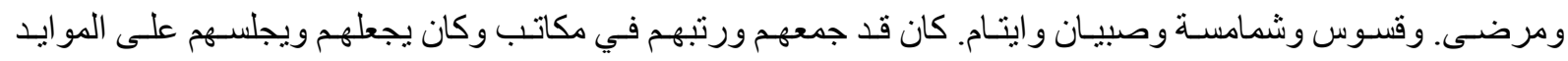

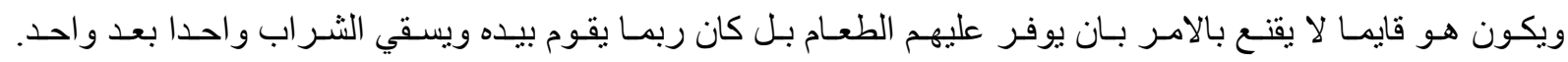

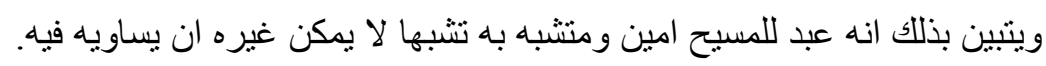

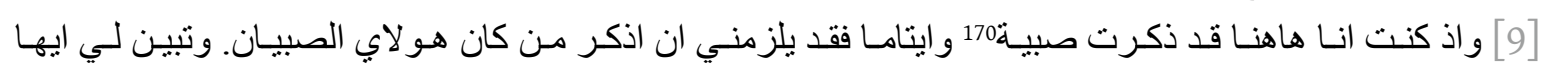

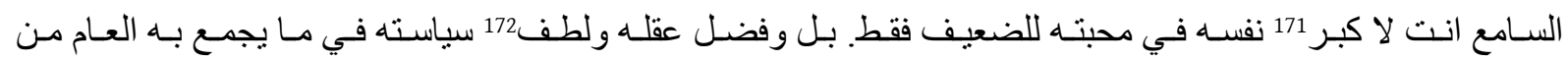

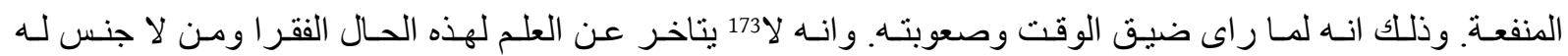

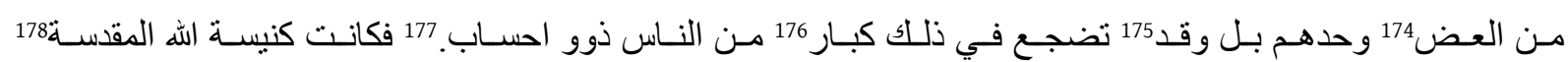

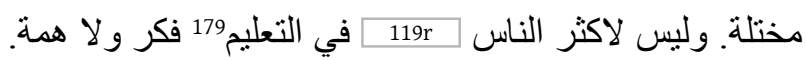

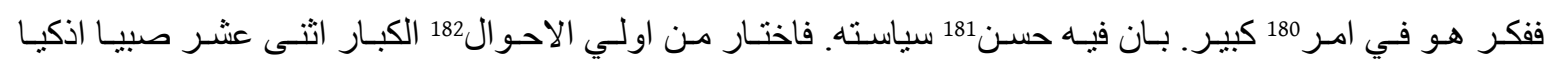

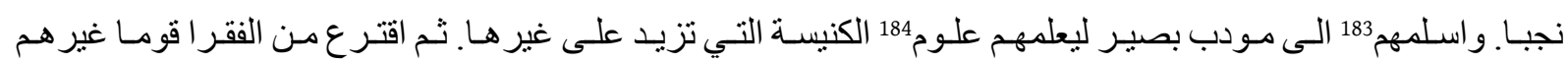

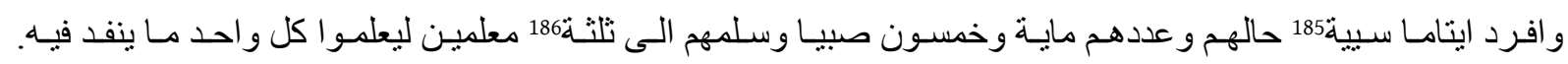

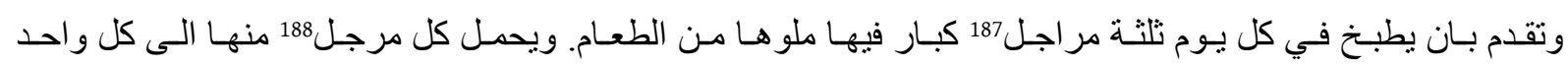

$$
\begin{aligned}
& \text { 167. الخدمة: س؛ المدنية: ز ز }
\end{aligned}
$$

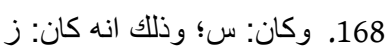

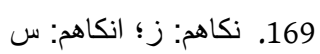

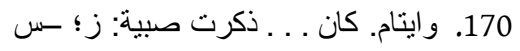

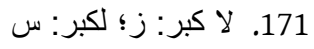

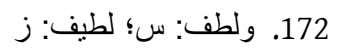

$$
\begin{aligned}
& \text { 173. لا: س؛ لا يتيه ولا: زل زل زل }
\end{aligned}
$$

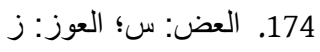

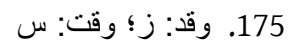

$$
\begin{aligned}
& \text { 176. كبار: س؛ الكبار: ز ز ز } \\
& \text { 177. احساب: س؛ الاحساب: ز ز }
\end{aligned}
$$

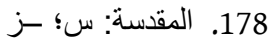

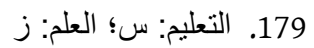

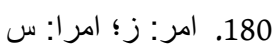

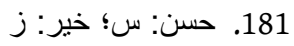

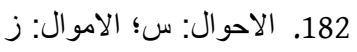

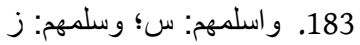

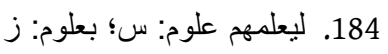

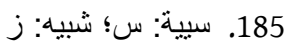

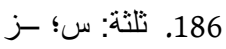

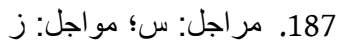

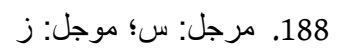

Al- 'Ușūr al-Wusțā 29 (2021) 


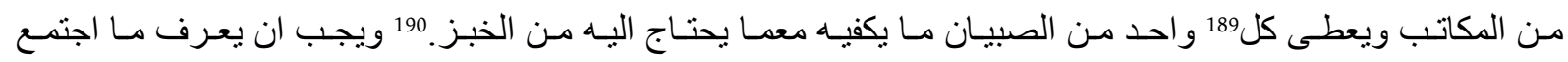

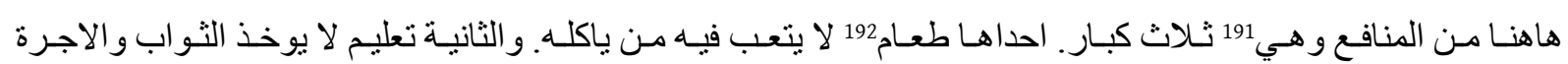

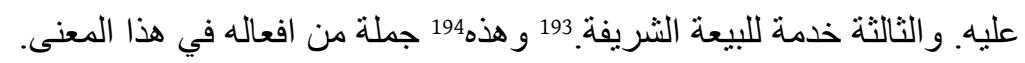

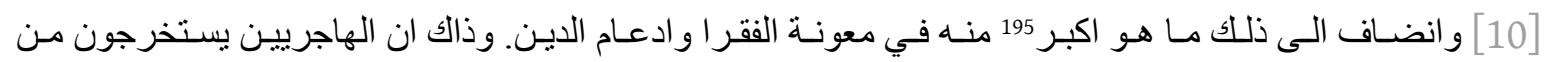

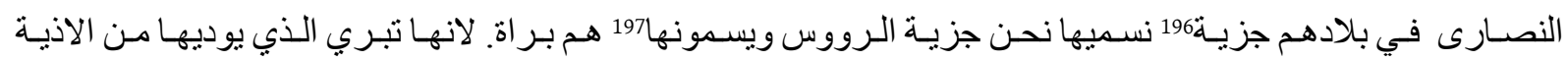

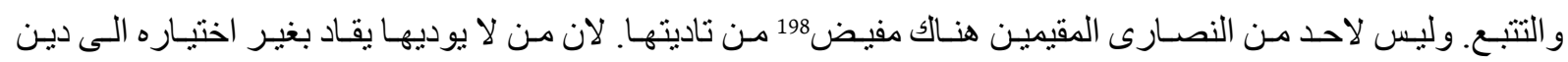

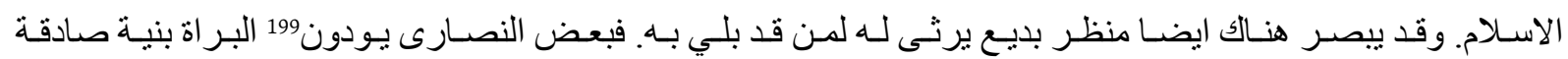

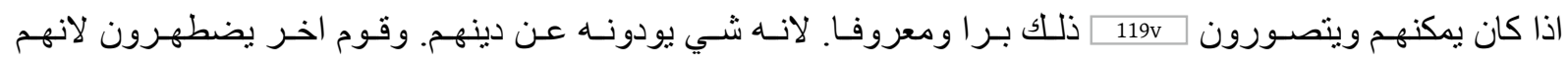

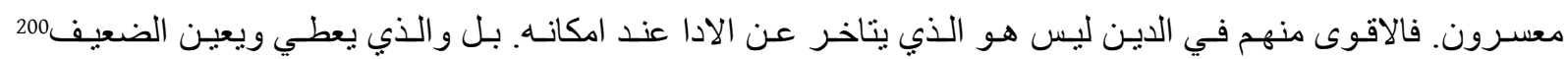
فيما بيتادى منه.

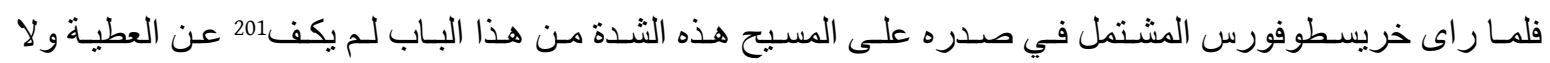

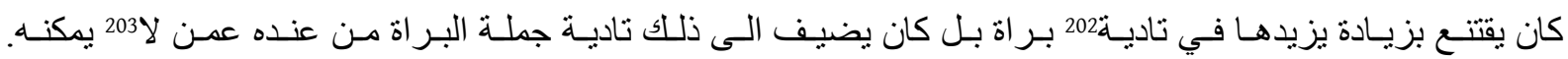

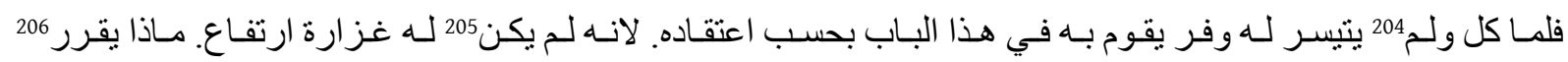

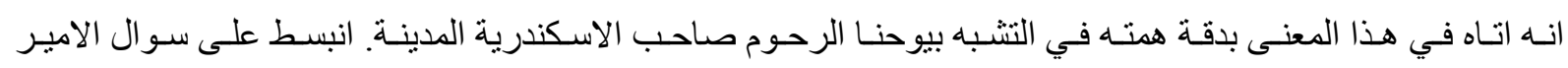

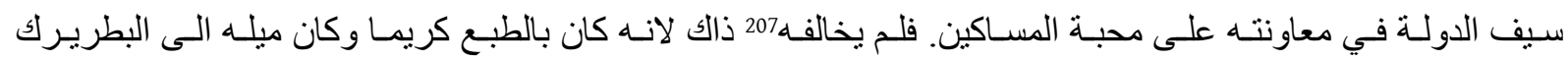

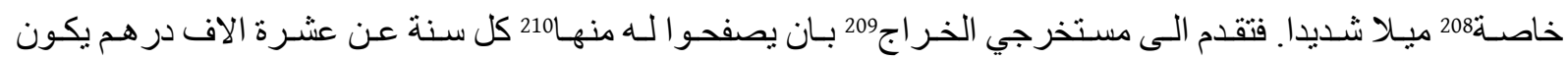

$$
\begin{aligned}
& \text { 189 - 189. كل: س؛ لكل: ز ز }
\end{aligned}
$$

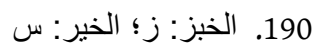

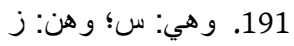

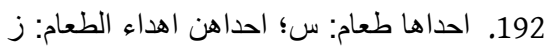

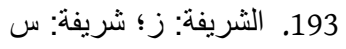

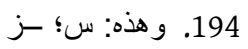

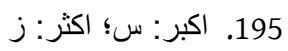

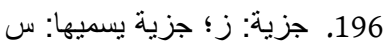

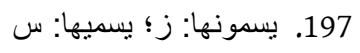

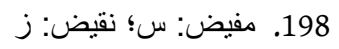

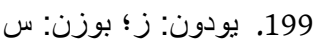

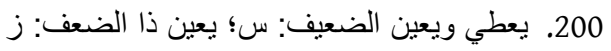

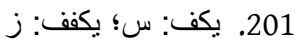

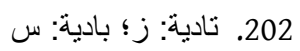

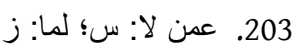

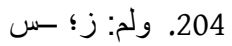

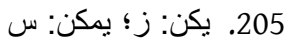

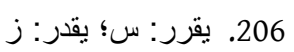

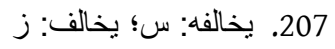

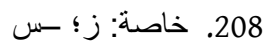

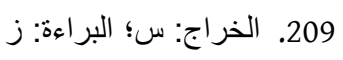

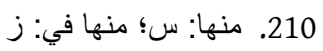

Al-'Ușūr al-Wusțā 29 (2021) 


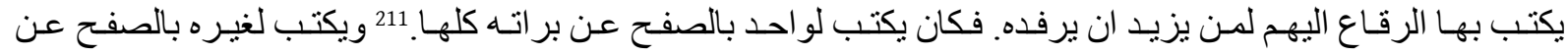

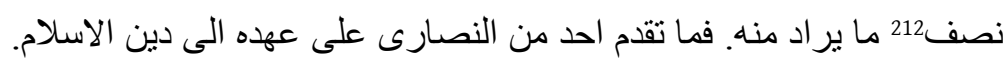

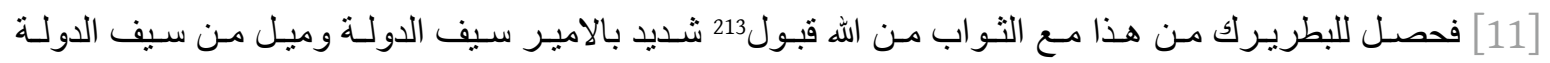

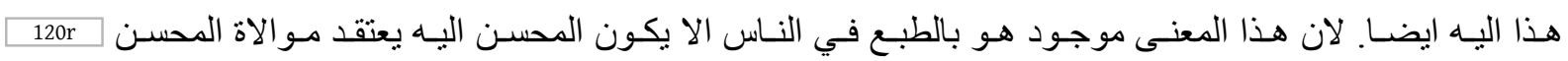

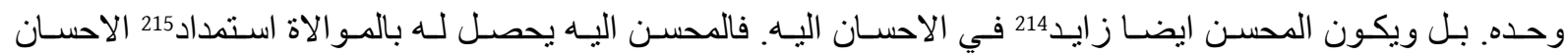

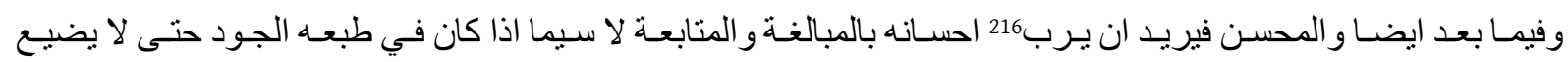
ما تقدم من احسانه بالامساك.

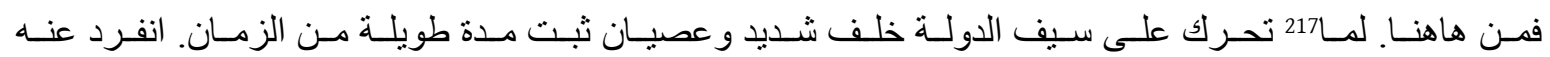

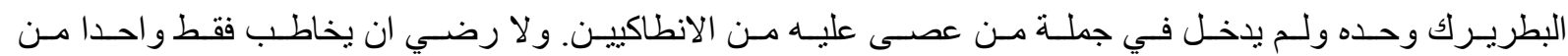
المتمردين. وكان السبب في هذا العصيـان ان الملك نقفور المغبوط لمـا ملك بلـد الثغر الاكثر منـه عنـوة وبحرب وطرسوس

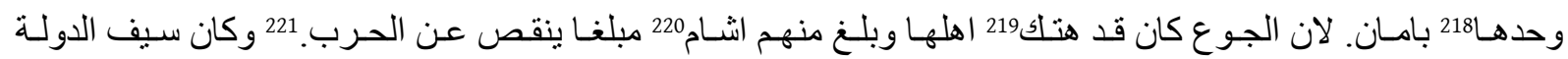

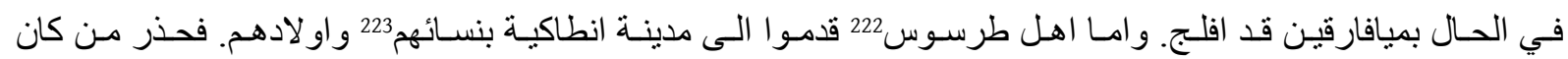

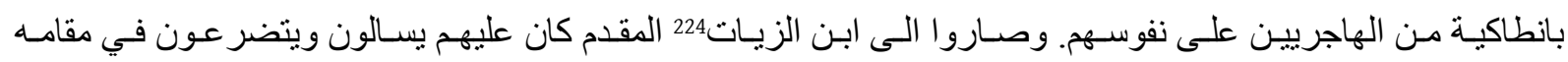

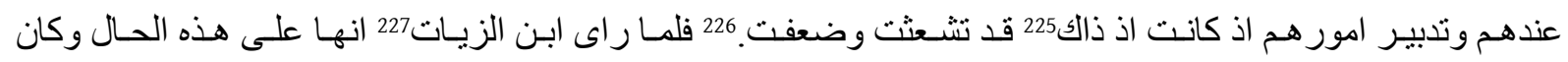
جز عهد28 من نققور الملك يكره. 229 امتنع من المقام بالكلية.

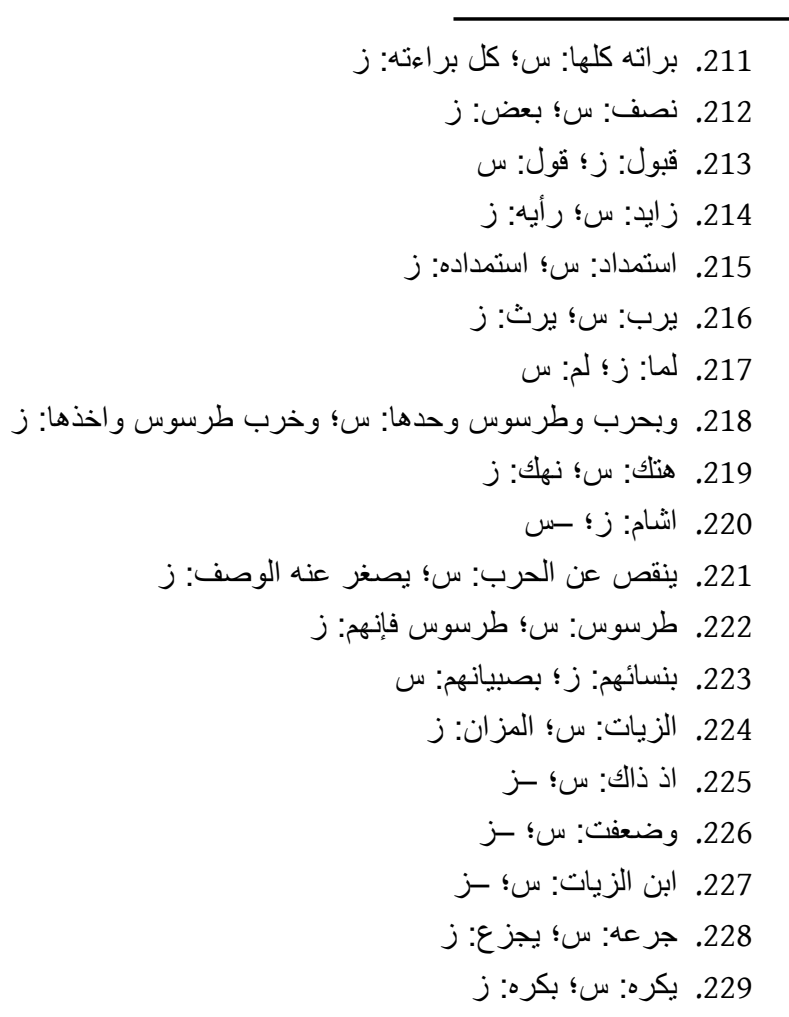

Al- 'Ușūr al-Wusțā 29 (2021) 


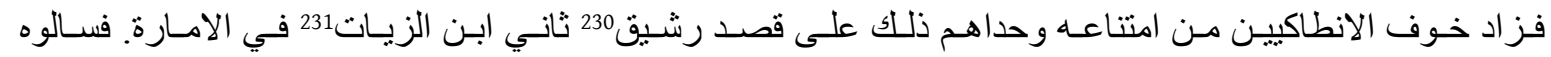

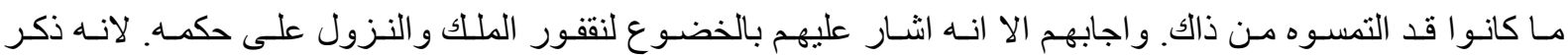

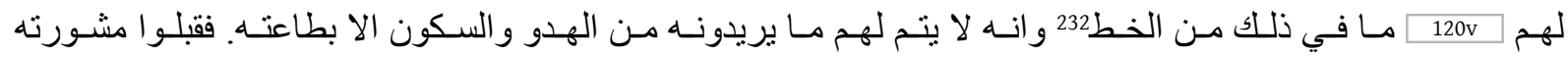

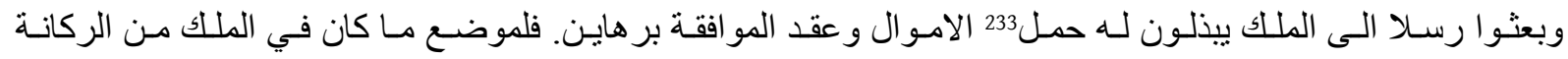

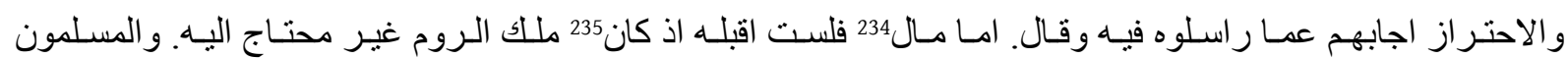

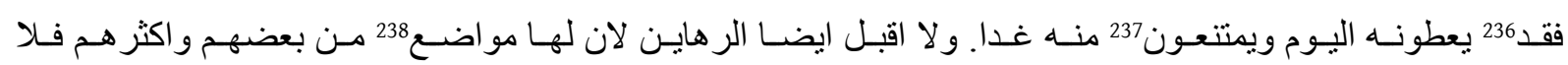

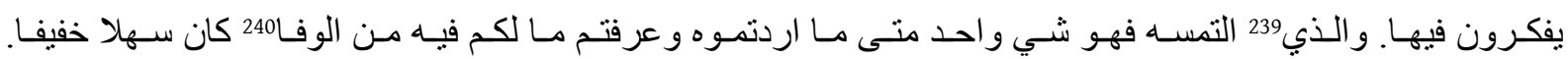

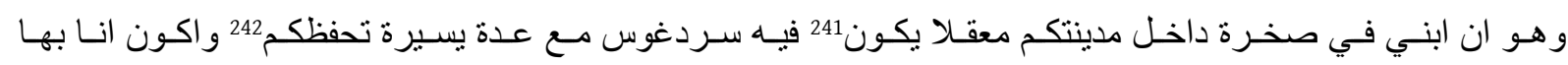

\section{مستظهر ا.}

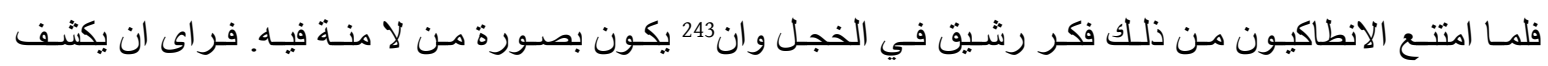

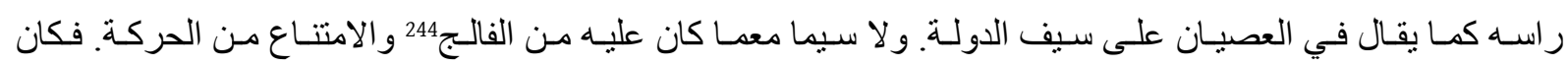

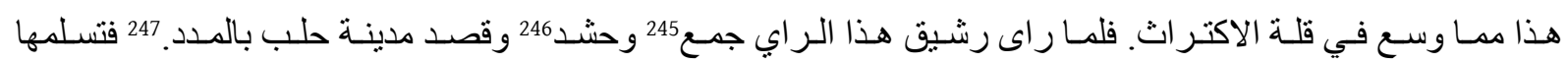

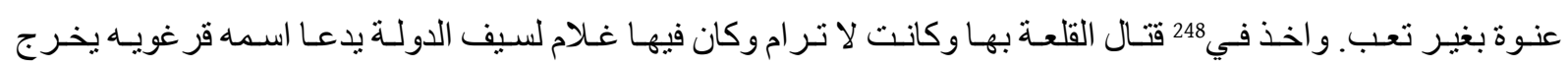

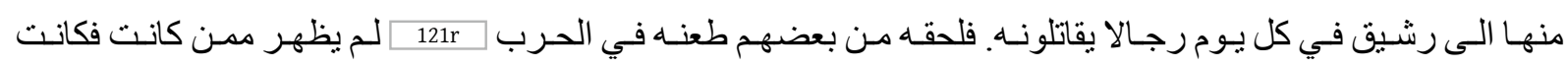

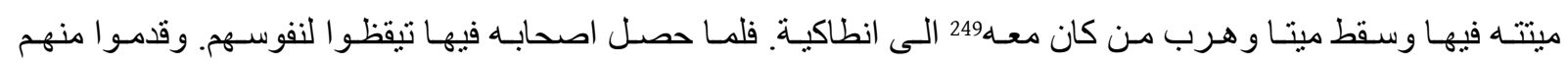

$$
\begin{aligned}
& \text { 230. رشيق: س؛ رشيق النسيمي الوارد من طرسوس وكان: ز }
\end{aligned}
$$

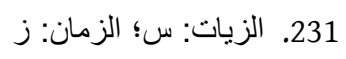

$$
\begin{aligned}
& \text { 232. الخط: س؛ الخطأ: ز ز }
\end{aligned}
$$

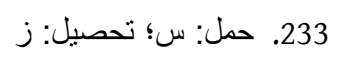

$$
\begin{aligned}
& \text { 234. مال: س؛ المال: ز ز } \\
& \text { 235. اذ كان: س؛ لان: زل: زل ز } \\
& \text { 236. فقد: ز؛ فقد فقد: سل سل سن }
\end{aligned}
$$

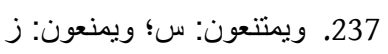

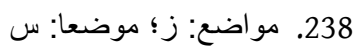

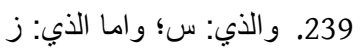

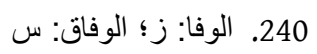

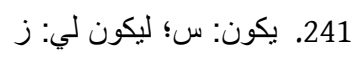

$$
\begin{aligned}
& \text { 242. تحفظك: س؛ تحفظهم: ز ز } \\
& \text { 243. وان: س؛ و وانه: ز ز }
\end{aligned}
$$

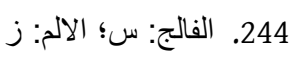

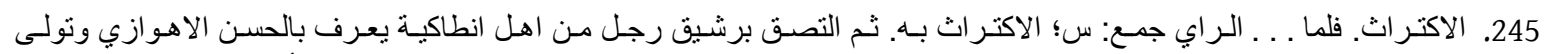

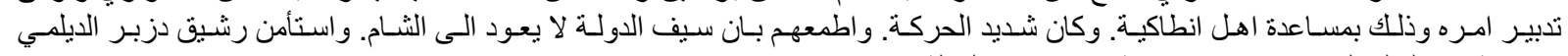

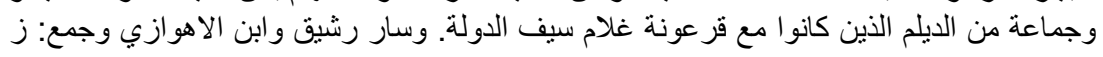

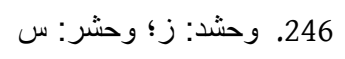

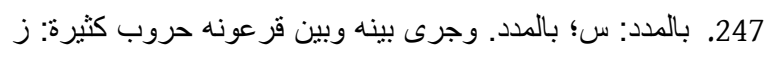

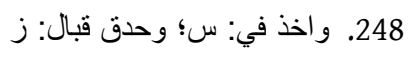

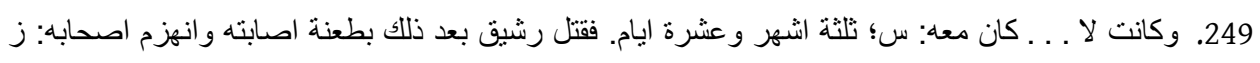




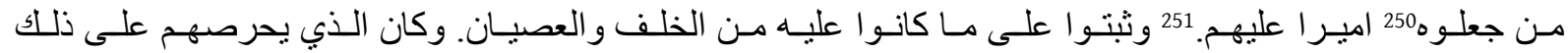

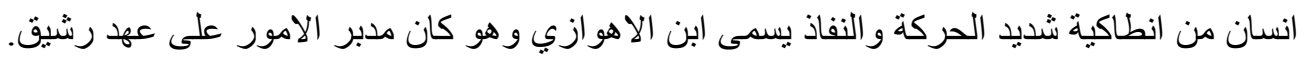

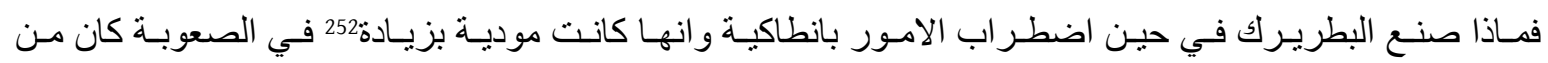

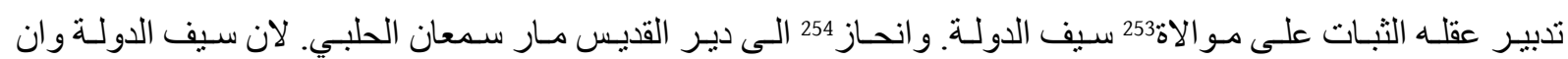

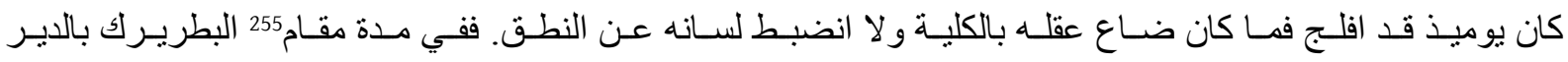

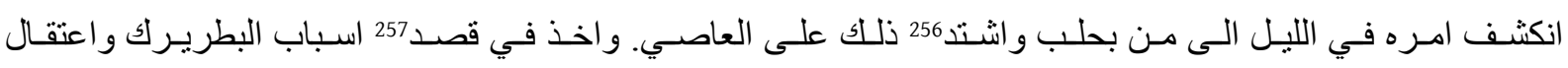

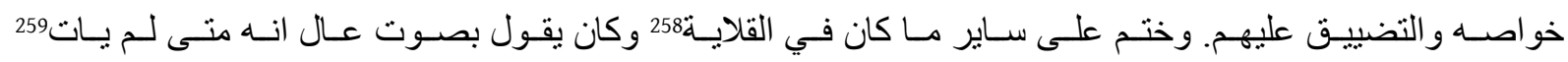
البطريرك ويتلافاه260 و الا خرج معله الى ولى زيادة فيما يغمه.

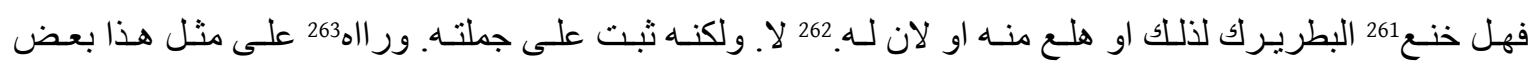

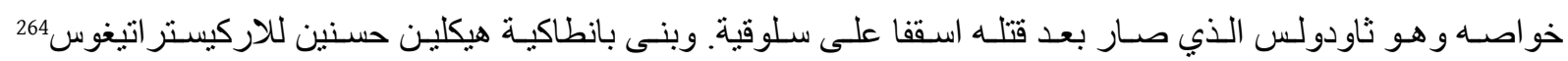

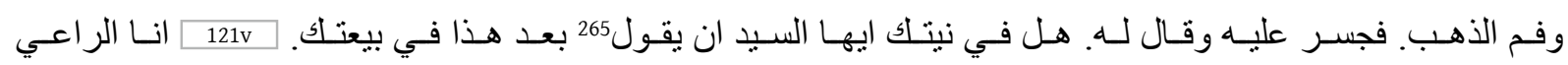

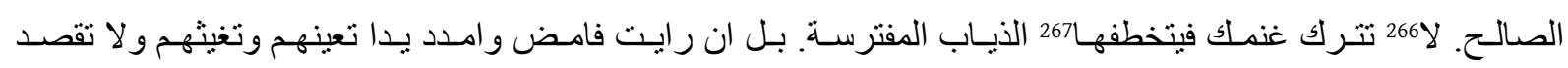

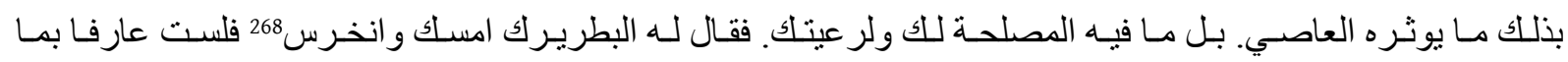
تقوله. فلزمه الامساك.

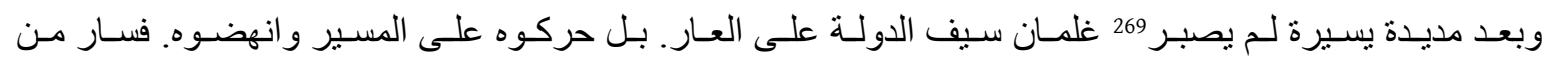

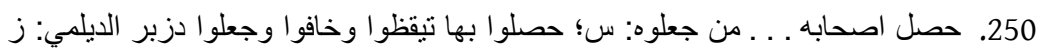
251. عليهم: س؛ عليهم وابن الاهوازي المدبر له: ز ز

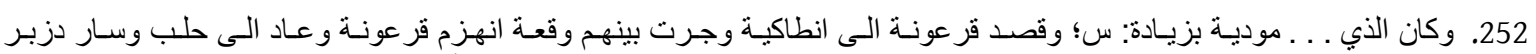

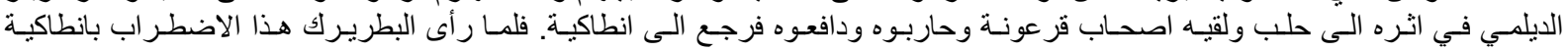

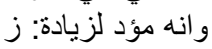

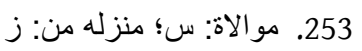

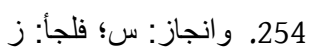

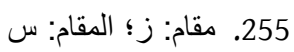

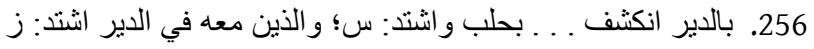

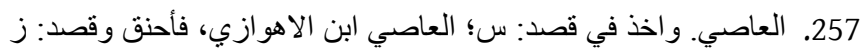

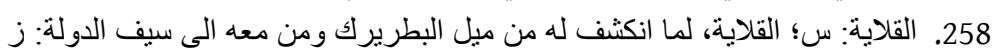

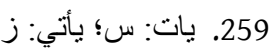

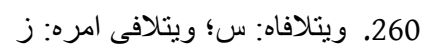

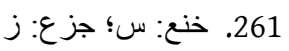

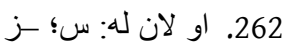

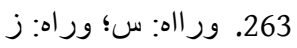

264. للاركيستر اتيغوس: صححته؛ للاكسير اتيقوس: س؛ الازكسعوطس: ز

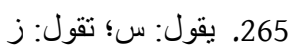

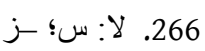

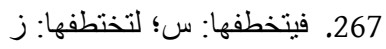

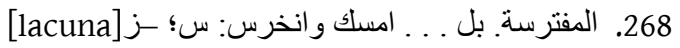

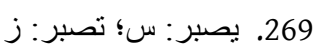

Al- 'Ușūr al-Wusțā 29 (2021) 


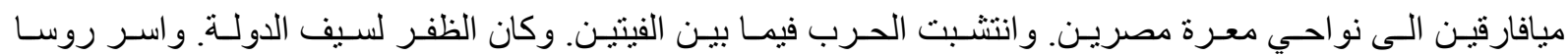
عسكر الخوارج وحصلهم 270 في القيود و السلاسل.

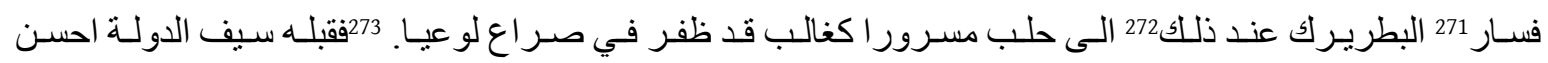

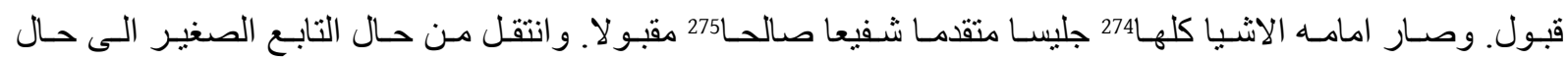

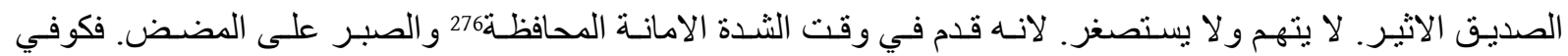

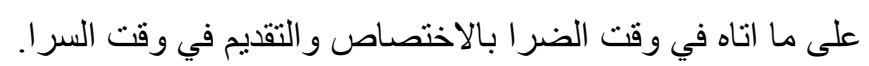

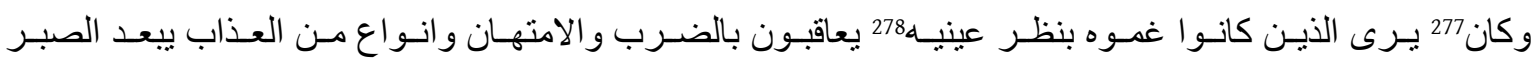

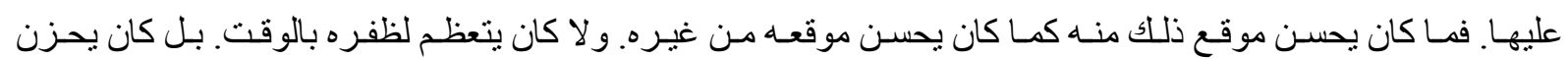

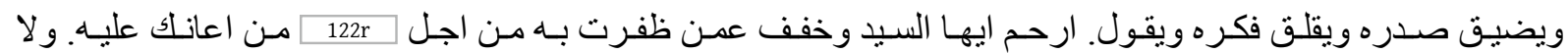

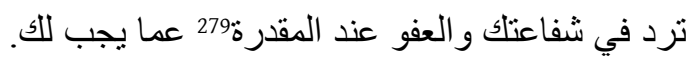

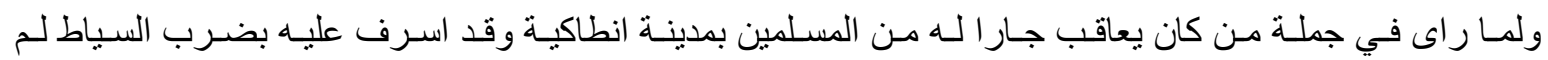

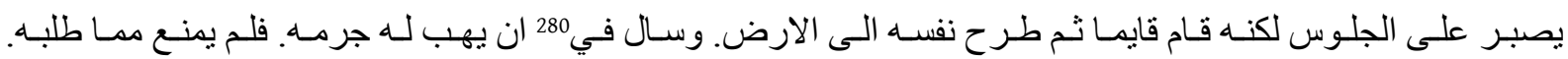

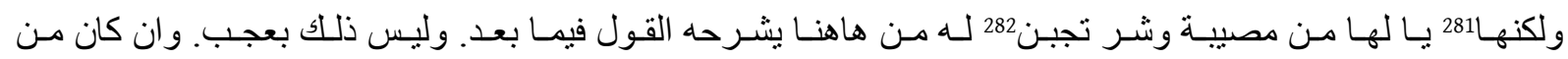

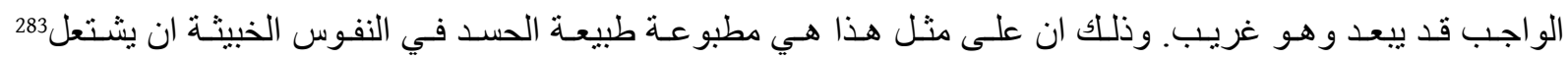

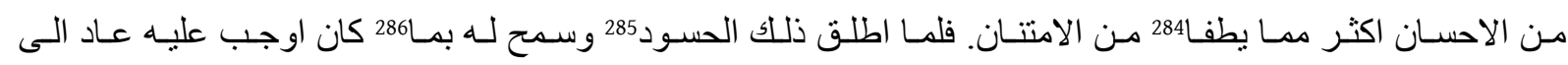
انطاكية بريا من التبعة. 287 وليت ذلك لم يكن. 288

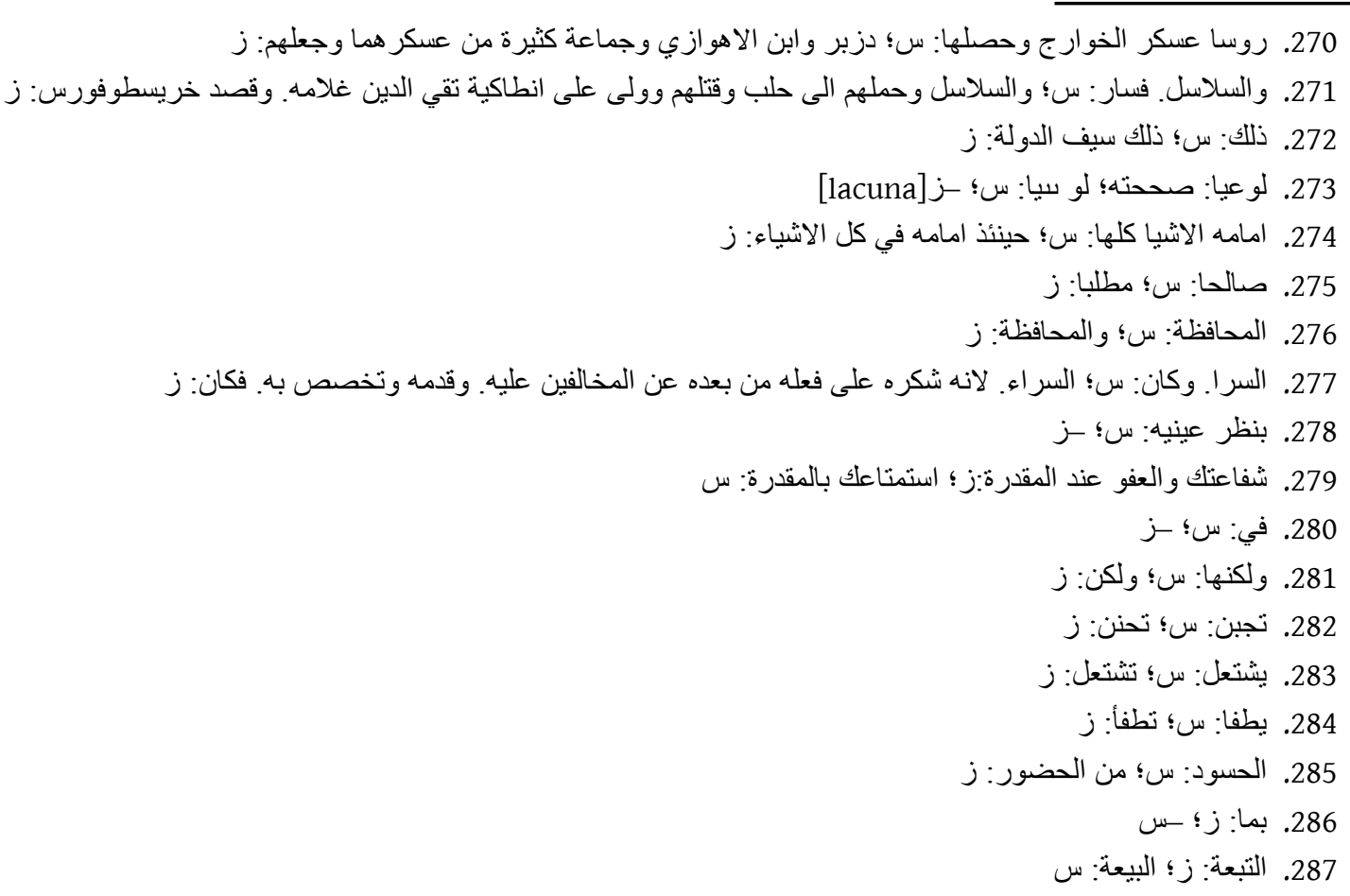

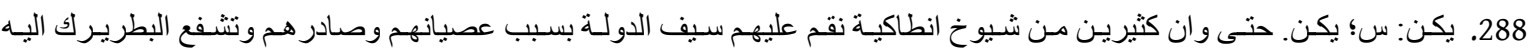

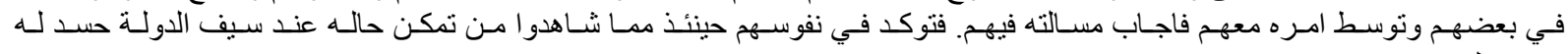
وحقد عليه: ز فيمتر 


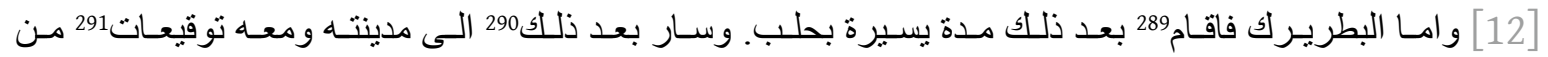

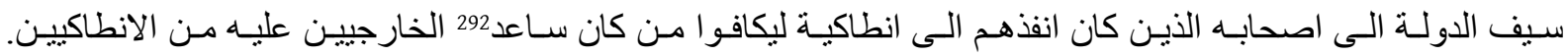

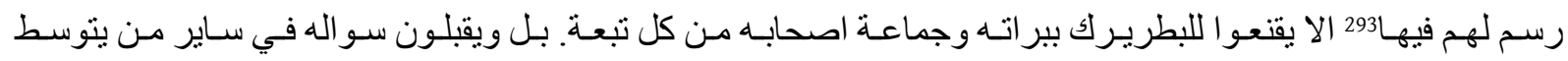
امره بمقدار ما يمكن القبول منه. لان مثل هذا كان غرض

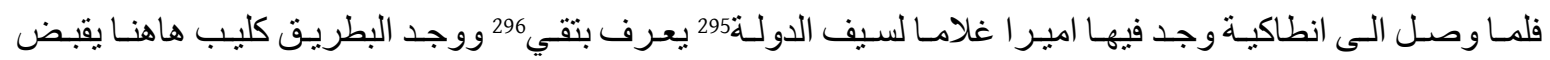

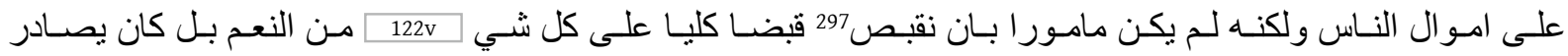

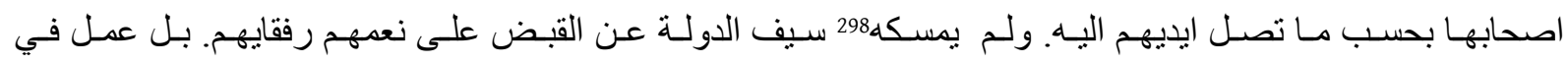

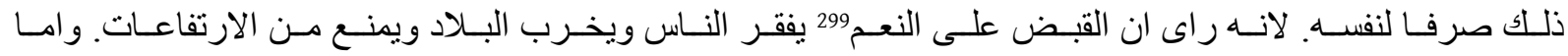

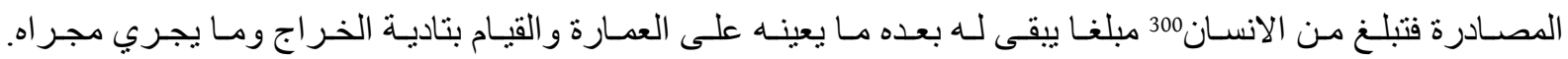

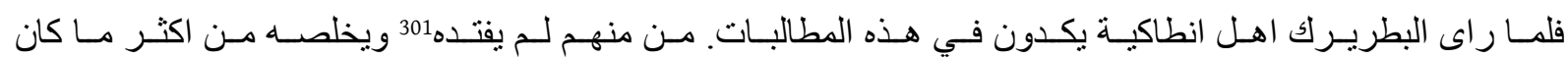

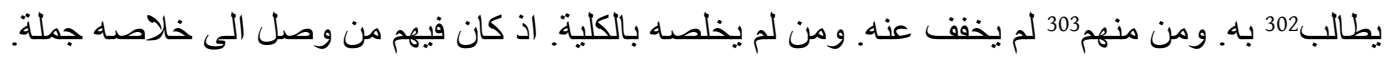

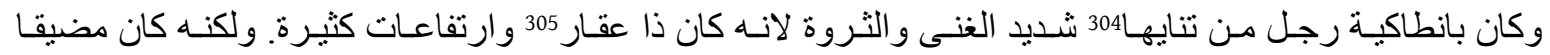

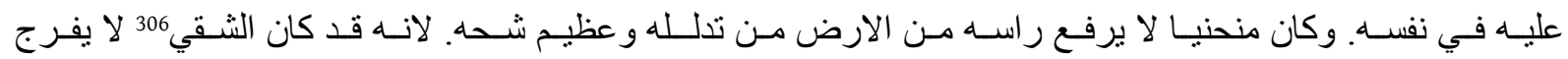

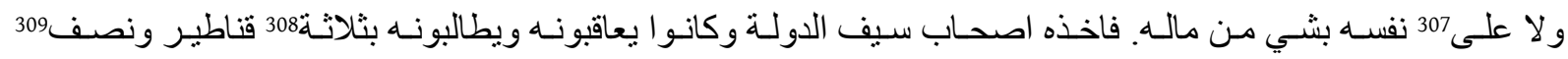

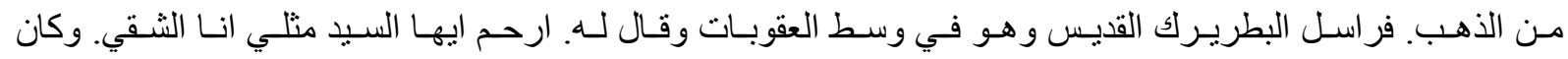

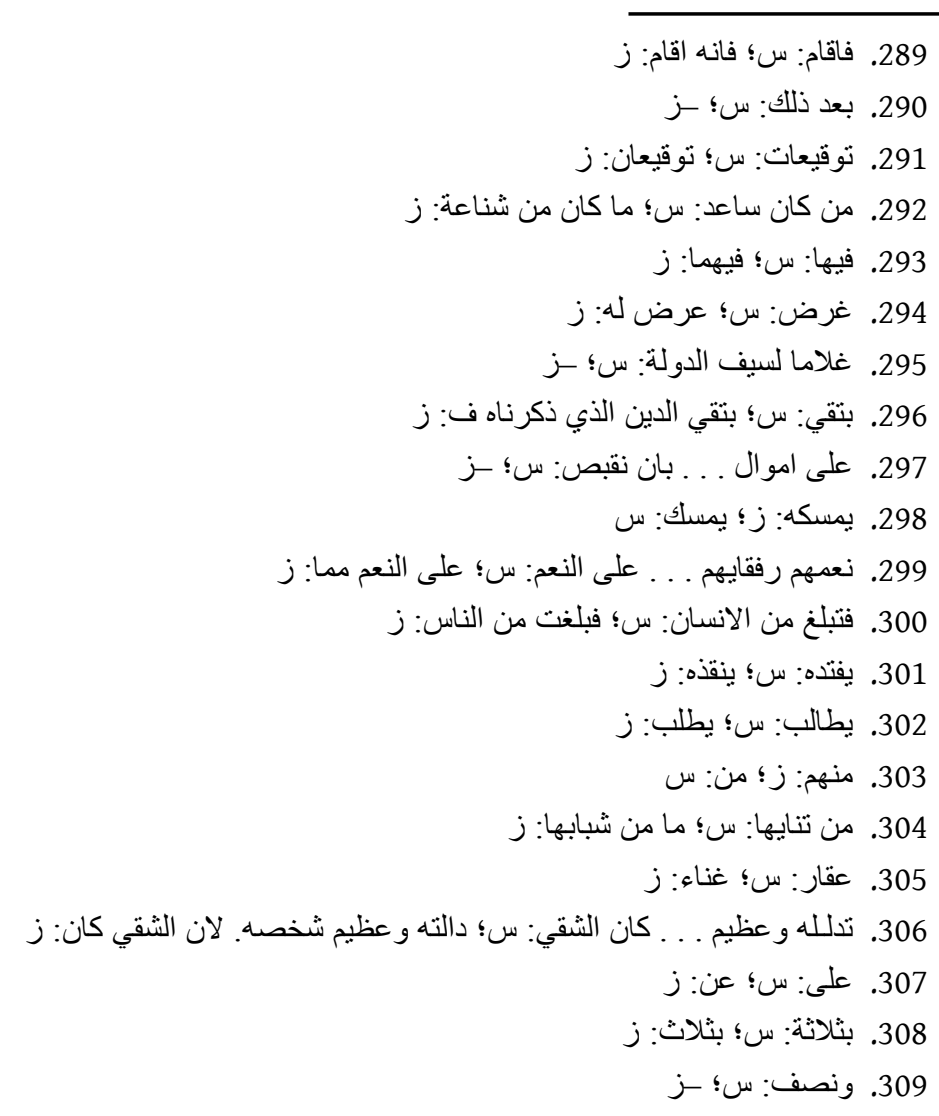

Al- 'Ușūr al-Wusțā 29 (2021) 


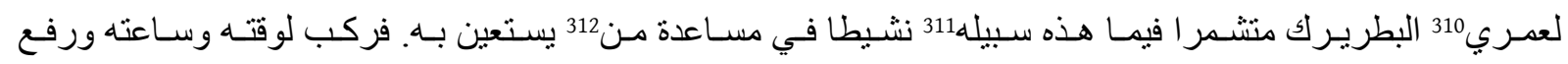

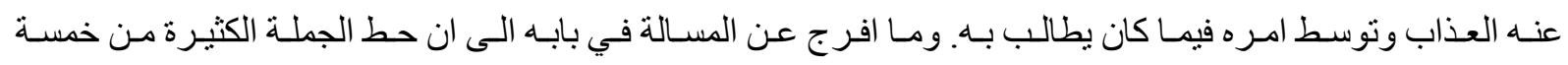

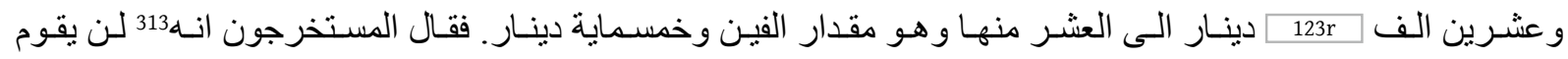

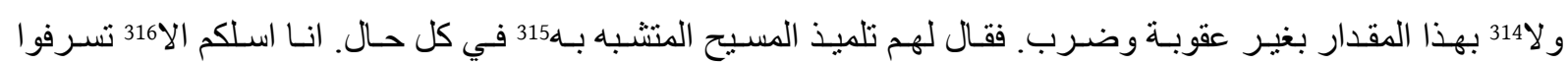

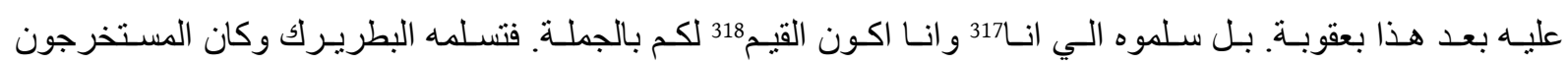

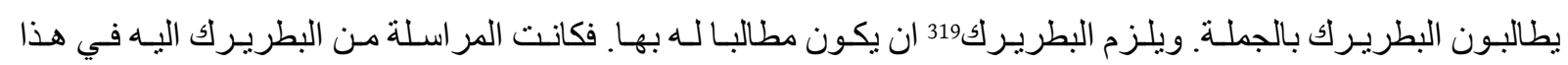

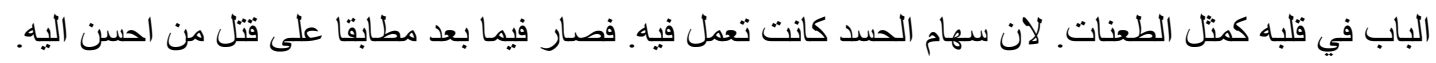

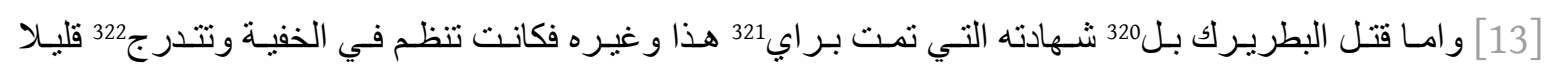

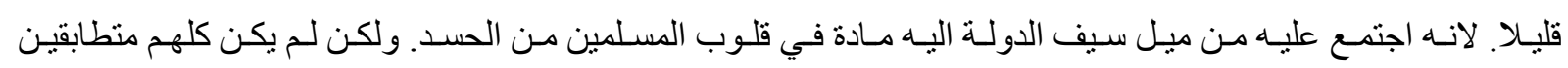

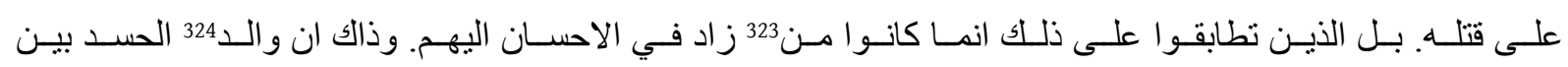
فيهم فعله. 325

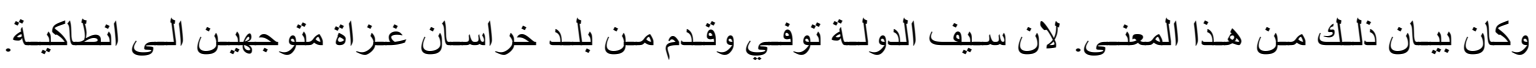

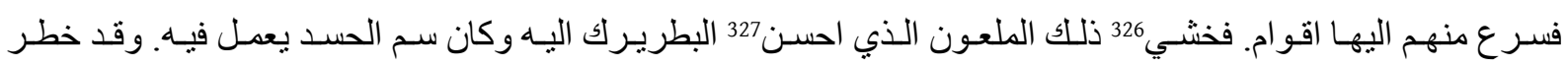
له ان يدبر عليه328 من تمكن البطريرك فيما بعد. اذا مات329 وصّل صاحب الخر اسانية330 الا يتمكن منه فيفوته قتله.

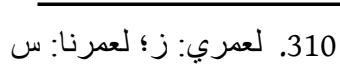

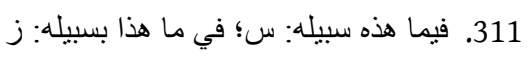

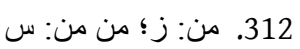

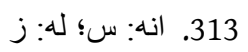

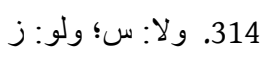

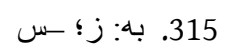

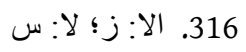

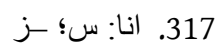

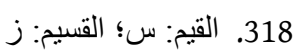

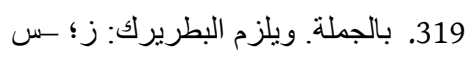

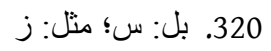

321. براي: س؛ من: زن زن

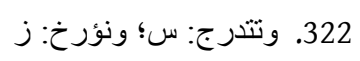

323. من: س؛ ممن: ز ز

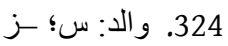

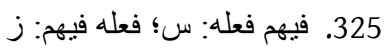

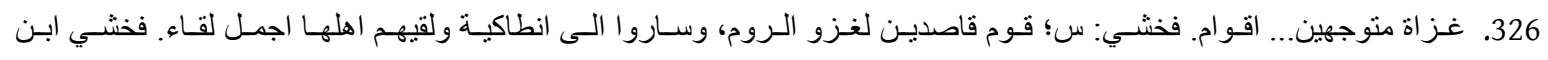




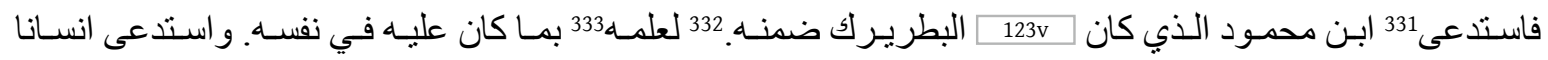

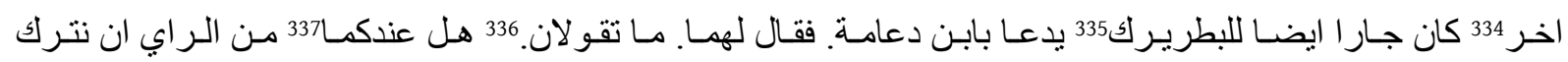

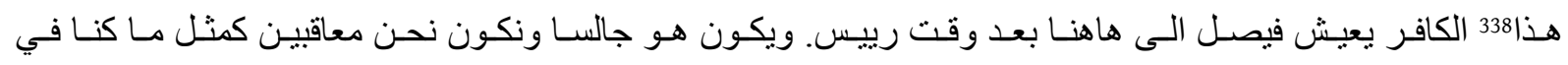

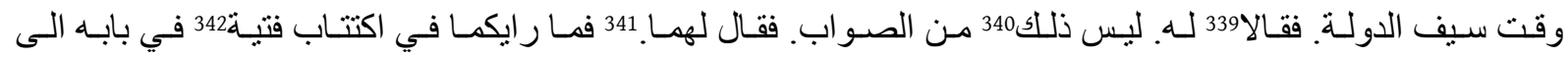

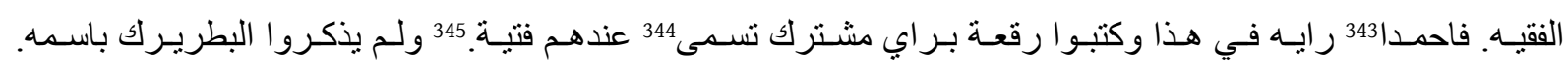

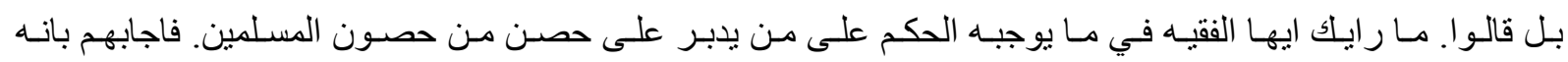
يجب عليه القتل.

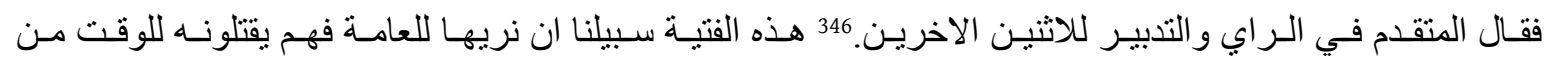

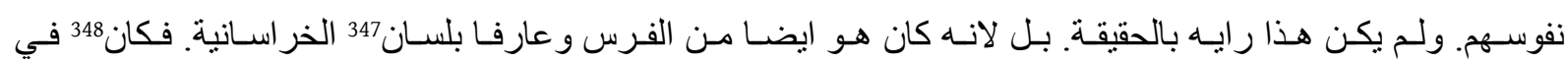

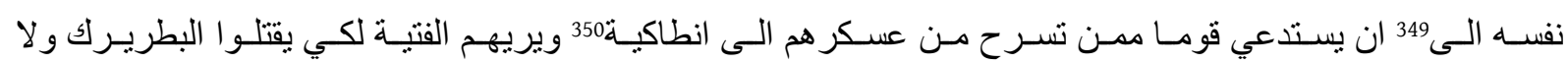
يجنحو إ351 في ذلك. فكان هذا مما اقنعهم وحداهم على مانى ما ار اده.

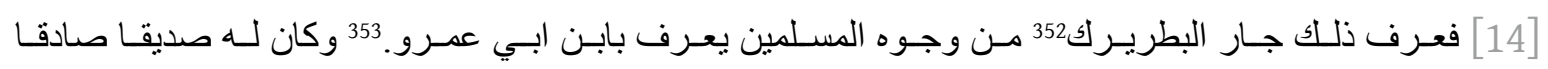

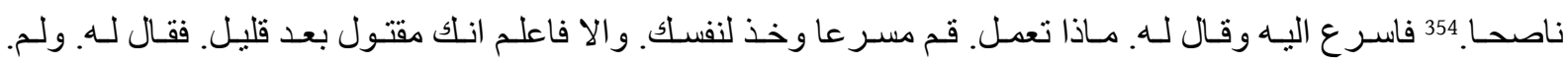

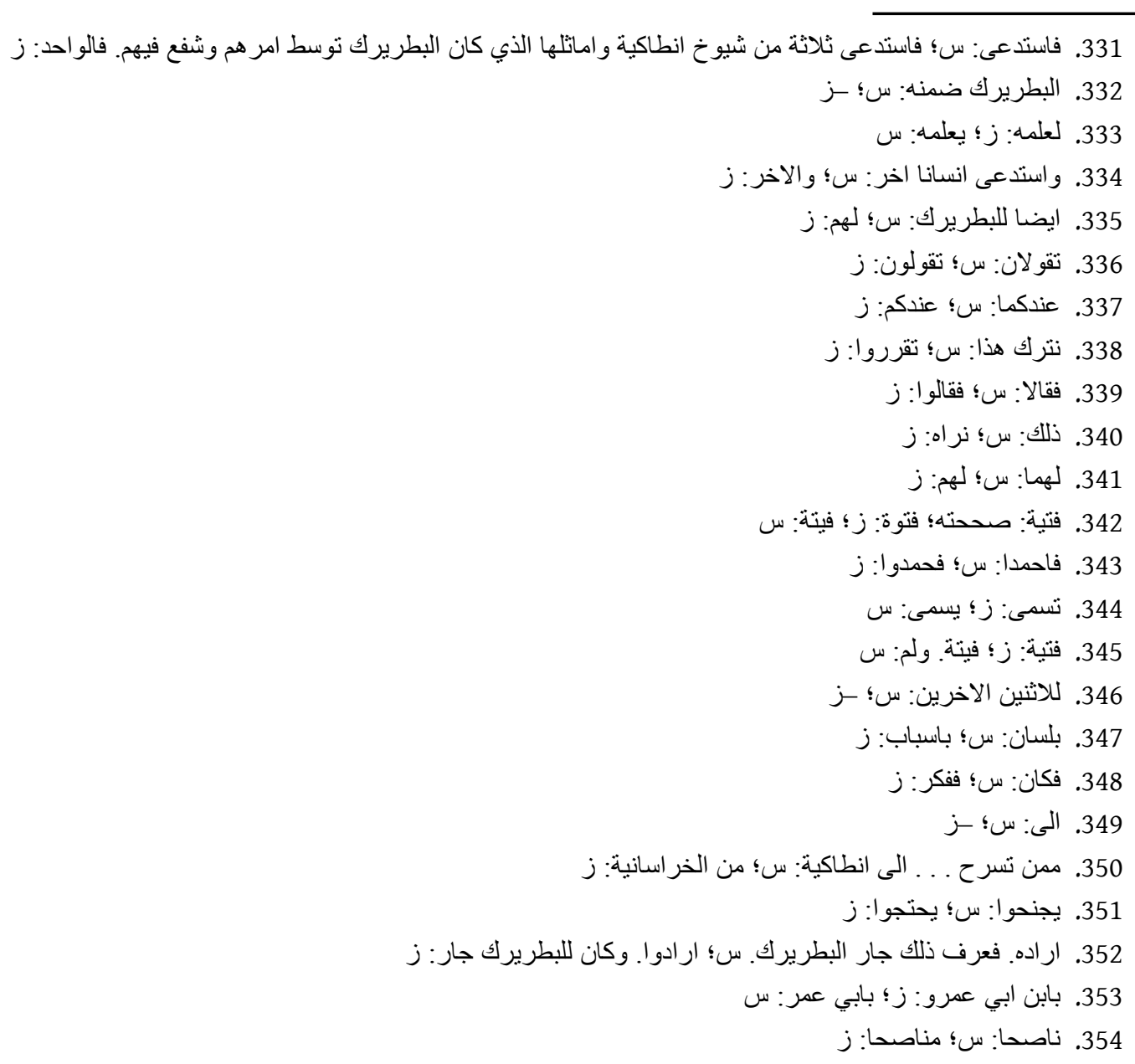

Al-'Ușūr al-Wusțā 29 (2021) 


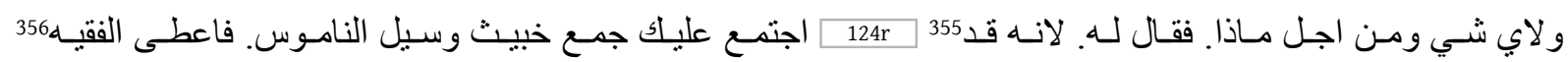

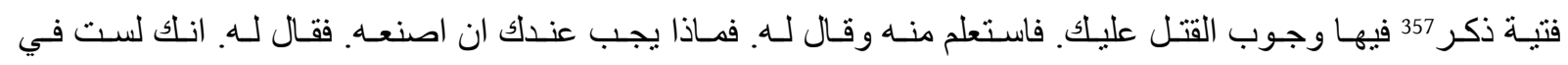

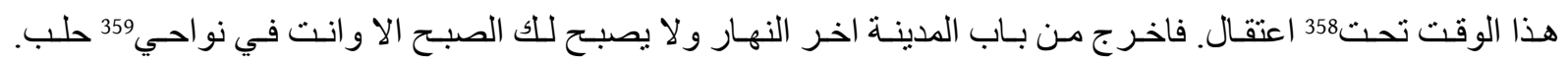

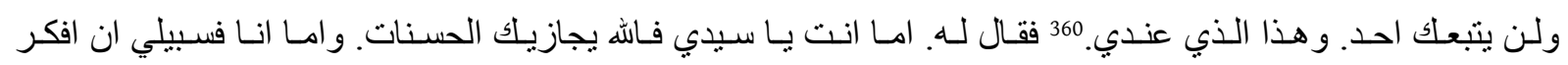
فيما اعمله.

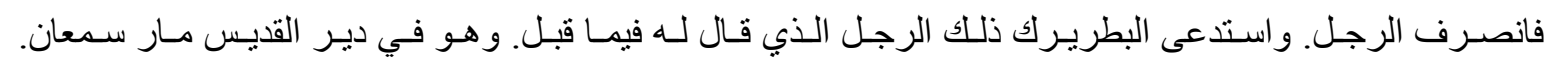

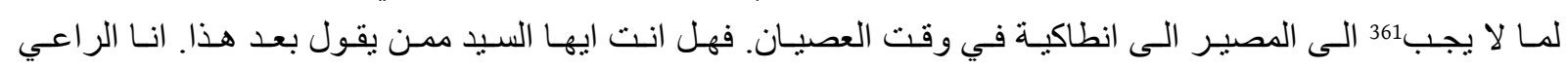

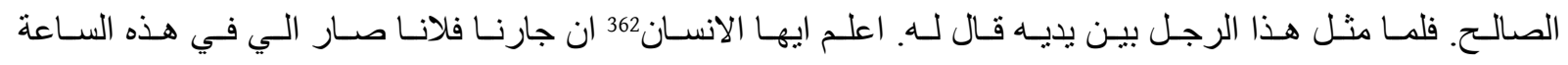

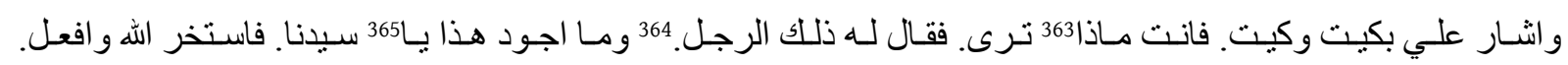

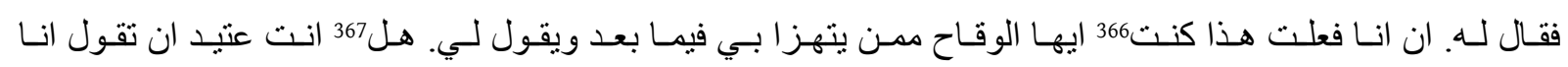

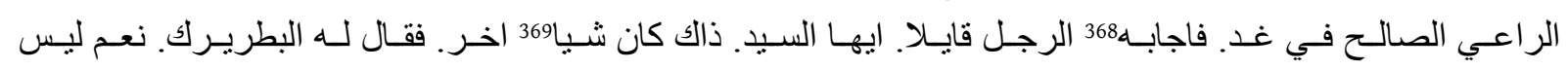

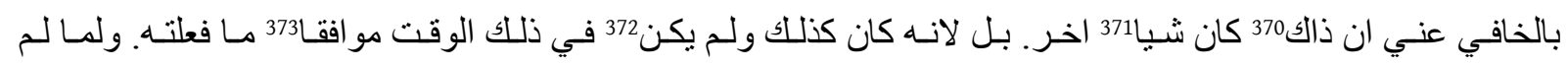

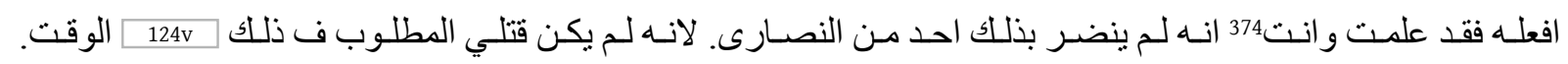

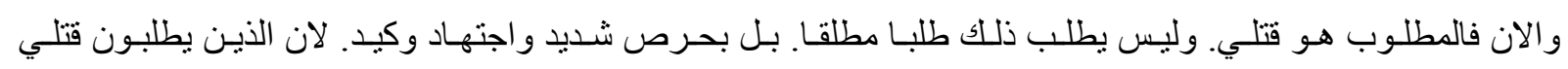

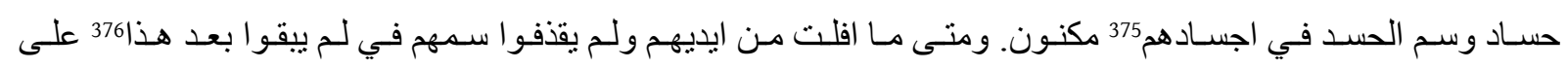

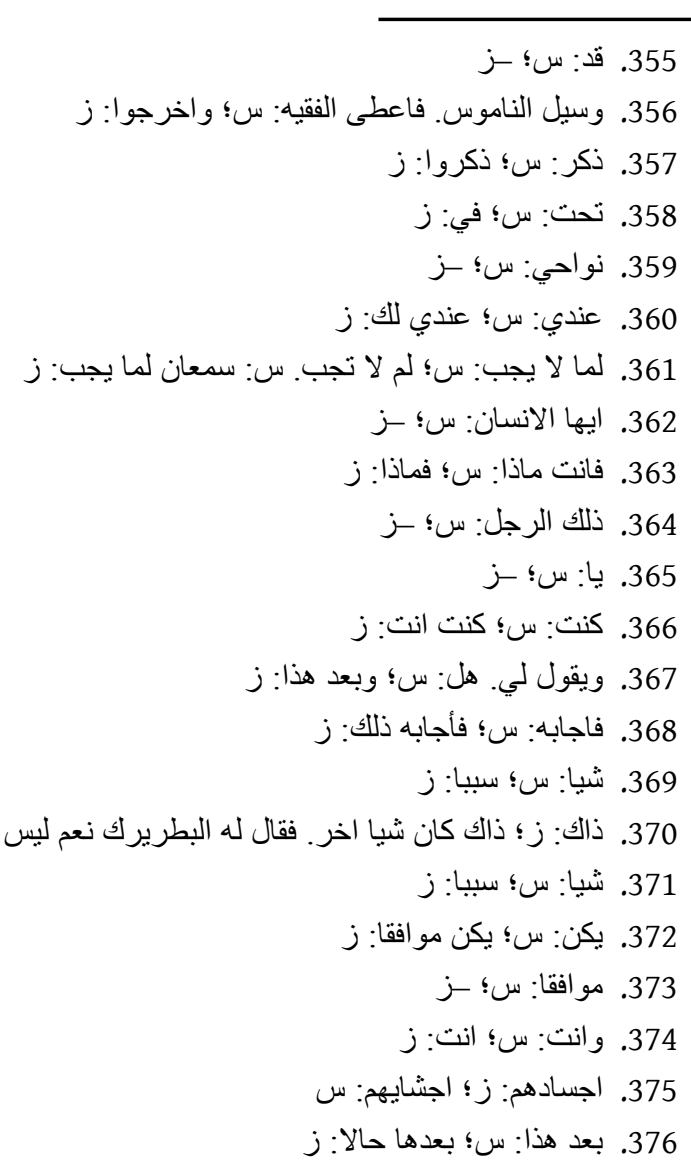

Al-`Ușūr al-Wusțā 29 (2021) 
نصر انـي و لا على كنيسـة. ولكن هذا الوقت377 الذي يجب 378 لـي379 ان اقول380 يـا هذا. ليس انـا الر اعي الصـالـح فقط.

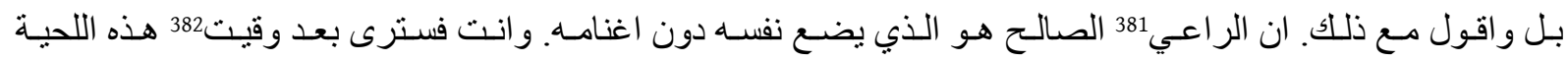

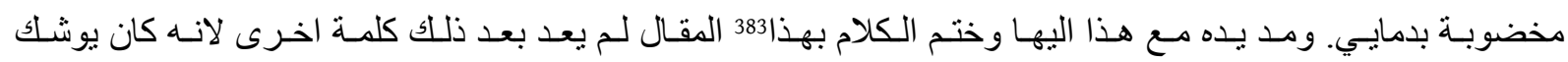
انه قد تشور 384 من الفكر.

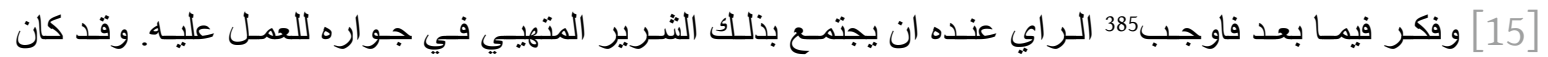

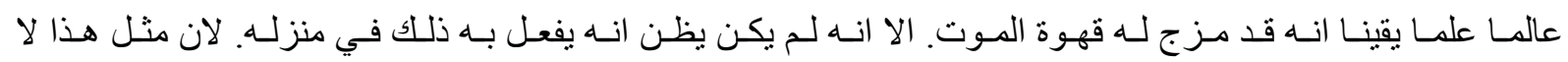

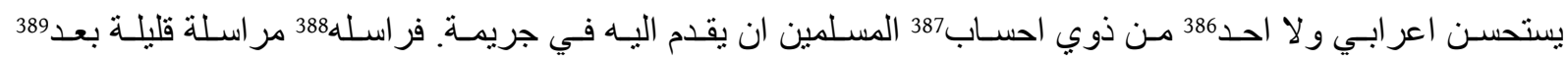

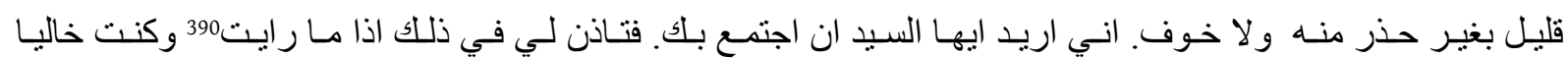

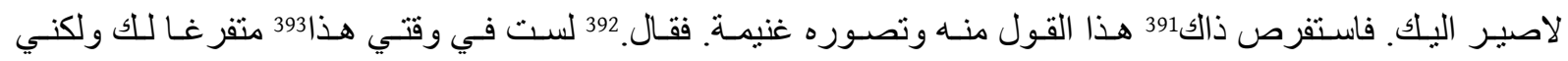

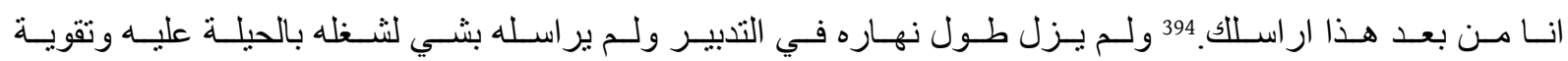
ما يطبخه له.

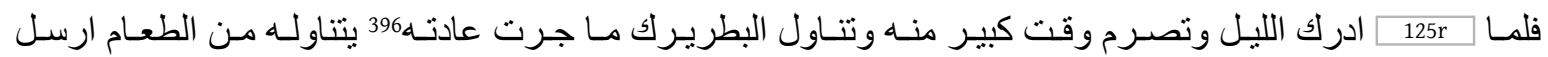

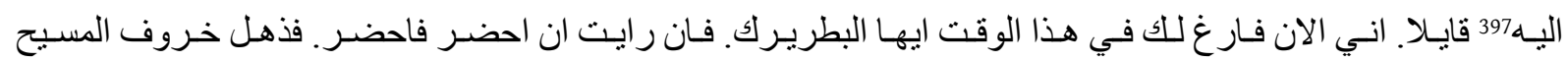

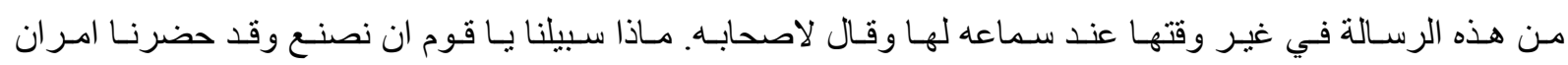

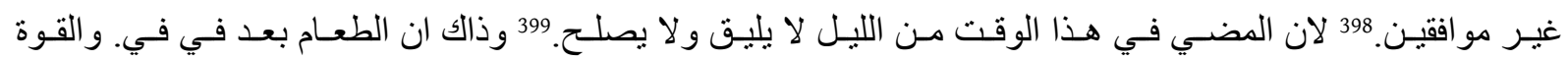

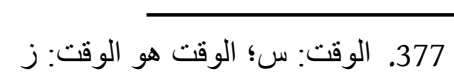

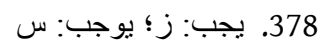

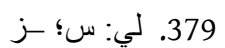

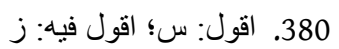

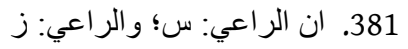

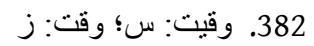

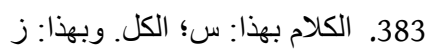

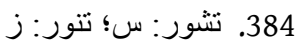

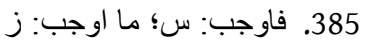

$$
\begin{aligned}
& \text { 386. اعرابي و لا احد: س؛ انس ان يكون إلا لاحد: ز }
\end{aligned}
$$

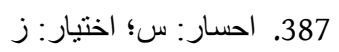

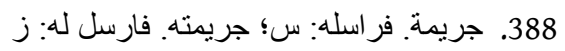

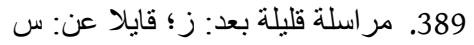

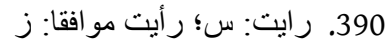

$$
\begin{aligned}
& \text { 391. فاستفرص ذاك: س؛ س؛ فلما سمع ابن مانك ذلك استفرص: ز ز }
\end{aligned}
$$

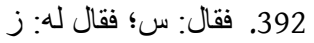

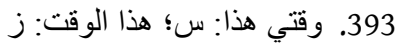

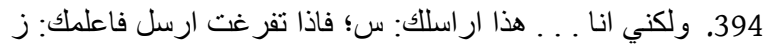

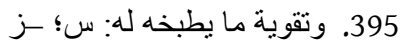

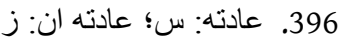

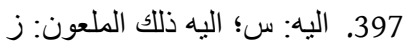

$$
\begin{aligned}
& \text { 398. مو افقين: س؛ مو الفقان: زل زل } \\
& \text { 399. يصلح: س؛ يصلح اذ كان: ز ز زلفئ }
\end{aligned}
$$

Al- 'Ușūr al-Wusțā 29 (2021) 


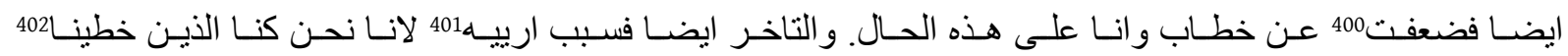

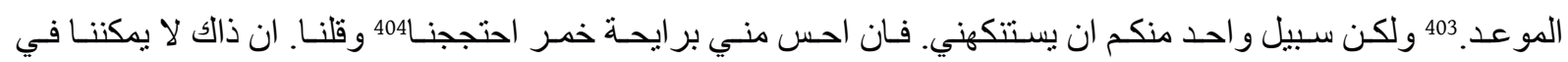

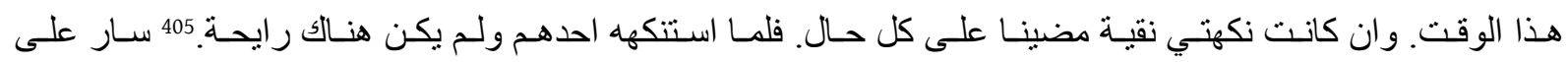
قدميه كمثل حمل 406 باختياره الى الذبح.

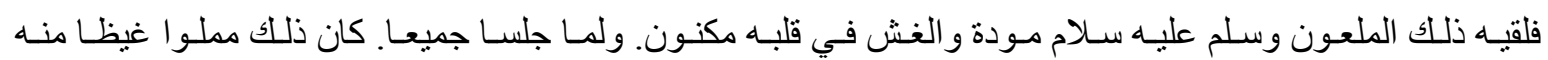

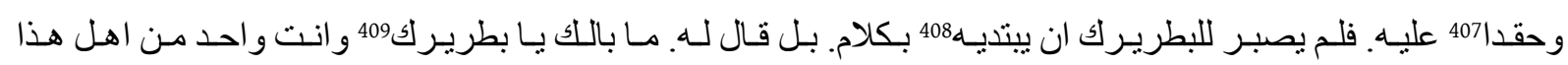

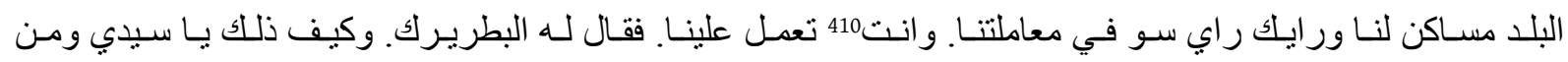

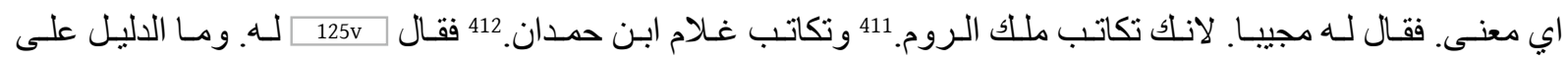

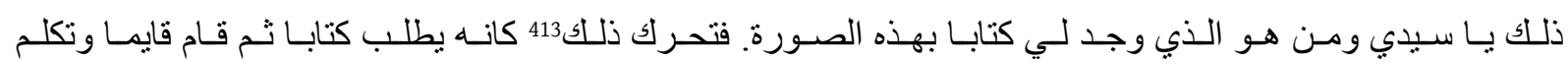
بالفارسية و استدعى لمن كان قـ414 اعدهم 415 للقتل من الخر استانية.

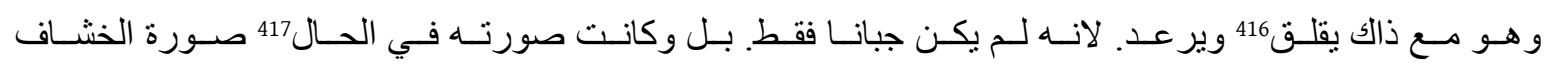

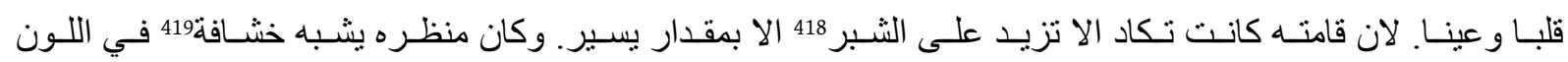

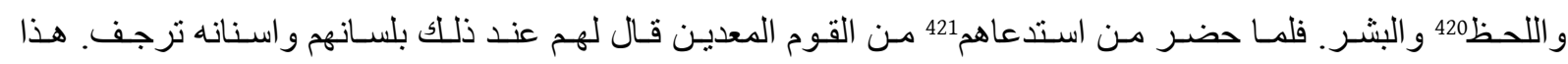
هو المطلوب. هذا هو الذي يريد ان يسلم هذه المدينة. هذا هو عدو المسلمين. فدونكن واياه بضعوه بغير اشفاق.

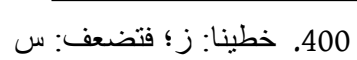

$$
\begin{aligned}
& \text { 401. و التاخر ايضا فسبب اريبه: س؛؛ والتأخير ايضا بسبب بلية: ز }
\end{aligned}
$$

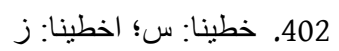

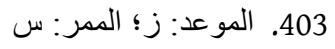

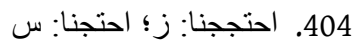

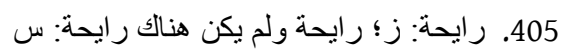

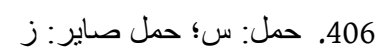

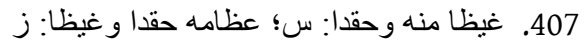

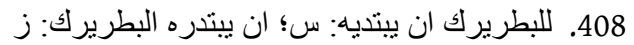

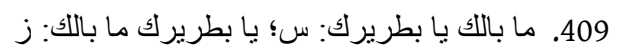

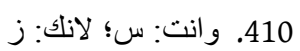

$$
\begin{aligned}
& \text { 411. الروم: س؛ الروم وتستهذهم الى قصدنا وتطمعهم فينا: ز }
\end{aligned}
$$

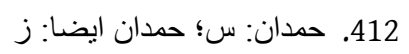

$$
\begin{aligned}
& \text { 413. فتحرك ذلك: س؛ فن فنض ابن ابن مانك: ز ز }
\end{aligned}
$$

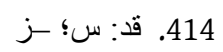

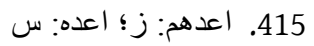

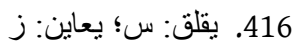

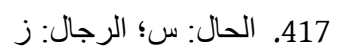

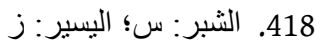

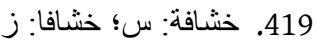

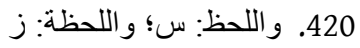

$$
\begin{aligned}
& \text { 421. استذعاهم: ز؛ استذعاه: س سل زل }
\end{aligned}
$$

Al- 'Ușūr al-Wusțā 29 (2021) 


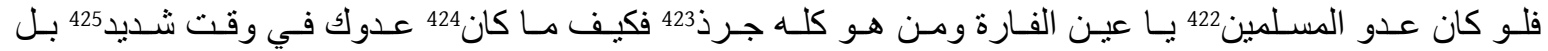

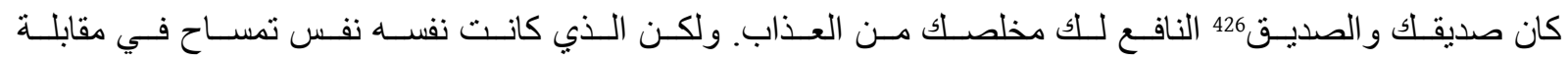
الخير بشر. 427 لما امر في بابه428 بما امر مد ذبح 429 المسيح عنقه وهو جالس بغير انزعاج منتظر ا اخذ راسه.

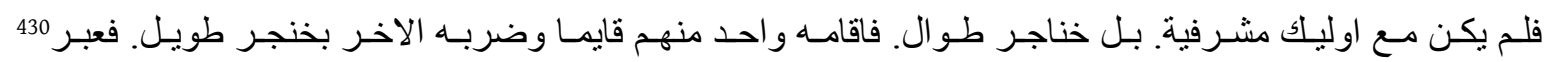

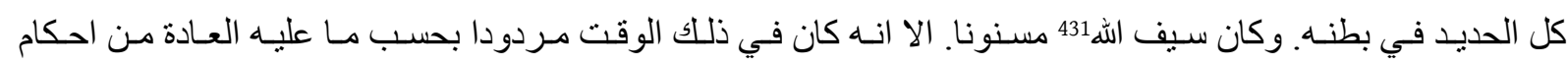

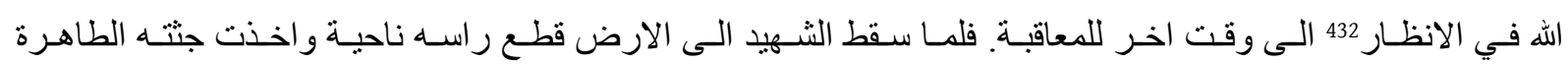

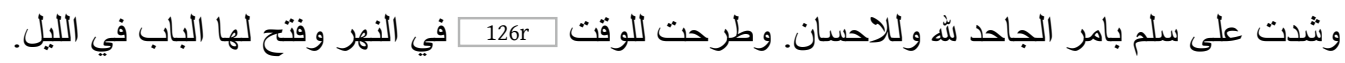

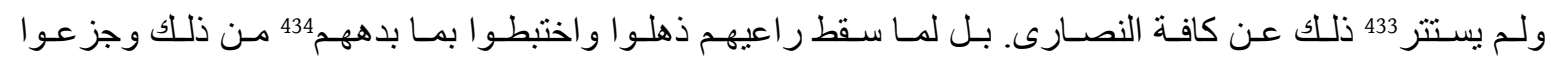

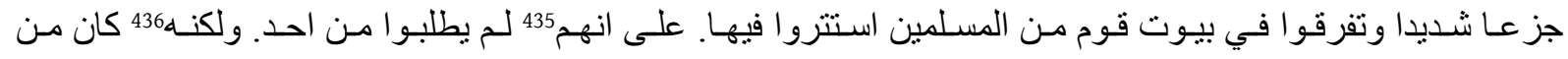

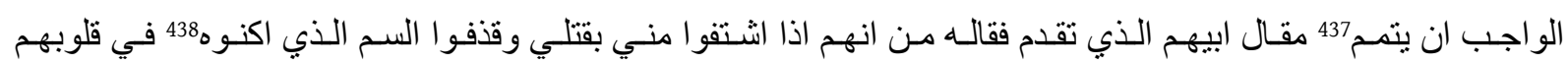
علي فلن بطلبوا سواي.

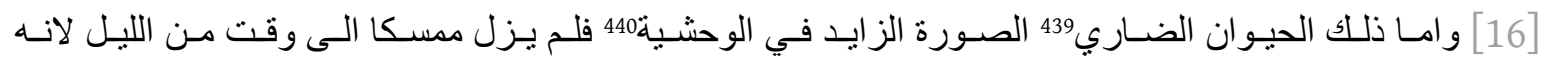

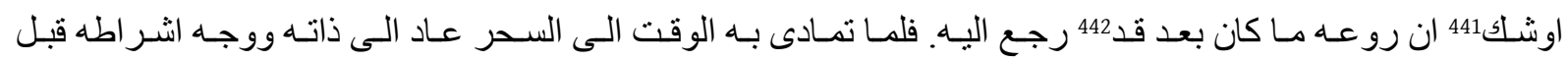

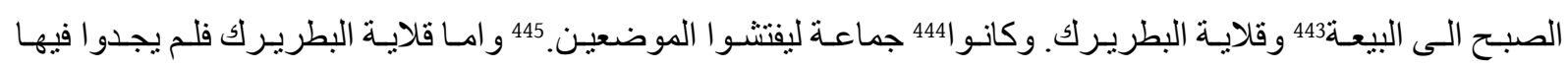

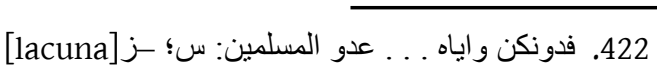

$$
\begin{aligned}
& \text { 423. جرذ: س؛ جود: زل ز }
\end{aligned}
$$

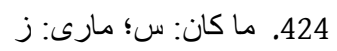

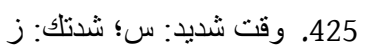

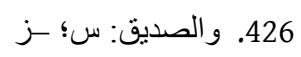

$$
\begin{aligned}
& \text { 427. بشر: س؛ بالثر: ز ز }
\end{aligned}
$$

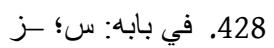

$$
\begin{aligned}
& \text { 429. ذبح: س؛ نبان: شروف: ز ز }
\end{aligned}
$$

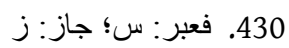

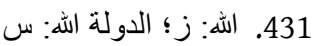

$$
\begin{aligned}
& \text { 432. الانظار: س؛ الانتظار: ز ز }
\end{aligned}
$$

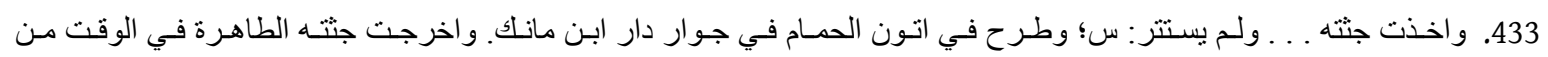

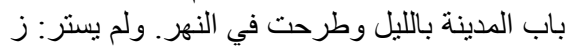

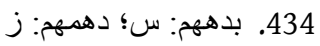

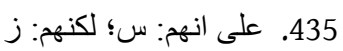

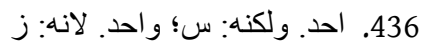

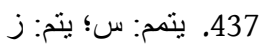

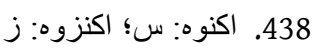

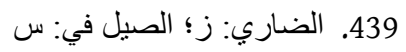

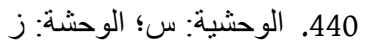

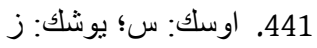

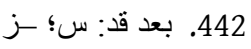

443. البيعة: س؛ البيعة التي هي كنيسة القسيان: ز

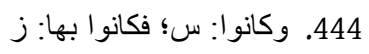

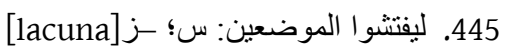




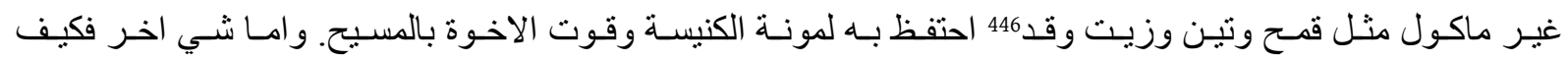

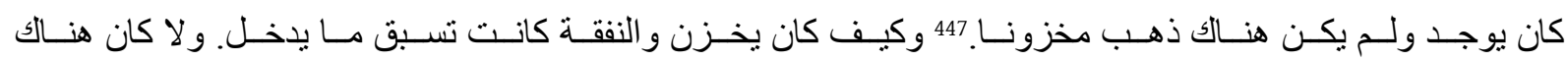

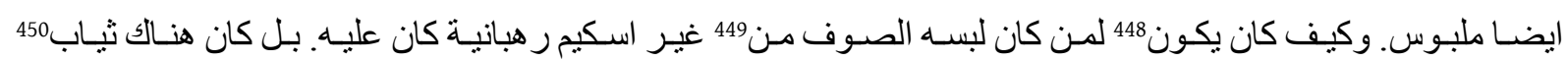

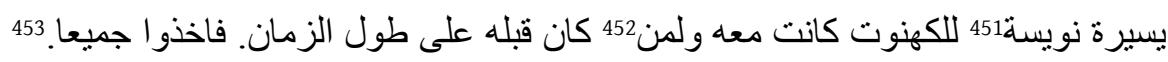

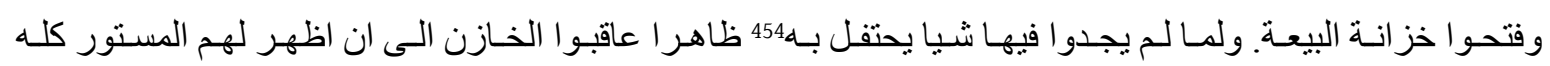

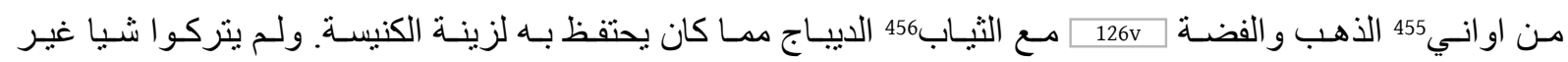

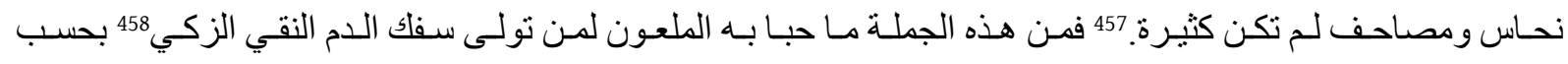

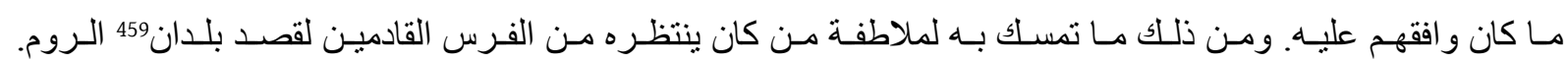

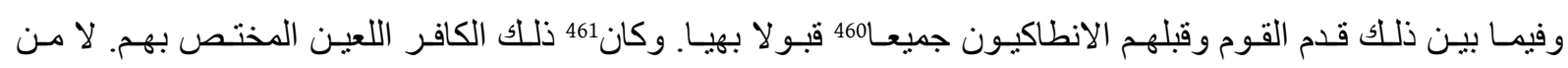

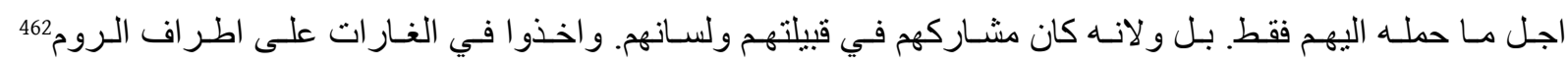

واجتياحها.

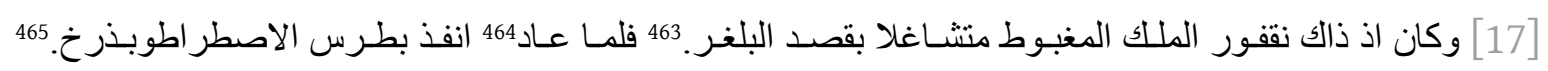

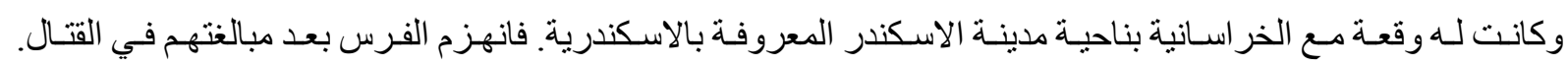

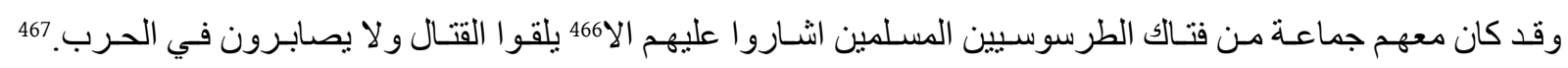

$$
\begin{aligned}
& \text { 446. قمح وتين وزيت وقد: س؛ تين وزبيب قد: ز }
\end{aligned}
$$

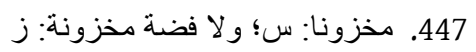

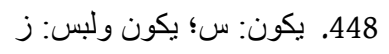

$$
\begin{aligned}
& \text { 449. من: س؛ - نز } \\
& \text { 450. ثياب: س؛ انشياء: ز ز }
\end{aligned}
$$

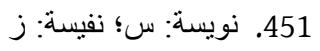

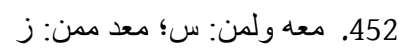

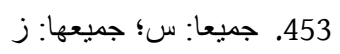

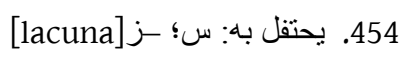

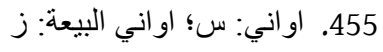

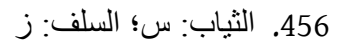

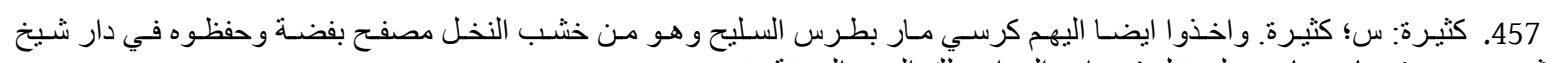

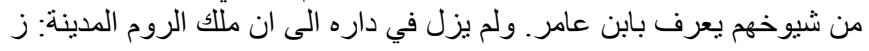

$$
\begin{aligned}
& \text { 458. الزكي: س؛ الذكي جنسه: ز }
\end{aligned}
$$

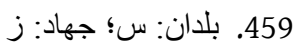

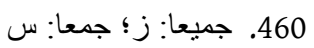

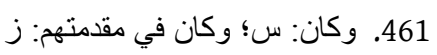

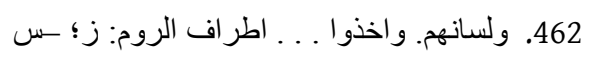

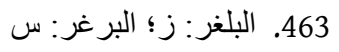

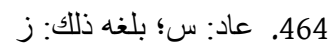

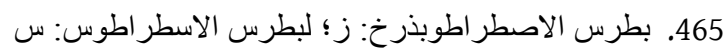

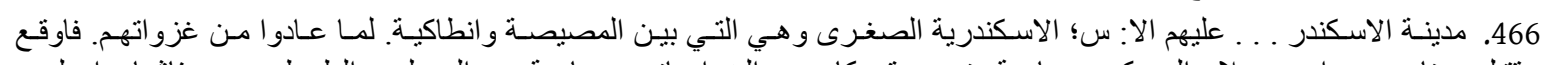

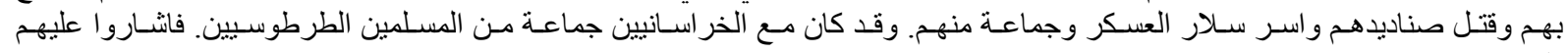


اذ قالـوا. ان هذا الجيش الذني يقاتلكم عسكر ملكي وليسـت468 لكم بهم طاقـة. فلم يقبـل منهم الفرس. بـل صابروا الصى

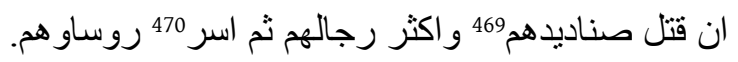

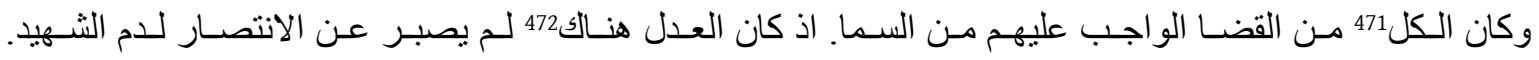

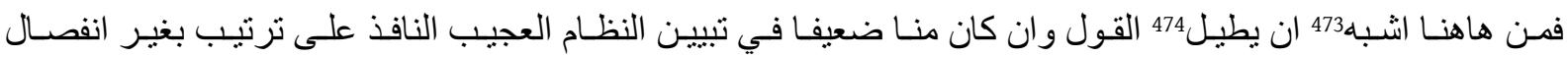

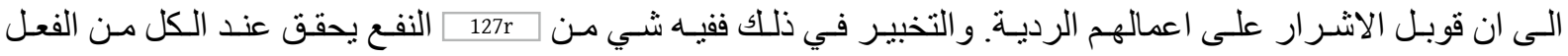

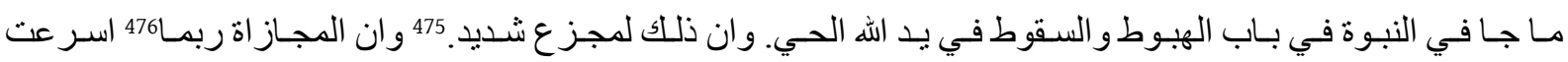

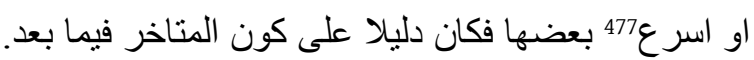

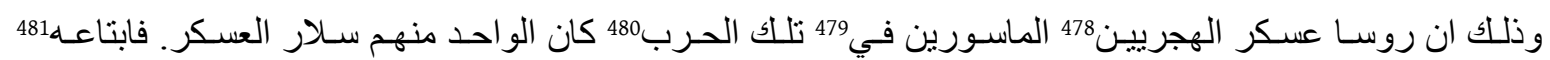

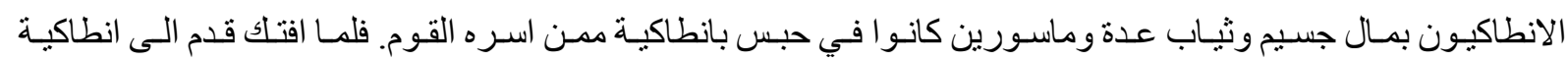

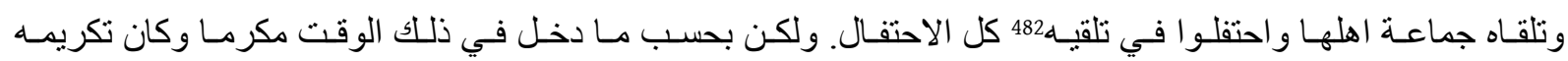

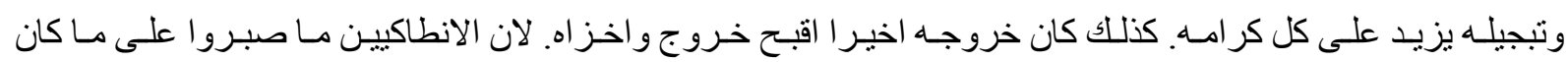

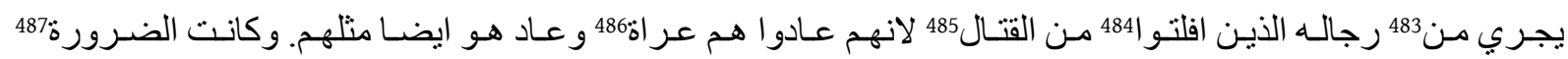

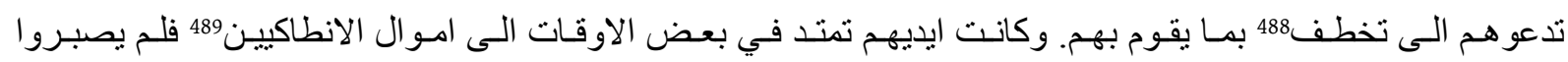

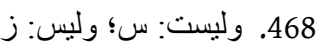

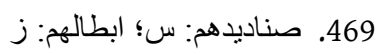

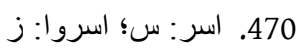

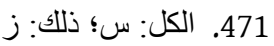

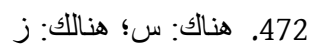

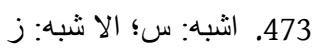

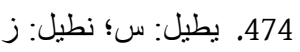

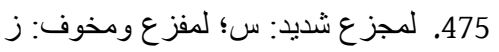

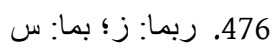

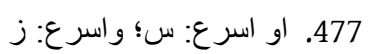

$$
\begin{aligned}
& \text { 478. روسا عسكر الهجريين: س؛ رؤس رؤساء الخراسانيين: ز }
\end{aligned}
$$

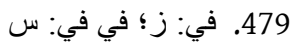

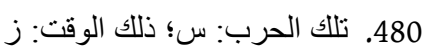

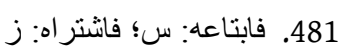

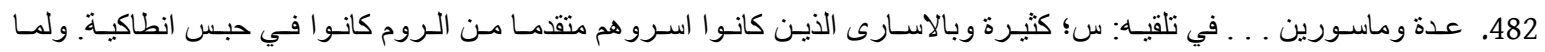

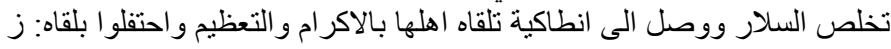

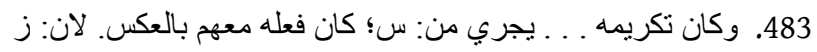

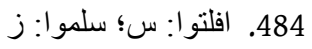

485. 285 القتال: س؛ القتل: ز ز

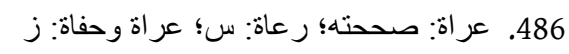

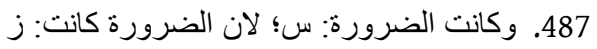

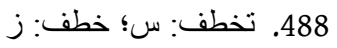

489. وكانت ايديهم . . . امو ال الانطاكيين: س؛ فتسلطو ا على الانطاكيين وصاروا يتخطفو ا امو الهم ورحالاتهم: ز 
لهم 490 على ذللك. بل دافعو هم وانتشبت491 الحرب فيما بينهم واخرجهم اهل 492 المدينة عراة.

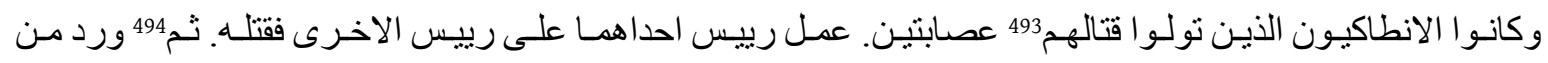

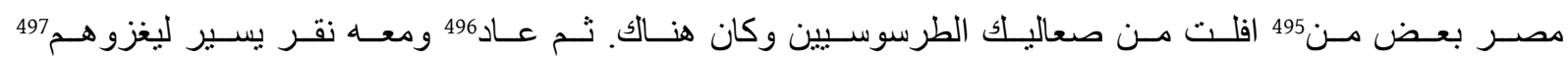

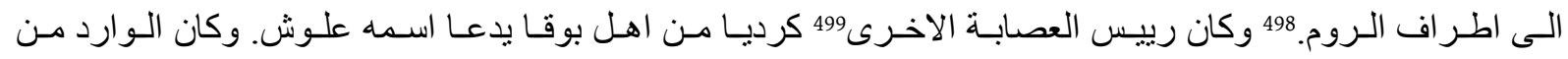

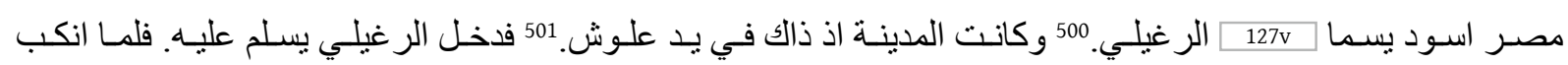

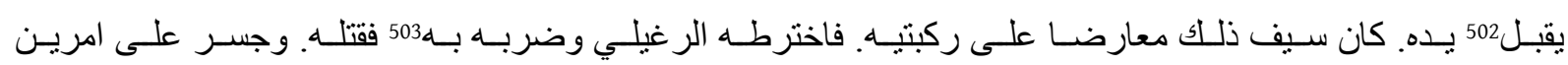

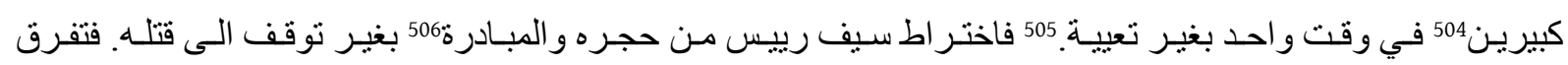

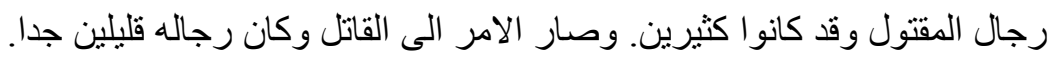

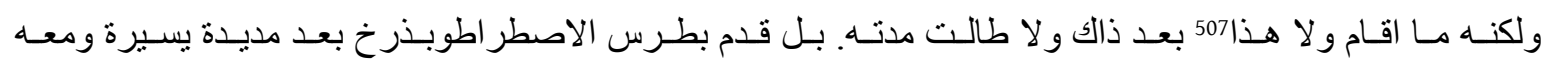

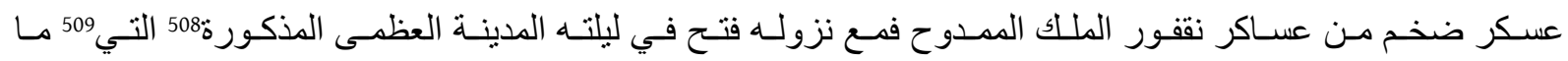

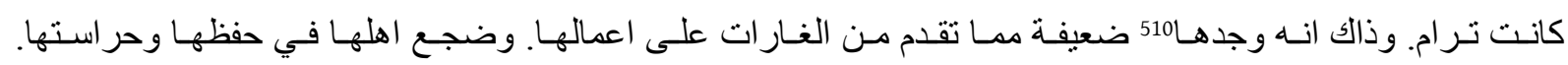

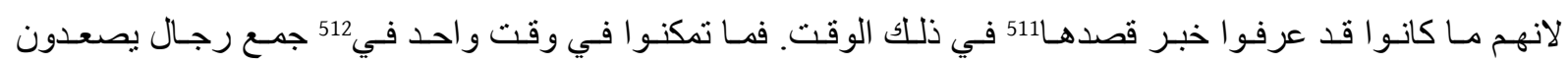

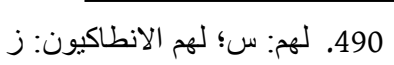

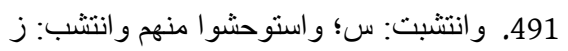

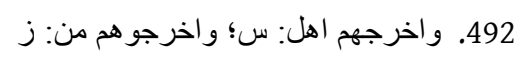

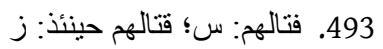

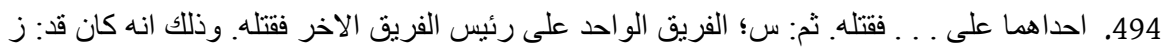

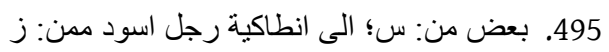

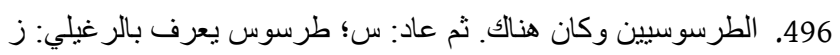

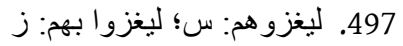

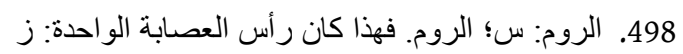

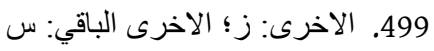

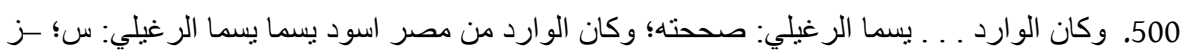

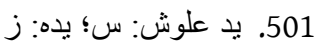

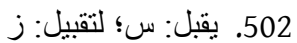

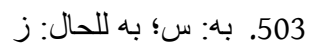

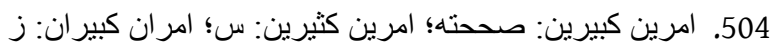

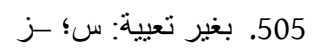

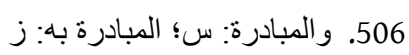

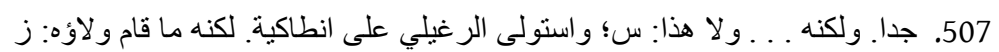

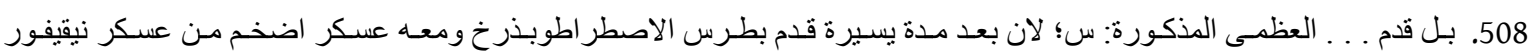

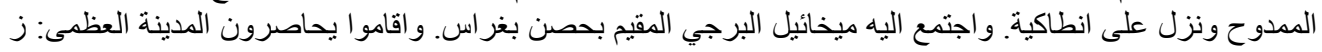

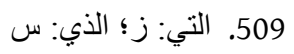

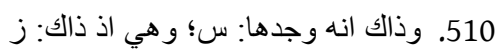

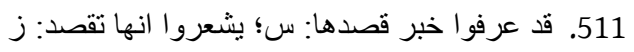

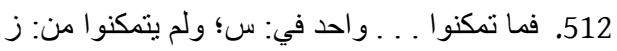

Al- 'Ușūr al-Wusțā 29 (2021) 


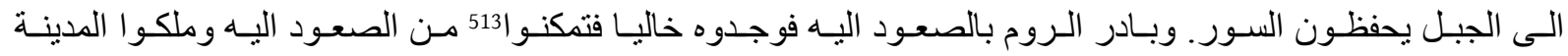

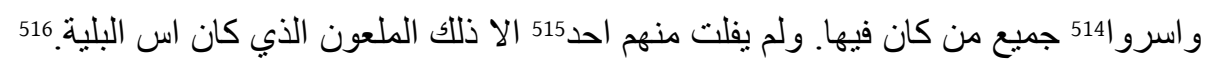

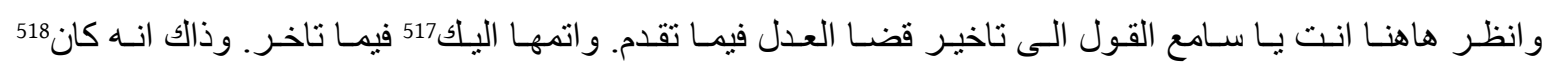

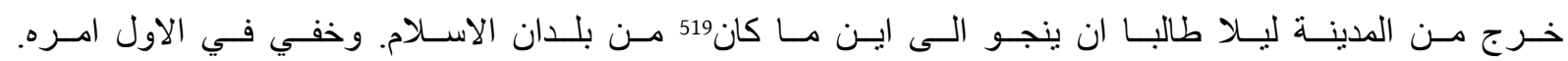

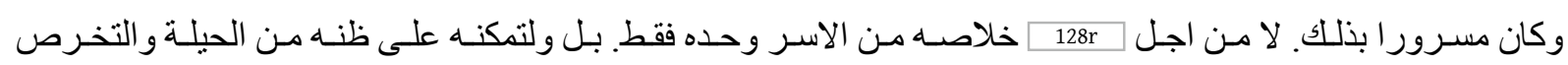

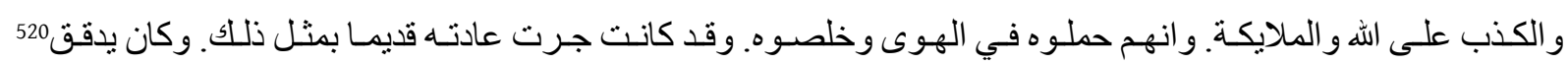

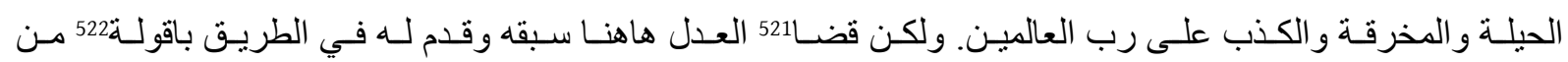

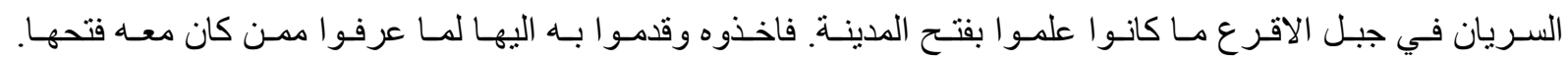

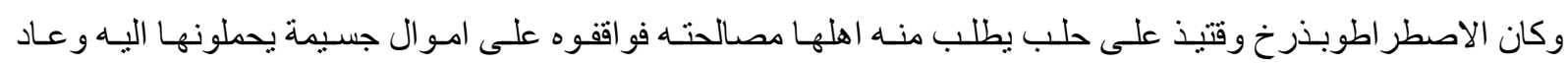

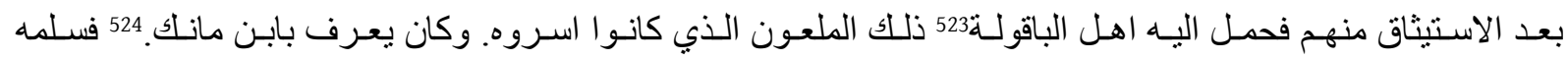

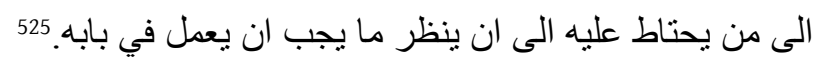

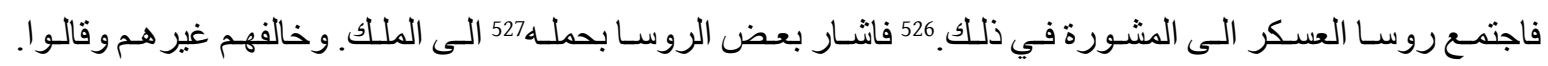

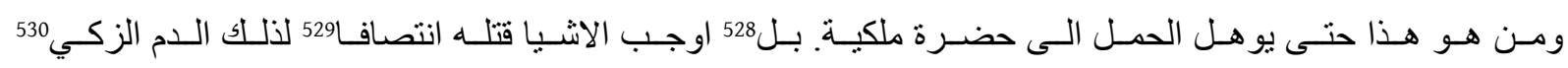

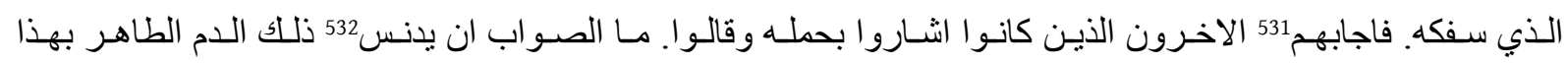

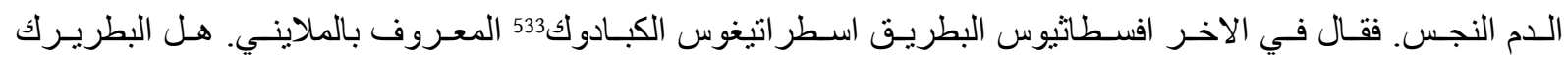

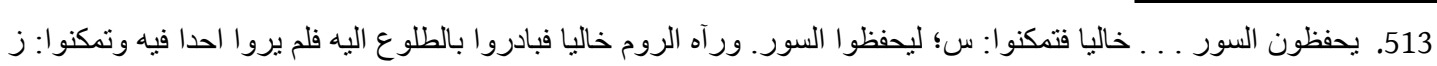

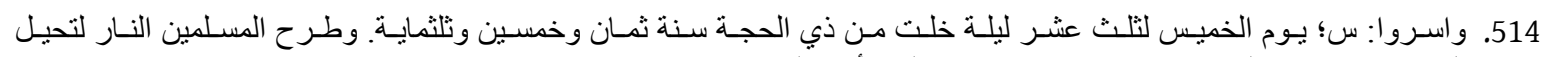

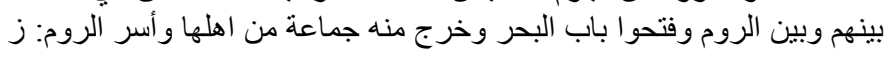

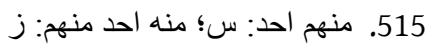

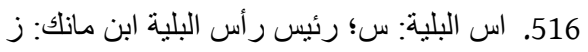

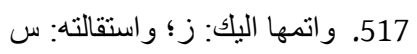

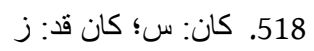

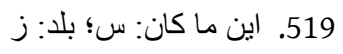

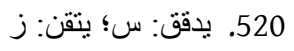
521.

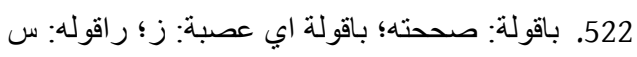

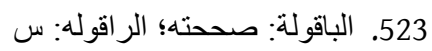

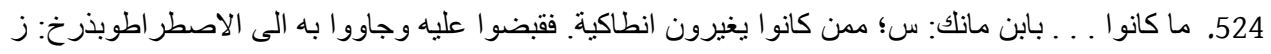

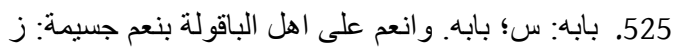

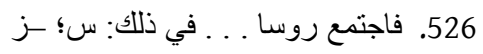

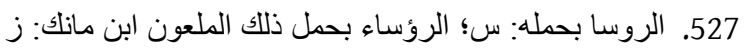

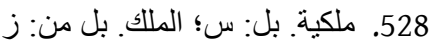

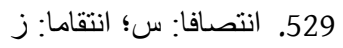
530.

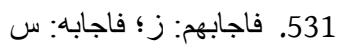

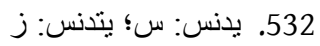

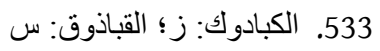




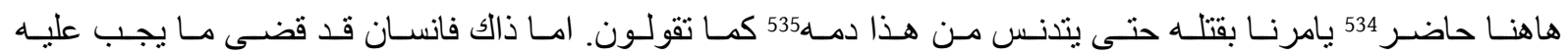

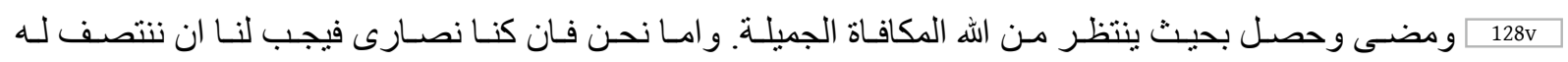
من الظلم الجاري عليه.

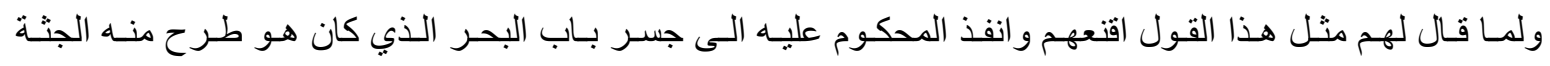

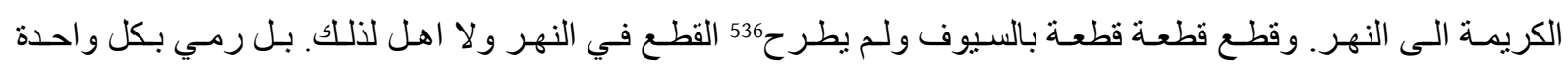

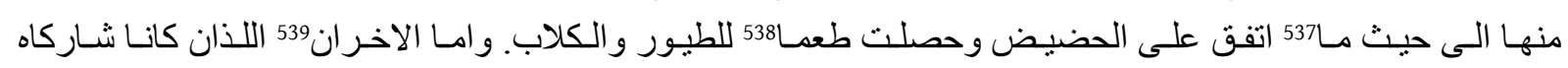

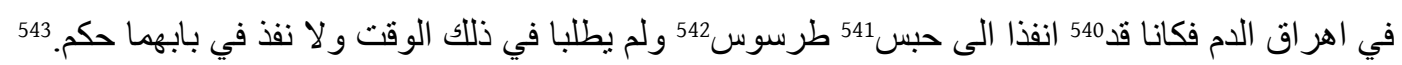

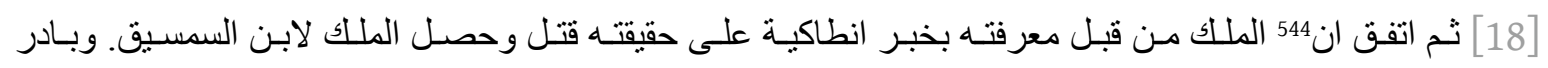

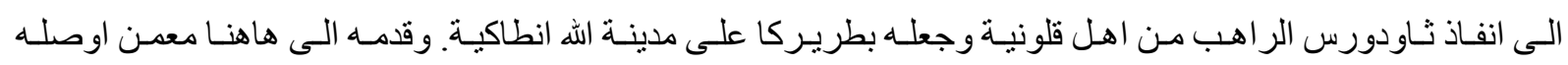

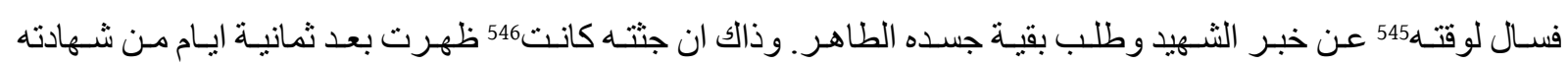

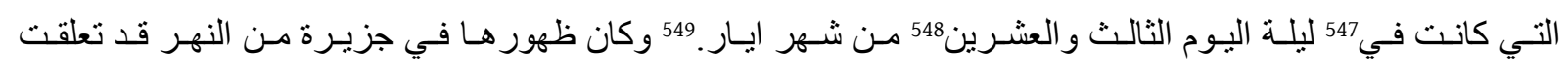

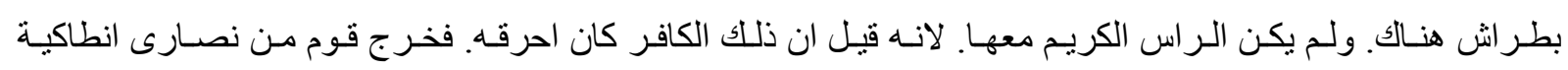

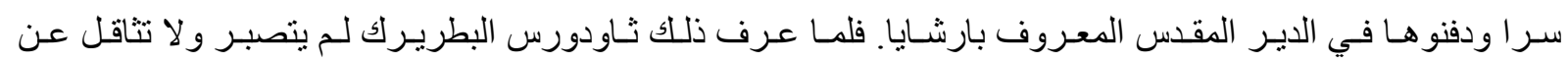

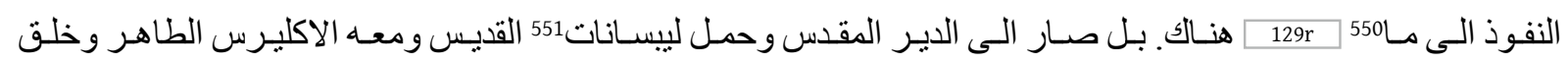

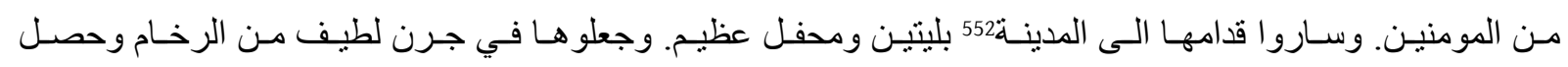
على مايدة503 رخام في مغارب الكنيسة الكبرى.

534.

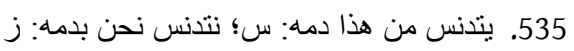

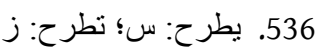

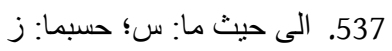

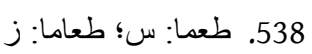

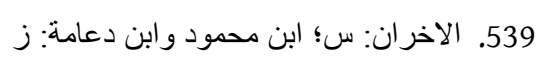

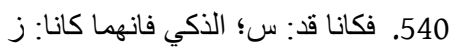

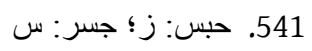

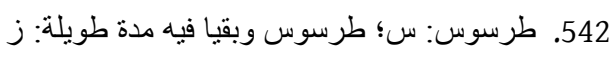

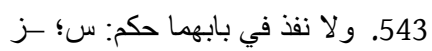

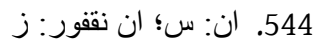

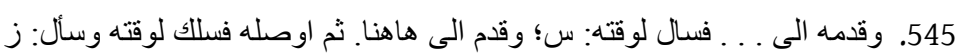

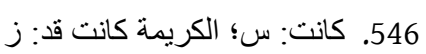

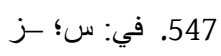

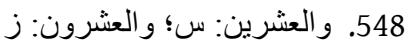

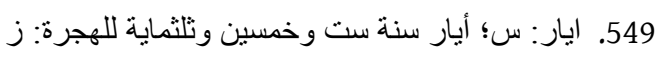

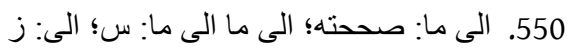

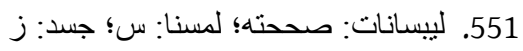

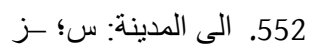

553. وحصل على مايدة: س؛ ووضعبة سل سلى على مائدة من: ز

Al-'Ușūr al-Wusțā 29 (2021) 
الا ان انبـا54 نقو لا البطريـرك مـن بعـد برهـة مـن السنين نقلـه مـن هنـاك باكر ام ايضـا. وحصلــ555 في جـوف بيت

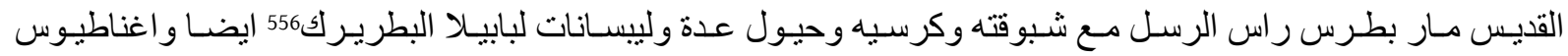

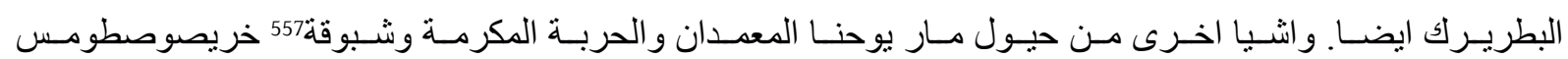
ومنطقة مار سمعان الحلبي العمودي. وغير ذللك مما هو كله في خزانة القديس مار بطرس الى غايتنا هذه.

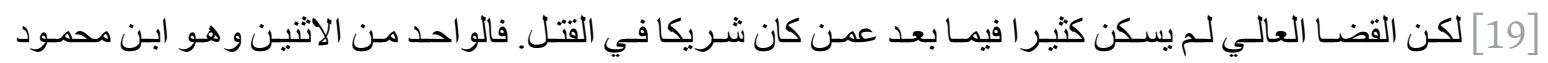

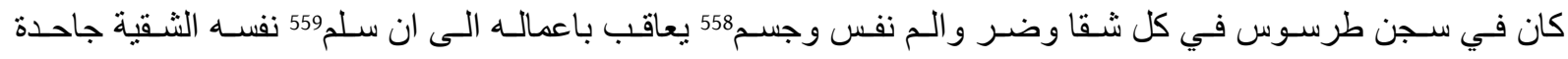

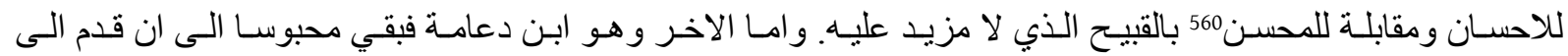

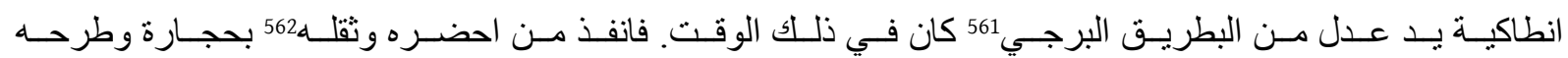

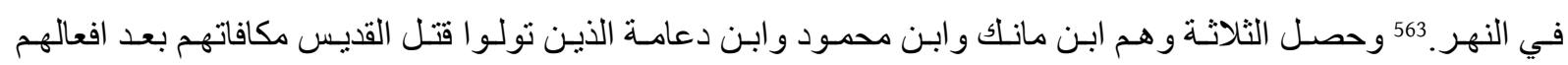
وفي 564 الاخرة ينتظرون العقوبة الدايمة.

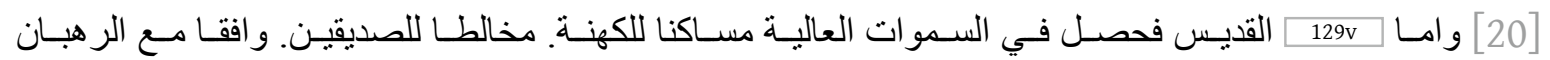

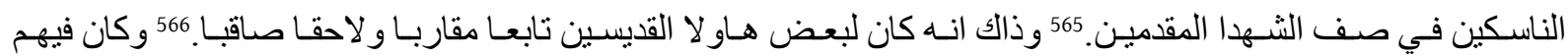

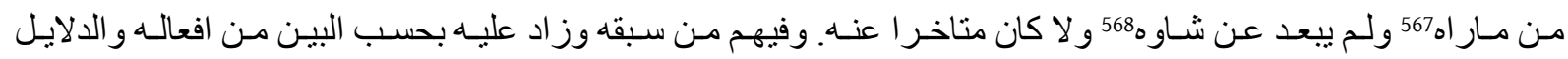
المعروفة من اعماله.

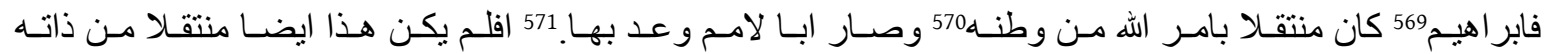

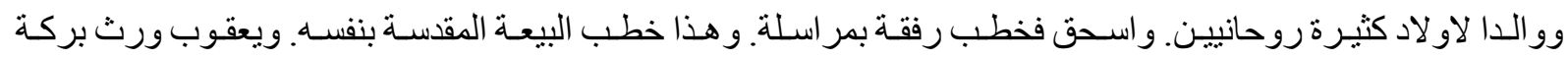

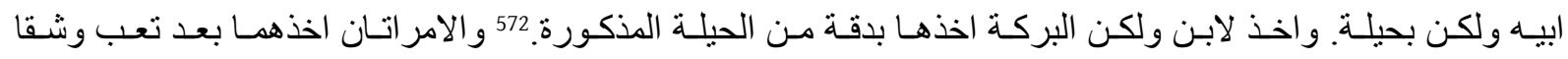

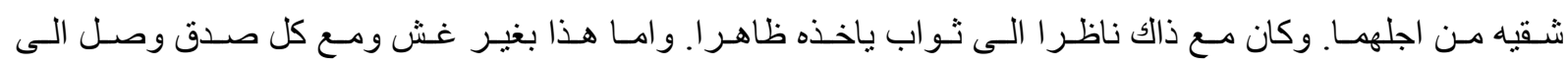

$$
\begin{aligned}
& \text { 554. 5 انبا: س؛ القديس: ز ز }
\end{aligned}
$$

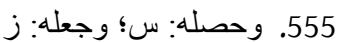

$$
\begin{aligned}
& \text { 556. ولييسانات لبابيلا البطريرك: سي؛ ولبه: ولباسات للآباء البطاركة: ز }
\end{aligned}
$$

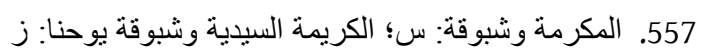

$$
\begin{aligned}
& \text { 558. نفس وجسم: س؛ يقين وجسيم: ز }
\end{aligned}
$$

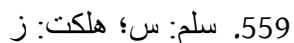

$$
\begin{aligned}
& \text { 560. جاحدة للاحسان ومقابلة للمحسن: س؛ المقابلة للحسن: ز زلئ }
\end{aligned}
$$

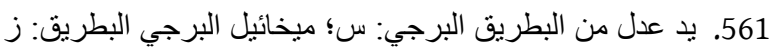

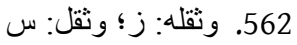

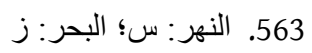

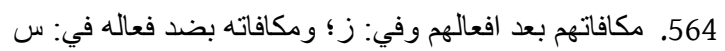

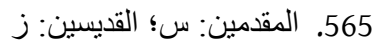

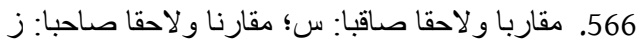

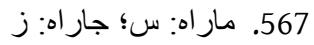

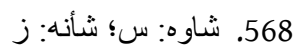

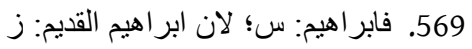

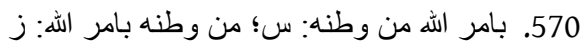

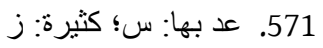

$$
\begin{aligned}
& \text { 572. و واخذ لابن . . . الحيلة المذكورة: سك؛ -ـز }
\end{aligned}
$$




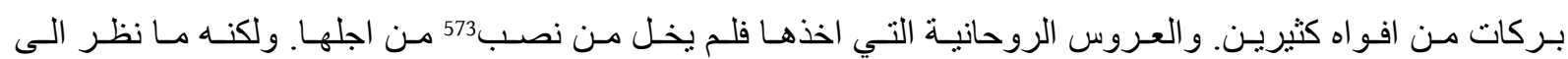

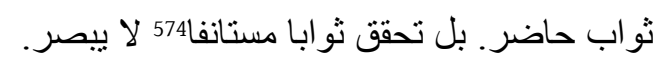

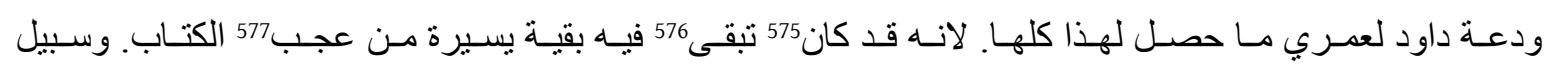

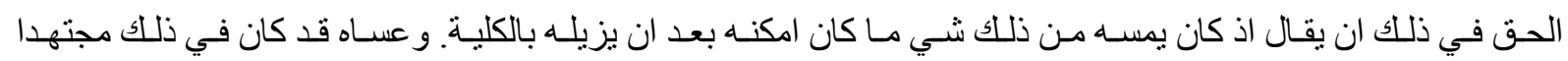

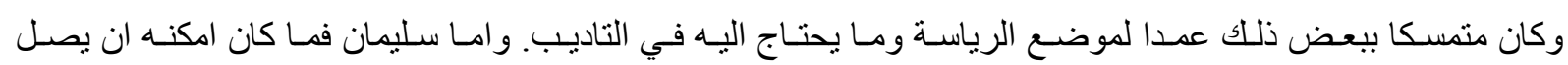

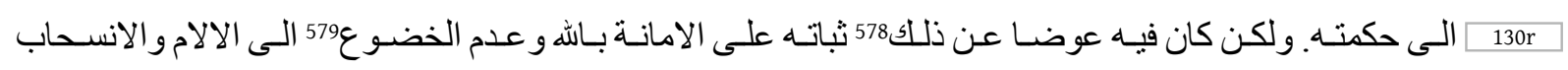
الى الخنى. و هذا580 فهو اشرف من حكمة الدنيا واثر عند الله.

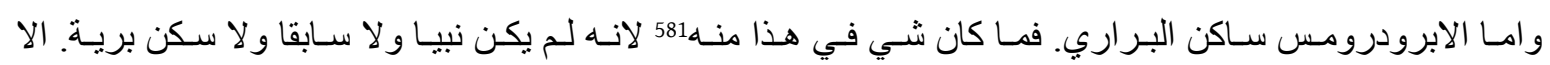

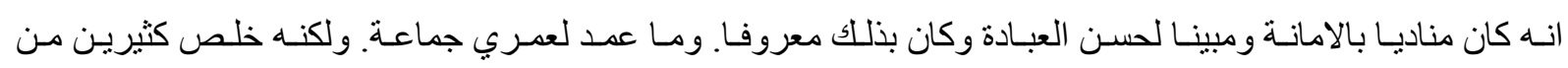

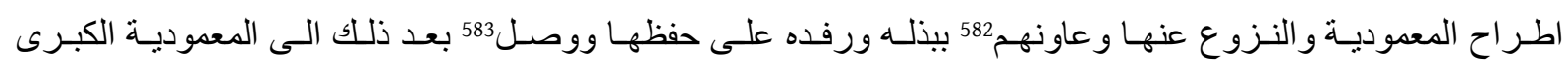

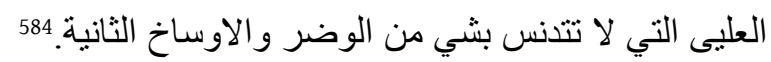

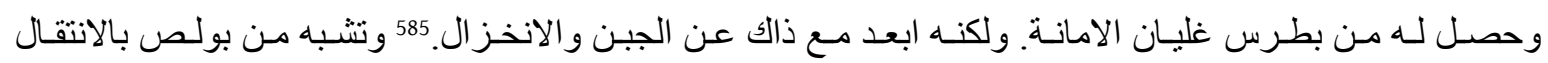

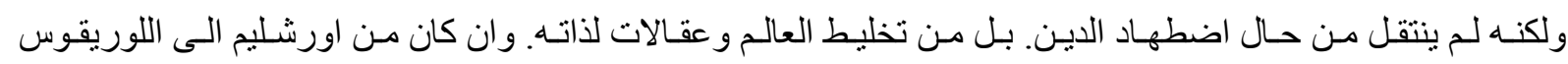

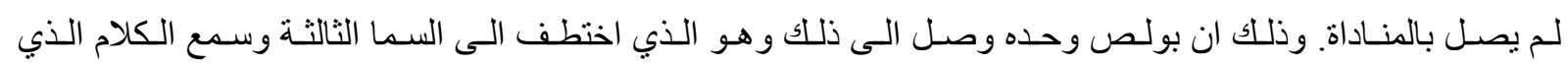

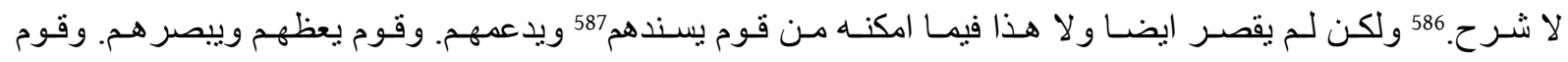

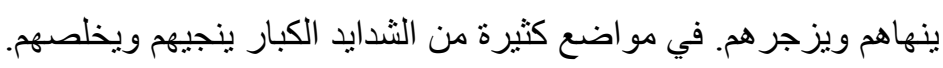

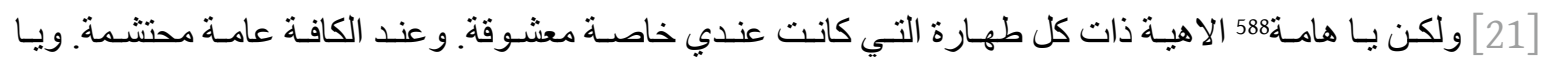

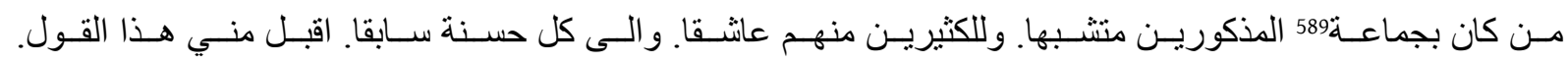

$$
\begin{aligned}
& \text { 573. نصب: س؛ تعب: ز }
\end{aligned}
$$

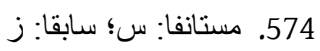

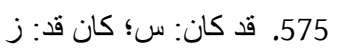

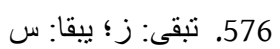

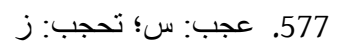

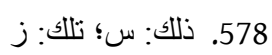

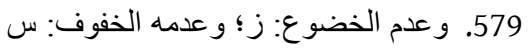

$$
\begin{aligned}
& \text { 580. الخنى. و هذا: س؛ الحياة. و هذا لعمري: ز ز }
\end{aligned}
$$

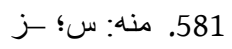

$$
\begin{aligned}
& \text { 582. و النزوع عنها و عاونهم: س؛ و الترو غ عنها و عادتهم: ز }
\end{aligned}
$$

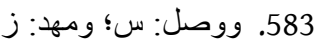

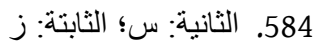

$$
\begin{aligned}
& \text { 585. ذاك عن الجبن و الانخز ال: س؛ ذلك الك الجبن و والانحر الك: ز }
\end{aligned}
$$

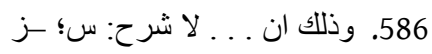

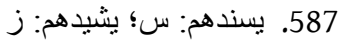

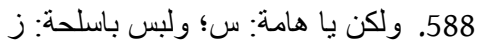

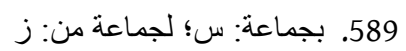




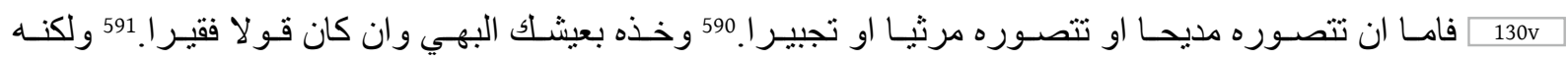

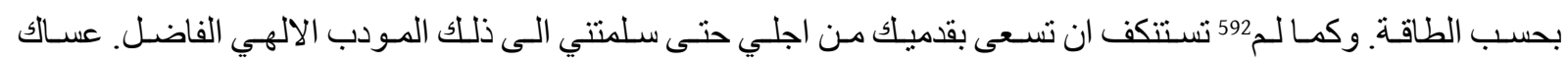

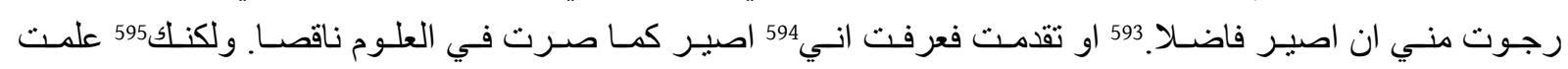

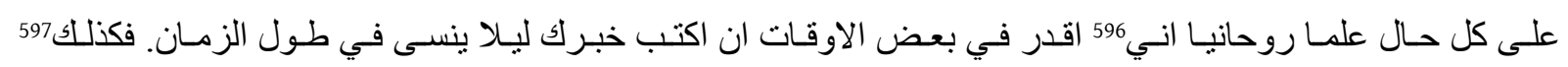

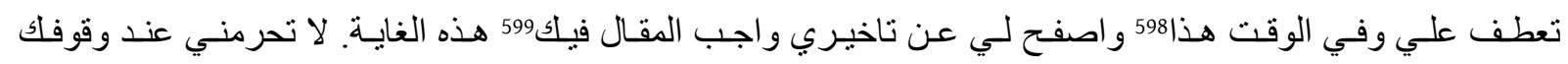

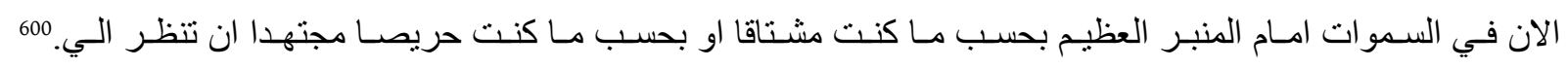

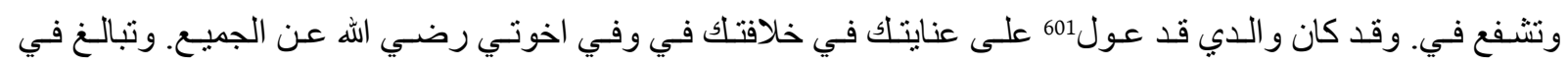

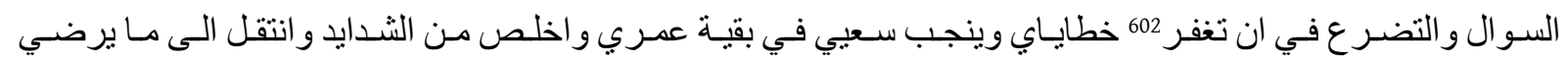
الله ويزلف لديه.

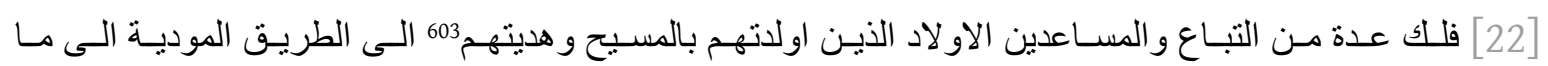

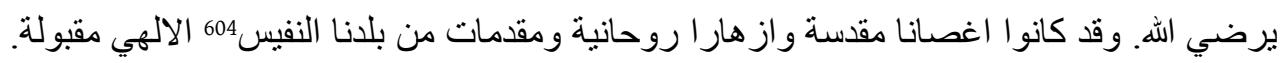

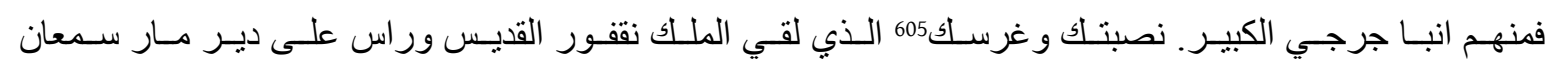
العمودي الحلبي الجبلي.

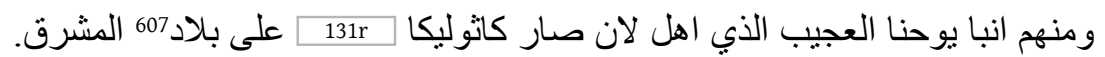

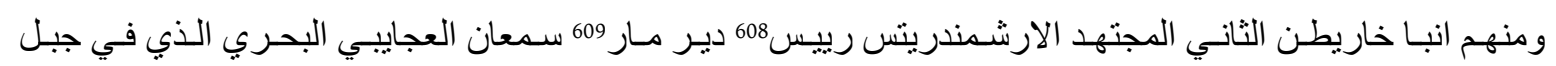
اللكام العجيب.

$$
\begin{aligned}
& \text { 590. مديحا او . . . او تجبيرا: س؛ موفقا او تتصوره مرتبا او تخبيرا: ز }
\end{aligned}
$$

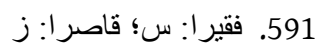

$$
\begin{aligned}
& \text { 592 5. لم: ز؛ لا: سل سل } \\
& \text { 593. اصير فاضلا: س؛ اصف لإن واصبر قليلا: ز }
\end{aligned}
$$

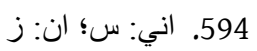

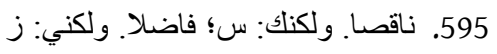

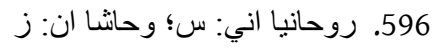

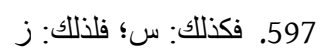

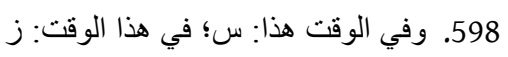

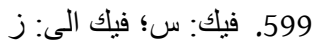

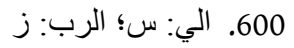

$$
\begin{aligned}
& \text { 601. كان و الدي قد عول: س؛ كانوا الذين عولو ا: ز }
\end{aligned}
$$

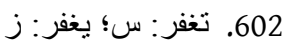

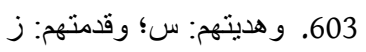

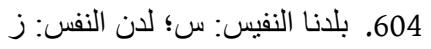

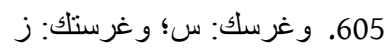

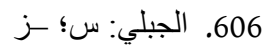

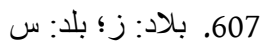

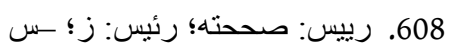

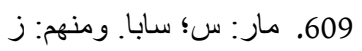


ومنهم انبا يعيش الحبيس610 الذي جاهد في 611 انواع من عمد الحبس وصنع القوات العجيبة.

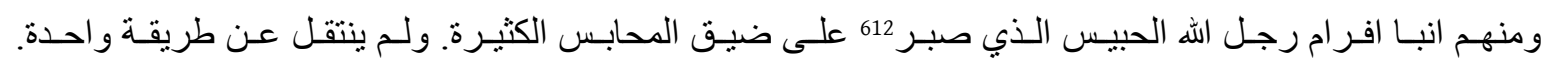

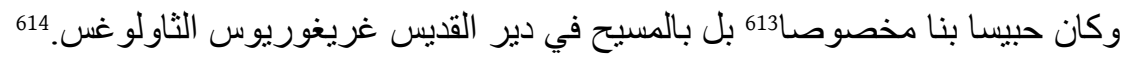

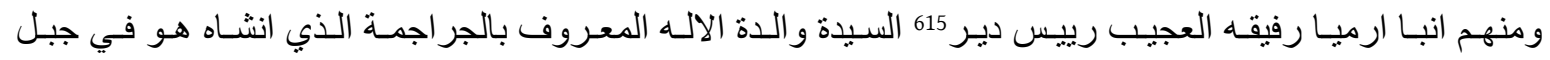
اللكام المقدس. ومنهم الاب المغبوط افتيكيوس ابن فرخوس.616

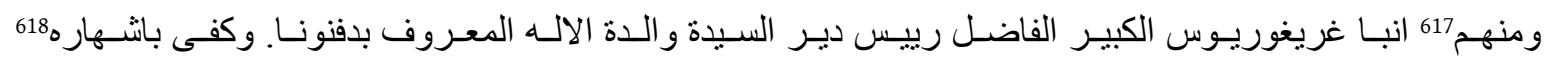
في الفضايل.

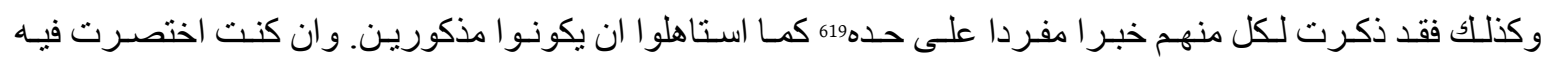

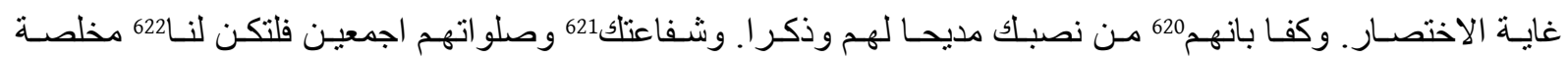

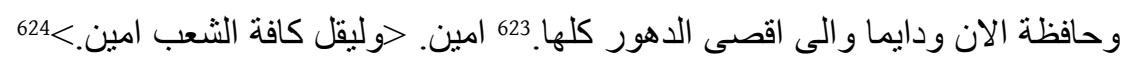

$$
\begin{aligned}
& \text { 610. الحبيس: س؛ الحلبي: ز }
\end{aligned}
$$

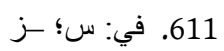

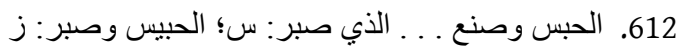

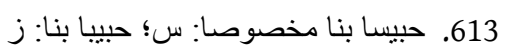

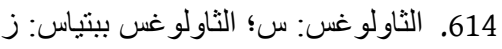

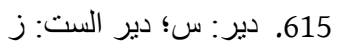

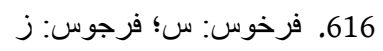

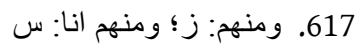

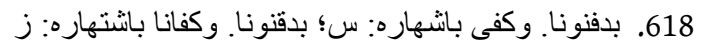

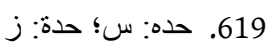

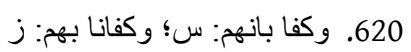

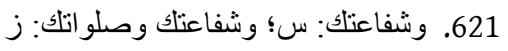

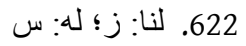

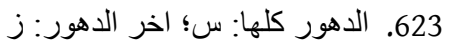

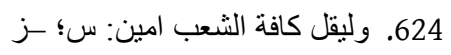




\section{Translation}

111v In the name of the Father and the Son and the Holy Spirit, one God.

The twenty-third day of May.

The story of the life of the patriarch of Antioch,

the martyr Christopher, and his martyrdom there.

Ibrāhīm b. Yūhannā, the imperial ${ }^{625}$ prōtospatharios there, composed it in Greek, then also translated it into Arabic. May God benefit us by it, amen.

Nature and actions in accordance with the divine image ${ }^{626}$ are among the deeds of God (blessed be every mention of him). As for sin and all things that diverge from the outlines of that image, they are among the deeds of the Evil One.

And as for our own deeds, we say first that they are among the deeds of God, because everything recognizes that he (exalted be his name) created all things that exist out of nothing and ennobled humans by his own hand when he created them in his image. But we mention secondly that they are among the deeds of Iblīs, because he envied our father Adam for the nobility that he had attained, so he deceived and misled him with the hope of divinization, making him sink and fall away from the natural virtues and outstanding traits of the image upon which he was formed. Thus $112 \mathrm{r}$ it has become a virtue to establish our nature upon its original foundation and basis and to put an end to our separation from the core of the image, and it has become a shortcoming and a vice to remove ourselves from the duties of our nature, to turn away from its original basis in any direction, to abandon the image's journey toward that upon which it was formed, and to turn our faces toward its opposite.

Thus the prophet's saying "I will judge you according to the state in which I find you at the end" 627 became necessary. By my life, this is one of the most necessary and important things! For God (blessed be his name), who is the first good, made us and created us to do good, so that good might be our goal and intention. After we have deviated from it, we return to it, so that we might begin to ascend toward it after turning away, because the only thing that prevents us from doing so is not God (powerful and great), but our own will. How, then, could this saying not be necessary? From this it follows that he will judge us according to the state in which we are found at the end of our days. So we have come to this point: Whomever we see in a given form at the end, whether they have been good and

625. "Imperial" (malakī) could also be translated "Melkite" in a reference to Ibrāhīm's Christological affiliation. However, compare MS British Library Or. 8607, fol. 28b, where an Abraham (possibly this author) is described as "the emperor's scribe," kātbā d-malkā. See Brock, "Syriac Manuscripts," 62, 66-67. For more on this translation choice, see Mugler, "Ibrāhīm ibn Yūhannā," 192-93; Samuel Noble, "A Byzantine Bureaucrat and Arabic Philosopher: Ibrāhīm ibn Yūhannā al-Anțākī and His Translation of On the Divine Names 4.18-35," in Caught in Translation: Studies on Versions of Late Antique Christian Literature, ed. Madalina Toca and Dan Batovici, 276-312 (Leiden: Brill, 2020), 268-69.

626. The preface uses the roots $s-W-r$ and $s-y-r$ ("image," "become," etc.) as a running motif.

627. Ezekiel 7:3? 
righteous all of their days or have returned to goodness and righteousness after straying far away-they have returned, and we must praise and commend them, because we know that nothing prevented them and they have turned toward this form of power without anything to hinder them, unto the ultimate blessing and the furthest remembrance.

When we find someone who first made a mistake and then apologized for it in the end, it is no embarrassment to praise and exalt them on account of what they became afterward. So the "chosen vessel," ${ }^{28}$ despite his previous $112 \mathrm{v}$ acts of persecution, must be highly praised, since his struggle ${ }^{629}$ ended on the side of truth. The same applies to Matthew, because he became an evangelist after collecting taxes. ${ }^{630}$ So if anyone is like these two, who first went one way but afterward returned to virtue, they must be praised for what they began to do afterward. And if this is true of those who were in error before, what can we say about one who was not in error before, but was rather in the middle ranks of people, among those who were polluted voluntarily or involuntarily, between the highest and the lowest? The person who fits this description must certainly be considered virtuous and counted among those who should be greatly praised.

\section{[1. The Early Years]}

One person we know who fits this description is Christopher, ${ }^{631}$ the great patriarch and the noble martyr, because of the payments he received before, when he was a secretary, ${ }^{632}$ and because he endured his later struggle and devotion, though he did not have an earlier custom or precedent of religious exercise. He had a powerful passion and zeal for even the smallest good works and later had an even greater passion for struggling on behalf of the truth.

His homeland was the City of Peace, Baghdad, which the early children of Hagar substituted for the city of Ctesiphon as their capital city, the land of their sciences and their pure language. As for his parents, I do not know what they were like, but they must have been prominent and important to become the parents of someone like him. The period of his education in his own city was brief, consisting only of what he needed to become well educated in the elementary sciences, then to master rhetoric as well as he could, and beyond that to master the skill of handwriting. For when he wrote, no $113 \mathrm{r}$ one could decide which was more perfect in his calligraphy: its beauty or its speed. For these two qualities had never been equally present in a single person. In him, not only were they equal, but everyone who saw him thought that they were competing with each other and striving for precedence.

628. Saul/Paul; Acts 9:15.

629. Jihād.

630. Cf. Matthew 9:9.

631. The text of $Z$ begins here and reads "Christopher, the blessed, fortunate one."

632. Z omits: "because of ... a secretary." 
For this reason, and due to his aforementioned virtue-or rather, due to the concern of divine providence for the see of the Head of the Apostles ${ }^{633}$ that is here-it was not possible for him to dwell in his homeland for long. Once he had become as well educated as he could be, as we have mentioned, he imitated the chosen father ${ }^{634}$ who had migrated from those regions; he, too, migrated, because he, too, was going to become the great father of a multitude. He migrated from the land of the Chaldeans to the land of the Syrians. And by "the land of the Syrians," I do not mean "the land of the Canaanites," which is now the land of Palestine, but I mean the town near us, not far away, the town of Aleppo. The management of all of this was with God, who lays the foundations of things in advance, so that at last they come to a praiseworthy end beneficial to all. He (great and powerful) continuously makes small things the prologue to great things that do not even occur to the imagination beforehand.

In this case, Ibn Hamdān, whose surname was Sayf al-Dawla and whose renown was great, was important and had a powerful influence in this region. He showed concern for every virtuous person who had nobility and high ambition, so he was like a magnetic stone whose goodness attracted ${ }^{635}$ people from $113 \mathrm{v}$ all other countries. Therefore, the one who had imitated the ancient father in his migration headed in his direction. His name was originally 'I $\bar{s} \bar{a},{ }^{636}$ and he was going to become what he was going to be named-or rather, what he was going to be named came first, so he took that name. ${ }^{637}$ And just as he had put on Christ in baptism, ${ }^{638}$ so he would also put him on in his blood.

'Alì b. Hamdān handed him over to one of the great emirs of the wilderness, named Khalīfa b. Jundī, whose emirate was in the area of Shayzar. So 'Īsā was with him, and all of his affairs were handed over to him.

\section{[2. The Christians of Iraq and Central Asia]}

He was not known among the Antiochians at first; no one had heard of him or knew anything about him, but afterward he became known because of the coming story. Ctesiphon, which we have mentioned, was large and greatly renowned, because it was the limit, frontier, and boundary of the kingdom of the Persians. And there was a community

633. Ra's al-salīhiyyīn, that is, Peter, whose first see was in Antioch.

634. Abram/Abraham, whose two names mean "great (or high) father" and "father of a multitude," respectively; cf. Genesis 11:31-12:9, 17:5.

635. Lacuna in Z omits: "like a ... goodness attracted."

636. The typically Muslim name for Jesus (the typically Christian name is Yasūc). Z adds: "because he was going to become a patriarch like Abraham, though he did not know it. But he was later named Christopher."

637. This sentence is confusing, and quite different in the two manuscripts. It seems, however, that the idea is that Christopher was going to live up to his name by becoming a bearer of Christ. The second part of the sentence suggests a correction to the first: he was already a bearer of Christ, and that was why he chose the name Christopher when he became patriarch. This assumes the audience's knowledge that the Greek name Christopher means "bearer of Christ," which would have been obvious in the original Greek.

638. Cf. Galatians 3:27. 
of Christians there whose pastor was not called a metropolitan, because the rank of metropolitan was not sufficient to shepherd such a large group throughout all the countries of Persia and to hand over their affairs to bishops alone. For they were also rather few, and the situation called for numerous bishops, so many that it was not possible for one metropolitan to name and ordain them all, so there was a need for numerous metropolitans. Moreover, those regions were distant, far from our city called by the name of God, ${ }^{639}$ and they were in the kingdom of the Persians, which often prevented travel to $114 \mathrm{r}$ Antioch for the appointment of a metropolitan for every place.

There was already another policy among the ancients for this situation, the likes of which had been used for different nations and distant places, such as the Georgians, ${ }^{640}$ the Abkhaz, and the Bulgars: a person was ordained whose authority was greater than the authority of a metropolitan, and he was called a catholicos, as they were called in those places that we mentioned. Our predecessors followed this custom, and the leader of our city ordained a catholicos for the city of Ctesiphon.

When the Hagarenes ${ }^{641}$ built the City of Peace, which is Baghdad, they wanted to move the Christians away from its vicinity, so they moved them to a distant city in the Persian lands, called Shash, ${ }^{642}$ and sent the catholicos into exile there with his exiled companions. That relocated tribe was called "the community of the Romans," and naturally their group was called by this name. ${ }^{643}$

So the residence of the catholicos was in Shash for some time, and no one challenged or disputed it. But when a group of Roman prisoners began to accumulate and some of them obtained their freedom, a dispute began between the two sides. The group of Christians gathered in Baghdad said, "The catholicos belongs to us, and we are more deserving of him, because his residence was in Ctesiphon, and Ctesiphon is near us." And the people in Shash said, "We are the people of that place, who were moved from there to here with the catholicos of all the East, and as a person is nobler than any piece of land, it is necessary for us to have the catholicos, who moved here with us. We have precedence, as we are rational creatures, and it is more proper $114 \mathrm{v}$ for us than for you. You ask that you should have precedence on account of nothing but stones and dust!"

While the quarrel between the two sides was ongoing, the current catholicos died, and there was need for another person after him to carry out the ministry of the priesthood there. Therefore, three emissaries came to Antioch from Romagird requesting the ordination

639. A reference to Antioch's Greek title of Theou Polis, or "City of God."

640. For "Georgians," Z reads: al-Khazarān. There is no known catholicate among the Khazars, and Marius Canard suggests that Ibrāhīm intended Jurzān, the Georgians. This seems to be the reading in S, though it could also be read as Khurzān. See Marius Canard, "Une vie du patriarche melkite d'Antioche, Christophore († 967)," Byzantion 23 (1953): 561-69, at 562.

641. For "Hagarenes," Z reads: "children of Hagar."

642. Now part of Tashkent, Uzbekistan.

643. Apparently a reference to Rōmagird (Persian)/Rhōmagyris (Greek), the name of this Christian colony in Central Asia. The etymology of the name is disputed and difficult to reconcile with Ibrāhīm's jamā'at al-Rūm; see Néophyte Edelby, "Note sur la catholicosat de Romagyris," Proche-Orient chrétien 2 (1952): 39-46, at 40; Canard, "Vie," 563. 
of a catholicos. One was a priest and the other two were deacons. I, the speaker and writer, saw them-while I was still a boy-remaining in our church and saying that they would not return until they were given an audience and allowed to take the catholicos whom they had come from the end of the earth to request. And the aforementioned 'İsā heard they had come, and he was not pleased with their plan, because he was a Baghdadi and one of their opponents on the topic of the catholicos-in fact, the leader of that side. Working to put an end to their plan, ${ }^{644}$ he did not delay or rest, but left his work behind and took up the pain of the journey to Antioch to drive them away. There was no one who appointed him to do this; he took up the cause of his own accord, simply out of zeal for his homeland.

At that time, the one entrusted with the administration of our town named for God was the late Agapios ${ }^{645}$ b. Qa'barūn, who held the patriarchate before him. So the dispute, discussion, and quarrel over the installation of the catholicos took place in his presence, and the aforementioned 'I $\bar{s}$ a fiercely contended for the people of his country, presenting their arguments and seeking victory for them-to bring the catholicos to their city, which is the City of Peace and the replacement for Ctesiphon. $115 \mathrm{r}$. As the patriarch judged, he seemed not to be swayed by what 'Īsā was requesting, but he recoiled from his skill and was ashamed to face him, so he left the matter hanging. ${ }^{646}$ 'İsā was satisfied and departed, but he had shown the Antiochians that he was a man of great endurance and that he had a fierce zeal for the affairs of the church.

\section{[3. His Election to the Patriarchate]}

It was only a little later that the patriarch died, and it became the concern of the Antiochians to choose a patriarch and pastor to succeed him. For here, the choice did not belong to metropolitans and bishops-who care about nothing except what will improve their own situation, and do not care what will improve the situation of the masses-as is the custom in other places. Here, the choice was available to everyone affected; both commoners and elites cared about it and had a choice in it. Anyone who precisely considers the concept of choice will find that the system used here is unspoiled by personal desires, and therefore also pleasing to God.

When the Antiochians set about doing this, a group of them debated at length whether to choose one person or another. But they did not find anyone more agreeable to them or more suitable for their see than 'İsā. So when their community agreed to choose him, they brought their request to Sayf al-Dawla, because he was in command of the region. He was pleased with their position, because he was partial and favorable toward 'İsā. But he had no way to approve their choice, because he was wary of the impudence of the desert Arab whom he was serving, $115 \mathrm{v}$ a man of great ruggedness, boldness, and audacity. He had no doubt that the man would fight to keep 'Īsā, his secretary.

644. S omits: "because he ... their plan."

645. For “Agapios," S reads: “Agathon.” This is Agapios I (bishop 341-48/953-59).

646. For "he left the matter hanging," $\mathrm{Z}$ reads: "he closed the matter." 
It was good fortune from God, who (blessed be his name) willed for such a man to become patriarch here, that that Qarmați Bedouin ${ }^{647}$ went with Sayf al-Dawla on one of his raids. ${ }^{648}$ His horse slipped on Șārikha ${ }^{649}$ Bridge and he drowned in the Halys ${ }^{650}$ River. Then Sayf al-Dawla gave permission for the selection of 'Īsā, and the chosen one was quickly elevated to the exalted and God-honored see of the Head of the Apostles. He received ordination from the metropolitans, as is required. The ordination was splendid and dignified, and he naturally took the name Christopher, for it was obvious from his actions that he bore all goodness within his breast-more precisely, we could say that he bore Christ within his heart.

\section{[4. His Asceticism and Piety]}

From this point on he ${ }^{651}$ led him away from the path of ease and luxury onto another path, rough and difficult. For although he had not become a monk, he exceeded all monks in his practices: after his ordination, he tasted nothing of meat. Because he had no previous custom of following the monastic lifestyle, he was not satisfied with the things that his monastic predecessors had done, whether in fasting or vigils or rising for prayer. Rather, he exceeded and surpassed them in everything. He fasted every day from nighttime to nighttime, from the beginning of the year to the end, for from the time when he became patriarch, there was not a single day when he ate a bite of food before evening. Nor was he concerned about different types $116 \mathrm{r}$ of food; he made no distinctions among the things he ate, and his table was spread in whatever way, often without eggs or fish or any other fine thing. His drinking was not excessive, but it included a little wine and plenty of water.

On ordinary days he was awake for a large portion of the night, long before dawn. ${ }^{652}$ On Sundays, he was awake from the evening before Sunday until the morning. The priests took his vigils as a model because of the great love that he had for God and the greatness of his soul. And often he would not go back to bed for a long time, so that I even saw him nearly fall to the ground on numerous occasions because of the intensity of the practice.

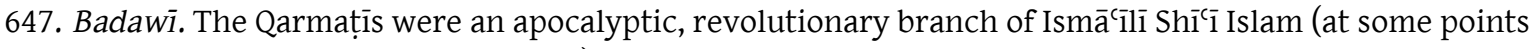
also closely associated with Zoroastrianism) that gained numerous adherents in Syria, among other regions, in the early fourth/tenth century. The movement lost much of its appeal, however, after the infamous, bloody Qarmați raid on Mecca during the hajj season of 317/930. At this point, numerous Qarmatīis entered the service of Sunnī rulers, including the Hamdānids. See Canard, Histoire, 1:315-18, 602-6, 632-34.

648. Ghazawāt.

649. For "Ṣārikha," Z reads: "Mārikha."

650. For "Halys," Z reads: "al-Sinn." As Canard notes, this is a slightly garbled reference to the Halys (Arabic "Alis"), now the Kizılırmak in northern Turkey. Șārikha appears in Byzantine and Muslim sources as well, and Canard writes that this text allows us to place it precisely on the Halys, "without doubt upstream from Sivas." Zayat, on the other hand, takes "al-Sinn" as the correct term and places it on the Tigris. The connection to Șārikha (as it appears in S, though it is misspelled in Z) makes Canard's reading more likely. See Canard, "Vie," 567; Zayat, "Vie," 26.

651. That is, Christ.

652. Fajr. 
We have mentioned a few things on this topic to show the difficulty of the life that he led, in which he had no prior experience. Anyone who has experienced this or has heard about it will surely know how difficult this path and practice were for a man who was raised in luxury.

\section{[5. The Catholicos Issue]}

It is now time to discuss what he did after his ordination. It was necessary to ordain bishops for the sees that were vacant. Which sees did he turn to first? He did not turn somewhere uncontested, nor to a see in which he had no history of opposition or favoritism or contention, but to the two sees that he had defended and opposed. His concern for them was not the same as it had been earlier, partial and headstrong. When he realized within himself that he had not acted in an appropriate way, as a result of $116 \mathrm{v}$ his favoritism and opposition, he decided to confront the aspects of the situation that he had previously ignored and to reform both his own intention and the things that were now under his control, even reforming his own beliefs.

Thus he approached the situation with a just balance, but also with wisdom and kindness. So he weighed the ordination on this balance and created two catholicoi: one for the City of Peace, his homeland, who was a man of Aleppo named David, ${ }^{653}$ and the other for Romagird (which he had opposed), a man of Antioch named Eutykhios. Who will not praise him for this kind and correct policy that led to peace and harmony? For he did not wrong his own city, but he also looked well upon the other, and he gave them harmony of souls and removed their discord and contention.

\section{[6. Other Vacant Sees]}

But did he then lie down on the issue of the other vacant sees? When he looked into the issue, did he consider bribes or intercessions, or a ruler's pleasure, or the terror of a powerful person's frown? No! On the contrary, when he saw that the delegation from a city requesting a bishop had mentioned someone worthy of ordination, and that person was pleasing to God and to him, he would lay his hands upon him immediately and confirm him, being pleased with what pleased them and yielding to their request. Or if he was not pleased with that person, he would choose someone else with whom they were also pleased, and would lay his hands upon him with the consent of the following ${ }^{654}$ synod. There was no delay, because the metropolitans who were before him could see that his mind was judicious, his determination was strong, no favoritism affected him, and he did not desire gifts or bribes. So how could they contradict him in anything he decided? ${ }^{655}$

653. For "David," Z reads: "Mājid." Tūmā Bītāar suggests that Mājid (if this is the correct reading) might be the author of a fourth-/tenth-century Arabic commentary on the Nicene Creed, though the evidence is limited, and the variant reading of S makes the identification even less likely. See Tūmā Bìțār, al-Qiddīsūn al-mansiyyūn fi al-turāth al-Anțākī (Duma, Lebanon: 'Ā'ilat al-Thālūth al-Quddūs, 1995), 385-86.

654. Lacuna in Z omits: "following."

655. S repeats: "his determination ... he decided." 


\section{[7. Church Administration]}

$117 \mathrm{r}$ He had a strong determination, a great heart, and a zeal to beautify and reform the church. He fought for it and defended it, never being moved or turned back. It should not be a problem for us to mention a story as proof. There was a priest, a physician, who committed a small fault, so the blessed father restrained him and suspended him from exercising the priesthood for some time. This priest was serving one of the Hamdānid emirs-a fierce tyrant obedient to no one, not even Ibn Hamdān. He asked him to intercede with the patriarch to secure his release and cancel his suspension. His intercession came without delay, because he believed that no one-not even one of the most powerful Muslims-would dare to disobey him. So how could the patriarch, a lowly Christian dhimmī? ${ }^{656}$

Therefore he addressed the patriarch gladly, saying, "Whatever sin was committed by my physician, the priest, transfer his offense to me, O patriarch, and forgive him." He answered him, saying, "That is not possible for me, O my lord the emir." He responded to him, saying, "O uncircumcised man, don't you fear me? Yet you dare to tell me 'That is not possible for me'? What could be impossible for you if I have commanded it?" The bold man responded to him, saying "Many things are impossible for me, $\mathrm{O}$ emir, if they relate to my religion, my doctrine, and my law. ${ }^{657}$ For we are in obedience to ${ }^{658}$ you, ${ }^{659}$ and in other things it is not possible for us $117 \mathrm{v}$ to disobey you. But as for what religion has forbidden, when it comes to these things we are prepared to face prison and the blades of swords." So he responded to him: "At least let me know what is this grave offense that has violated your religion." The disciple of Christ said, "Before this, O emir, the crime was only a little one, and it would be easy to make satisfaction for it. But now it is great, and it is undesirable to forgive it, because he asked you to intercede ${ }^{660}-$ you, a Muslim, who disagrees with us in doctrine! ${ }^{661}$-and the truth of this case is no secret, since the matter concerns only our church." The Hagarene answered him, saying, "From now on, be armed to the teeth, and know beyond a shadow of a doubt that you are going to die. I would take your head even if it were on the breast of the Great Emir."

What did this bold man do after that? Was he anxious? Did he relent? Did he bend the knee? Did he stop to send a message about it to Ibn Hamdān? Absolutely not! Rather, he considered all of that to be nothing more than scattered dust of no importance. He set out at once for Antioch and entrusted it to God, his defender. At that time he was in Aleppo. ${ }^{662}$ Praise God, his aim was not off the mark. And this was his zeal and ambition for all that would beautify and adorn the church.

656. For "because he . . Christian dhimmī," $\mathrm{Z}$ reads: "trusting in his kindliness, especially as he was a dhimmī."

657. Dīnī wa-madhhabī wa-nāmūsī.

658. For "are in obedience to," S reads: "do not obey."

659. Plural.

660. For "he asked you to intercede," Z reads: "I find it horrible."

661. Madhhab.

662. Z places "At that ... in Aleppo" after the word "Antioch" in the previous sentence. 


\section{[8. Care for the Poor]}

If the way of this virtuous man in these things was as we have described, did he behave the same way in other things-as one who does not bend over or yield, and not ${ }^{663}$ as the meek lover of humanity who lends his sympathy and inclines his ear to anyone who asks, anyone who needs his mercy and compassion? In other areas, who was stronger in compassion than Christopher, or more tender $118 \mathrm{r}$ in sympathy for the afflicted, or stronger in mercy for those in need, or more generous in giving to the pure? His wealth was not abundant, because he was working among the Gentiles, so his revenues were diminished. Nevertheless, as far as it was possible for him, he never cut short his generosity, support, care, and giving-not only once, but many times, and not only occasionally, but continuously-if it was possible, conditions were easy, and he did not face difficult obstacles.

He received messages, and he never failed to read them and to record with his own hand that one of the petitioners ${ }^{664}$ should be given documents, ${ }^{665}$ another clothing, another food, another drink, and still others similar things. I myself, the composer of this text, saw a priest who had told him a story, so he brought him into his presence and asked him about it, saying, "How many dependents do you have?" He answered him, saying such and such, so he said, "Let him be paid this much wheat, this much oil, and this much wine," enough to last him a year. When the priest was beginning to leave, he said to him, "Come back here. Where, $\mathrm{O}$ unfortunate one, will you get the price of milling ${ }^{666}$ or any seasoning? No; pay him this, too, whatever is enough for him." The saint did not let him leave until he had given him enough to satisfy him for the whole year.

Furthermore, whenever the story of an imprisoned or unjustly extorted person came to him, if it was possible to redeem them with small gifts, he did not hesitate to give and to redeem them from whatever was demanded of them. But if there was an intense difficulty, he never failed to ride over to the one making the demands, $118 \mathrm{v}$ asking them to forgive whatever they wanted the person to pay and to make any possible reduction to the sum. This was the image of a new Nicholas ${ }^{667}$ among us, passionate in concern for all who were in hardship and need. All of this is evidence of the things he would do of his own accord and of his generous kindness.

His head, his zenith, the prototype to which he adhered and whose likeness he boreChrist, the imitation of whom was always in his thoughts and whom he desperately wished to emulate-was not content to fill the bellies of thousands with a few pieces of bread, but added another satisfying gift with his two pure hands: he washed the feet of his disciples. ${ }^{668}$

663. Z adds: "How would he then have shown by his actions that he was a worthy disciple of Christ?"

664. For "petitioners," Z reads: "poor."

665. Waraq. Potentially paper money.

666. For "milling," Z reads: "flour."

667. A fourth-century CE bishop of Myra, famed for his generous gifts; inspiration for the modern Santa Claus and his counterparts. Nicholas was extremely popular in this period. See Roberts, Reason, 68-72, 105-8, 111.

668. Cf. John 13:1-20. 
So this disciple of his also strove ${ }^{669}$ not to be satisfied with what we have described and added another service with his two hands for those who were his brothers in their connection to his teacher. In Antioch there was an intense famine that naturally affected all the people. Therefore, Christopher gathered a group of those most severely injured by hunger-the elderly, the sick, priests, deacons, young people, and orphans. He gathered them, organized them into schools, and had them sit at tables while he stood, not satisfied just because they had abundant food, but sometimes taking the task into his own hands and serving them drinks, one by one. It is thus clear that he was a faithful slave of Christ, imitating him in a way that no other could match.

\section{[9. Educational Works]}

Since I have now mentioned young people and orphans, ${ }^{670}$ I must explain who these young people were. It is clear, $\mathrm{O}$ listener, that he extended his soul not only in love for the weak but also in excellence of mind and in the benevolent administration of everything for the greater good. He saw how tight and difficult things were and that for this reason people were falling behind in their learning-and that not only the poor and powerless but even the notables, the people of esteem, were failing in it. The holy Church of God was lacking, and most of the people had no $119 \mathrm{r}$ thought or care for learning.

So he thought of a major program that would show his good administration: he chose from among the powerful ${ }^{671}$ twelve young people, intelligent and distinguished, and handed them over to an insightful teacher, who would teach them the ecclesiastical sciences that surpass all others. Then he cast lots to choose other people from among the poor, especially orphans in bad situations -150 young people-and handed them over to three teachers who would teach each one whatever they could do skillfully. He ordered that three large kettles full of food should be cooked every day, each one taken to one of the schools, and that every young person should be given whatever was enough for them, along with whatever bread they needed. We must recognize how many benefits he provided here-namely, three major ones: first, food that the eater did not have to labor for; second, education without price or payment; third, noble service to the church ${ }^{672}$ These are all among his great deeds in this area.

\section{[10. Defending the Faith]}

He added to this an even greater help for the poor and support for the faith. The Hagarenes extract from all Christians in their countries a $\operatorname{tax}^{673}$ that we call the "head

669. Ijtahada.

670. S omits: "He gathered ... and orphans." This lengthy variant is likely an accidental omission in S due to the repetition of "young people and orphans."

671. For "powerful," Z reads: "wealthy."

672. Bī́a.

673. Jizya. 
tax" 674 but they call the "acquittal," 675 because it acquits those who pay it of any damages or prosecution. None of the Christians who live there can escape from paying it, because whoever does not pay is led without a choice into the religion of Islam. An amazing and lamentable spectacle can be seen there among the afflicted. Some Christians pay the acquittal with righteous intentions, if they can, and consider $119 \mathrm{v}$ it a charitable gift and a good deed, because it is something they pay on account of their religion. But other people are oppressed by it because they are impoverished. Thus the strongest $\mathrm{n}$ religion is not the one who hesitates to pay what they can, but rather the one who gives to assist the weaker person with whatever is demanded of them.

When Christopher, who held Christ within his breast, saw the harshness of these things, giving was not enough for him, and he was not satisfied with making additions to people's acquittal payments; rather, he went beyond that and paid from his own wealth the entire acquittal for people who could not pay. However, his wealth was wearing out, and it was not easy for him to do these things in the way that he believed he should, because he did not have abundant revenues. So how do you think he approached this issue? In precise imitation of John the Merciful, ${ }^{676}$ master of Alexandria. He happily asked the emir, Sayf al-Dawla, to help him in his love for the poor, and he did not reject him, because he was generous in nature and intensely favorable to the patriarch. He ordered the tax ${ }^{677}$ collectors to forgive 10,000 dirhams for him every year, and he ${ }^{678}$ would write messages to them on behalf of whomever he wished to help. So he might write to forgive the entire acquittal for one person and write for another forgiving half of the amount. Thus not a single Christian went over to the religion of Islam during his time.

\section{[11. Loyalty to Sayf al-Dawla]}

So the patriarch received-along with rewards from God-a strong welcome from Sayf al-Dawla the emir, and favor from the same Sayf al-Dawla. For this is part of human nature: not only does the one for whom good is done trust in the patronage of their benefactor, 120r but the benefactor also adds to the benefits given to them. The beneficiary derives benefits from the patronage, but then the benefactor wishes to give them still more benefits, going to great lengths in both quantity and duration-especially if they have goodness in their nature-so that their previous benefaction will not be made futile by their miserliness.

Thus when intense opposition and rebellion broke out against Sayf al-Dawla and persisted for a long time, the patriarch alone kept his distance from it and did not join the group of Antiochians who were rebelling against him. He did not even wish to speak to the insurgents.

674. Jizyat al-ru'ūs.

675. Barā’a.

676. John V, Chalcedonian patriarch of Alexandria 606-16 CE, known for his almsgiving.

677. S calls this tax kharāj; Z calls it barā’a.

678. That is, Christopher. 
Now, the reason for this rebellion was that the blessed Emperor Nikephoros ${ }^{679}$ was taking possession of the border country ${ }^{680}$ most of it by violence and war. Tarsus alone ${ }^{681}$ he took by a treaty of safe conduct, ${ }^{62}$ for hunger had ravished its people, and they had reached a disastrous state, inadequate for war. At the time, Sayf al-Dawla was suffering from paralysis ${ }^{683}$ in Mayyafariqin. ${ }^{684}$ As for the people of Tarsus, they came to the city of Antioch with their wives ${ }^{65}$ and children. The Hagarenes of Antioch were also concerned for themselves, so they went to Ibn al-Zayyāt, ${ }^{686}$ their governor, ${ }^{687}$ asking and begging him to stay with them and manage their affairs, since things were falling apart and becoming fragile. When Ibn al-Zayyāt saw that things were in such a state, he began to worry about Emperor Nikephoros. He loathed the idea and absolutely refused to stay.

The fear of the Antiochians increased at his refusal, which drove them to seek out Rashīq, ${ }^{688}$ Ibn al-Zayyāt's second-in-command. They asked him the same thing they had requested of the other, and he responded positively but indicated that they should submit to Emperor Nikephoros and yield to his rule. He reminded them $120 \mathrm{v}$ that this was the way of prosperity and that they would never attain the calm and tranquility that they desired if they did not obey him. They accepted his advice and sent messengers to the emperor, offering to bring money and to secure their agreement with pledges. Because the emperor was unyielding and was wary of them, he responded to the message they had sent, saying, "I do not accept money, because the emperor of the Romans has no need of it, and because the Muslims might give it today and refuse it tomorrow. Nor do I accept pledges, because while they have meaning for some people, most think nothing of them. I request only one thing, whenever you are ready and realize that it is an easy and insignificant thing for you to do: I wish to build on a rock formation within your city a fortress, in which I will have a stratēgos and a small number of others to defend you, and through them I will conquer."

When the Antiochians refused that, Raship felt ashamed and thought that he had become completely useless, so he decided to "uncover his head"-as the saying goes-in rebellion

679. Nikephoros II (r. 352-59/963-69).

680. Balad al-thaghr. Zayat translates this phrase as des villes du littoral ("the towns of the coast"), another potential (especially modern) meaning of thaghr, but it seems more likely that this is a reference to the Islamic geographical concept of al-thughür, the border fortresses on the frontiers of Muslim-ruled territory, especially on the Byzantine border.

681. For "took Tarsus alone," $\mathrm{Z}$ reads: "destroyed Tarsus and took it."

682. Amān.

683. Aflaja. The primary meaning of this word is "to be victorious," but the context (along with other historical sources) makes it clear that Sayf al-Dawla was suffering from paralysis, or fălij; see 'Izz al-Dīn b. al-Athīr, al-Kāmil fi al-tārīkh, ed. Muḥammad Yūsuf al-Daqqāq (Beirut: Dār al-Kutub al-'Ilmiyya, 1987), 7:279.

684. Modern Silvan, Turkey.

685. For "wives," S reads: "young people."

686. For "Ibn al-Zayyāt," Z reads: "Ibn al-Zamān." This variant continues throughout the text.

687. That is, the newly arrived governor of Tarsus; see Canard, Histoire, 648-49.

688. For "Rashīq," $Z$ reads: "Rashīq al-Nasīmī, who had come from Tarsus." This is the first of the edits that have been made to Z in order to bring it in line with the Dhayl of Yahyā b. Sa'īd al-Anțākī; see al-Anțākī, "Histoire," 797. 
against Sayf al-Dawla, especially because of his paralysis and inability to move, which made people think little of him. Once Rashīq had made this decision, ${ }^{689}$ he gathered his supporters together and headed for the city of Aleppo. ${ }^{690} \mathrm{He}$ took it by force without much effort and began to lay siege to the citadel, but it would not budge. A servant of Sayf al-Dawla named Qarghuwayh ${ }^{691}$ was there, and he would send out men every day to fight Rashīq. One of them-it was not clear who-struck him with a spear thrust during the battle, $121 \mathrm{r}$ so he died there, and when he fell dead, ${ }^{692}$ the others fled to Antioch. When they arrived, they were concerned for themselves, and they set a member of their group at their head as their emir. ${ }^{693}$ They remained firmly committed to their opposition and rebellion. The one who encouraged them in this was a person of Antioch named Ibn al-Ahwāzì, an intense and dynamic person who had been the manager of their affairs in the time of Rashiq. ${ }^{694}$

What did the patriarch do during this time of chaos in Antioch, which was only growing more and more difficult? His well-managed mind encouraged him to remain firm in the patronage of Sayf al-Dawla, so he withdrew to the monastery of St. Symeon of Aleppo. For even if Sayf al-Dawla was paralyzed at the time, his mind had not completely faded, and his tongue had not lost the power of speech. So while the patriarch ${ }^{695}$ was staying in the monastery, his situation was revealed by night to those in Aleppo, ${ }^{696}$ and it was unbearable for the rebel. He began to investigate the patriarch's connections and to arrest and harass his closest companions. He sealed up everything in the patriarchal cells ${ }^{697}$ and said aloud that if the patriarch did not come and take care of it, he would cause him even more grief.

Did the patriarch surrender to him, or get worried, or yield? No! He remained completely firm. One of his closest companions-Theodoulos, who became bishop of Seleucia after he

689. For "Once Rashīq . . . this decision," Z reads: "Then a man of Antioch known as al-Hasan al-Ahwāzī attached himself to Rashiq and took over the management of his affairs with the help of the people of Antioch. He was intense and dynamic, and he gave them hope that Sayf al-Dawla would never return to Syria (al-Shām). Dizbar al-Daylamī and a group of Daylamites who were with Qarghuwayh, the servant of Sayf al-Dawla, sought the protection of Rashīq. Rashīq and Ibn al-Ahwāzī set out." See al-Anțākī, "Histoire," 797-98.

690. Z adds: "Many battles took place between him and Qarghuwayh." See ibid., 798.

691. The spelling of this name is very uncertain. Zayat transliterates it as Qarghoyah and Canard (Histoire, 649-51) as Qargawaih.

692. For "lay siege ... fell dead," Z reads: "lay siege to the citadel for three months and ten days. Afterward Rashīq was killed by a spear thrust that hit him." Al-Anțākī includes the "three months and ten days" detail but not the detail about the spear thrust, which is probably a summarized form of S; see al-Anțākī, "Histoire," 798.

693. For "they were . . . their emir," Z reads: "they were concerned and afraid, and they made Dizbar al-Daylamī their emir and Ibn al-Ahwāzī his manager." See ibid.

694. For "The one ... of Rashīq," Z reads: "Qarghuwayh headed for Antioch and a battle took place between them, but Qarghuwayh fled and returned to Aleppo. Dizbar al-Daylamī went to Aleppo after him, but the companions of Qarghuwayh met him, fought him, and repulsed him, so he returned to Antioch." See ibid.

695. $\mathrm{Z}$ adds: "and those with him."

696. Z omits: "his situation ... in Aleppo."

697. $Z$ adds: "because of the inclination of the patriarch and those with him toward Sayf al-Dawla, which had been revealed to him." See al-Anțākī, "Histoire," 798. 
was killed and who built two beautiful churches ${ }^{698}$ in Antioch, for the Arkhistratēgos ${ }^{699}$ and for Chrysostom-observed him acting in this way. He grew bold and said to him, "Sir, when this is all over, do you intend to tell your church, ${ }^{700} \square 121 \mathrm{v}$ 'I am the good shepherd'? ${ }^{701}$ Do not leave your sheep for the ravishing wolves to snatch up! Consider the matter, then go and extend a hand to help them, and do not think about what the rebel wants, but about what is best for you and your flock." The patriarch said, "Hold your tongue and be silent, ${ }^{702}$ because you do not know what you are saying." So he had to hold his tongue.

After a little while, the servants of Sayf al-Dawla could no longer bear their shame quietly. They got him started on his journey and helped him get up, so he journeyed from Mayyafariqin to the area of Ma'arrat Mișrin, and war broke out between the two sides. The victory went to Sayf al-Dawla, and the chief officers of the rebels ${ }^{703}$ were taken prisoner. He put them in shackles and chains. ${ }^{704}$

Then the patriarch made his way to Aleppo, as happy as one who had triumphed in an agonizing $^{705}$ struggle. Sayf al-Dawla gave him the warmest welcome and he became his close companion in all things, a helpful and beloved intercessor. He went from the status of an insignificant follower to that of an influential friend, not to be accused or belittled, because in the time of hardship he had been faithful, constant, and patient in spite of his affliction. What he had given in the time of distress was repaid in the time of happiness with special treatment and preference. ${ }^{706}$

He saw those who had grieved him punished before his very eyes with beatings, abuse, and other types of torment that are impossible to bear, but he was not pleased as others were. He did not grow arrogant on account of his victory; rather, he mourned, he felt a tightness in his chest, his thoughts were troubled, and he said, "Have mercy, sir, and go easy

698. Haykalayn.

699. For "the Arkhistratēgos," S reads: al-aksīrātīqūs; Z reads: al-azkis 'üțus. I take this to be the Greek arkhistratēgos, "supreme commander" (a common epithet of Michael, the "supreme commander" of the heavenly forces), as suggested to me by Dmitry Morozov. Dick's edition has al-iksābtirīghūs, Greek hexapterygos, meaning "six-winged" (seraph). This must be Dick's guess at the original word, because it is not supported by either manuscript. The meaning is almost right, however, even if the word is not. See Dik, Sirra, 15, 46.

700. $B \overline{1} \mathfrak{c} a$.

701. John 10:11, 14

702. Lacuna in $\mathrm{Z}$ omits: "Consider the ... be silent."

703. For "the chief . . . the rebels," Z reads: "Dizbar and Ibn al-Ahwāzī and a large group of their soldiers." See al-Anțākī, "Histoire," 805. The term "rebels" translates khawārij (sg. khārijī), an allusion to the khārijī rebel group of the early Islamic period, but Ibrāhīm uses the term here in a general sense.

704. Z adds: "and brought them to Aleppo, and killed them, and he made his servant Taqi governor of Antioch." See ibid.

705. This word is omitted in Z and not fully legible in S. Dick's edition (Dīk, Sīra, 47) reads it as lūyā, but in S there seems to be at least one letter between the wāw and the yā'. I have read it here as law iyyan, although the meaning of “agonizing" works for Dick's reading as well. It could perhaps be emended to lūdīyā and read as a reference (via Greek and/or Syriac) to the gladiator games, Latin ludi.

706. $\mathrm{Z}$ adds: "because he was grateful to him for his act of distancing himself from those who rebelled against him, and so he preferred him and gave him special treatment." See al-Anțākī, "Histoire," 806. 
on those you have defeated, for the sake of $122 \mathrm{r}$ the one who helped you stand against them! Do not refuse intercession and forgiveness as much as your duty allows."

When he saw one of his neighbors, one of the Muslims of the city of Antioch, ${ }^{707}$ being punished and struck with far too many lashes, he did not sit patiently. He stood up, threw himself on the ground, and asked that the man's crime be given to him, and his request was not rejected. But what misfortune and evil grew out of this for him, I will explain in what follows. And that should not be surprising, even if it is improbable and strange. For so the nature of envy is imprinted on malicious souls, that their fire is ignited by goodness more than it is extinguished by kindness. When that envious one was set free and released from everything that had been imposed on him, he returned to Antioch without any consequences. If only it had not been so! ${ }^{708}$

\section{[12. Return to Antioch]}

After that, the patriarch stayed for a little while in Aleppo and then went to his city, bringing signed notes from Sayf al-Dawla to those whom he had dispatched to Antioch in an effort to get even with everyone who had helped the Antiochian rebels ${ }^{709}$ against him. He ordered them not only to absolve the patriarch and his companions of any responsibility but also to approve his requests-as often as possible-when he interceded on behalf of others, for the patriarch had taken up many such cases.

When he arrived in Antioch, he found a governor there, a servant of Sayf al-Dawla ${ }^{710}$ known as Taqi. ${ }^{711} \mathrm{He}$ also found the patrikios Kulayb ${ }^{712}$ seizing the wealth of the people, though he had not been commanded to take ${ }^{713}$ so much, $\quad 122 \mathrm{v}$ and confiscating all of their possessions. Sayf al-Dawla did not show them the kindness of restraining him from seizing their goods but acted out of pure self-interest, for he could see that these seizures were ${ }^{714}$ impoverishing the people, ruining the country, and eliminating any revenue. The fines had

707. It is not entirely clear, but it seems from the description in $§ 13$ that this may be Ibn Mānik, the future leader of the assassination plot (not actually named in S until §17).

708. $\mathrm{Z}$ adds: "Even so, there were many other elders of Antioch with whom Sayf al-Dawla was angry on account of their rebellion and whom he had arrested. The patriarch interceded with him for some of them and acted as his mediator with them, and he granted his request regarding them. So at that time, because they witnessed his powerful position with Sayf al-Dawla, their souls became set in envy and resentment of him." See al-Anțākī, "Histoire," 806-7.

709. Khārijiyyinn, another allusion to the early Islamic khawārij; see note 104 above.

710. Z omits: "a servant of Sayf al-Dawla."

711. For “Taqī,” Z reads: “Taqī al-Dīn, whom we have mentioned.” See al-Anțākī, “Histoire,” 805.

712. Kulayb, likely a Syriac Orthodox Christian, later became basilikos of Antioch and eventually of Melitene under Byzantine rule; see ibid., 2:369, 373-74, 420. Al-Anțākī simply calls him a "Christian” (nașrānī), but Michael the Syrian mentions that he sponsored construction work at a Syriac Orthodox monastery in Melitene; see Michael the Syrian, Chronique, ed. and trans. J.-B. Chabot (Brussels: Culture et Civilisation, 1963), 3:126, 4:553; Catherine Holmes, "'How the East Was Won' in the Reign of Basil II," in Eastern Approaches to Byzantium, ed. Antony Eastmond, 41-56 (Aldershot: Ashgate, 2001), 49.

713. Z omits: "the wealth ... to take."

714. Z omits: "but acted ... seizures were." 
brought people to such a state that they barely had enough to pay for housing, taxes, and other such things. When the patriarch saw the people of Antioch struggling under these demands, whom did he not deliver and redeem from the bulk of their fines? Whose burden did he not lighten? Whom did he not redeem entirely, if he could?

Now, there was a resident ${ }^{715}$ of Antioch who was very wealthy because he owned extensive real estate and had many sources of income but who thought of nothing but himself. He would hunch over as he walked, never raising his head from the ground, because he was such an arrogant miser. The wretch had never used a bit of his money to help anyone, not even himself. So the companions of Sayf al-Dawla seized him and began to punish him, demanding three and a half qințārs ${ }^{716}$ of gold. He sent to the holy patriarch in the midst of his punishment and said to him, "Have mercy, sir, on a wretch like me." By my life, the patriarch got to work quickly, as usual, eager to help anyone who asked for his aid. He rode over at that very moment, relieved him of his torment, and mediated for him regarding the amount that was demanded. He did not stop making requests on his behalf until he had reduced the massive sum from 25,000 $123 \mathrm{r}$ dinars to a tenth of that, that is, 2,500 dinars. The tax collectors said, "He will not pay even this amount without being punished and beaten." So the disciple of Christ, who resembled him in every way, said, "I am asking you not to go overboard by punishing him any more, but to hand him over to me, and I will be responsible for the whole sum." He was handed over to the patriarch, and the tax collectors would later demand from the patriarch the entire sum, and then the patriarch would have to ${ }^{717}$ demand it from him. Whenever the patriarch would write to him about this, it was like his heart was being pierced, as the arrows of envy worked within him. Thus he later consented to kill the one who had been his benefactor.

\section{[13. The Assassination Plot]}

Now the killing of the patriarch-or rather, his martyrdom-was carried out by the decision of this man and others. It was organized in secret and proceeded little by little. For because Sayf al-Dawla was inclined toward him, ${ }^{718}$ the raw material of envy had accumulated in the hearts of the Muslims. However, they were not all agreed on killing him. On the other hand, those who did agree were among those whom he had most generously benefited, for the father of envy was at work within them.

The proof was as follows: after Sayf al-Dawla died, ${ }^{719}$ people came from the land of Khorasan, warriors hurrying on their way to Antioch. ${ }^{720}$ That cursed one, whose benefactor

715. It is not entirely clear, but it seems from his description in $\S 13$ that this may be Ibn Mahmūd.

716. From Latin centenarius/Greek kentēnarion, the qințār is a variable weight equivalent to 100 arțāl and sometimes used to refer to an indeterminate (large) amount. Based on the typical Syrian qințār of $256 \mathrm{~kg}$, the amount demanded from this miser was likely almost $900 \mathrm{~kg}$ (nearly one ton) of gold.

717. S omits: "the entire ... have to."

718. That is, Christopher.

719. 25 Șafar 356/9 February 967.

720. For "from the ... to Antioch," $\mathrm{Z}$ reads: "from the land of Khorasan, intending to raid the Romans, so they traveled to Antioch, and its people gave them the most beautiful welcome." See al-Anțākī, "Histoire," 807. 
the patriarch had been, ${ }^{721}$ in whom the venom of envy was at work, and who had thought to plot against the patriarch because of his empowerment, was afraid. If he did not communicate with the leader of the Khorasanis, ${ }^{722}$ he would miss the chance of killing him.

So he summoned ${ }^{723}$ Ibn Mahmūd, for whom $123 \mathrm{v}$ the patriarch had vouched, ${ }^{724}$ who had made known to him what he had against him in his soul; and he summoned another person, a neighbor of the patriarch called Ibn Di'āma. He said to them, "What do you say? Do you think we should leave this infidel alive until a governor arrives, so that he will be sitting in court while we are punished, as we were in the time of Sayf al-Dawla?" They said, "That is not right." So he said, "Then what do you think about requesting a written fatwa $\bar{a}^{725}$ about him from the jurist?" They praised this idea and with one mind, they all wrote a note called a fatwa. ${ }^{726}$ They did not mention the patriarch by name but said, "What is your opinion, 0 jurist, on the just response to someone who plots against a Muslim fortress?" He answered that the person must be killed.

The leader of the plot said to the other two, "This fatwā is the way: if we show it to the masses, they will kill him immediately." This was not actually his opinion; rather, because he was also a Persian and knew the language of the Khorasanis, he was secretly planning to call a group of the soldiers who had been sent to Antioch and show them the fatwa so that they would kill the patriarch without hesitation. This helped to convince them and to spur them toward what he wanted.

\section{[14. The Patriarch's Decision]}

One of the patriarch's neighbors, a prominent Muslim named Ibn Abī 'Amr who was a true friend and adviser to him, found out about this. So he rushed over and said to him, "What are you doing? Get up quickly and look out for yourself! Otherwise, you should be aware that you are going to be killed soon." He said, "Why? For what reason?" He said, "Because $124 \mathrm{r}$ a malicious group gathered against you and consulted the law, and the jurist gave a fatwā that says you must be killed." He asked for more information and said, "What do you think I should do?" He said, "At the moment you are not under arrest, so leave through the city gate at the end of the day, and when morning breaks, you will be

These soldiers were likely coming to reinforce the frontier defenses and preserve Muslim control of Antioch as word spread of Byzantine advances in the region; see Kennedy, Prophet, 238, 240.

721. For "whose benefactor ... had been," Z reads: "whom the patriarch had taken under his wing." This is Ibn Mānik, likely the man whose punishment he alleviated at the end of §11 above.

722. This leader is not named here, but al-Anțākì ("Histoire," 807) claims that his name was Muhammad b. 'Īsā.

723. $\mathrm{Z}$ adds: "three of the elders and model citizens of Antioch for whom the patriarch had mediated and interceded." See ibid.

724. Z omits: "for whom ... had vouched."

725. Throughout this passage, both $\mathrm{S}$ and $\mathrm{Z}$ use a variety of spellings for this word, including fatwa (in Z), fita (in S), and futya (in both).

726. Normally, fatwā would refer more specifically to the jurist's response to their question, but Ibrāhīm apparently uses it to mean both the question and the answer. 
near Aleppo, and no one will follow you. That is what I think." He said, "Sir, may God reward you with good things. As for me, I must think about what I will do."

The man departed, and the patriarch summoned that man who had said to him when he was at the monastery of St. Symeon, "Why do you not go to Antioch in the time of rebellion? Are you, sir, the sort of person who can say after this, 'I am the good shepherd'?"727 When he arrived, he ${ }^{728}$ said to him, "You should know, O man, that our neighbor so-and-so came to me this very hour and told me such-and-such. What do you think?" The man said to him, "What could be better than this, sir? Ask God's blessing and do it!" He said, "If I do this, $\mathrm{O}$ insolent one, you will be among those who mock me afterward, saying, "Can you say tomorrow, "I am the good shepherd"?" The man answered, saying, "Sir, that was different." So the patriarch said to him, "Yes, it is not hidden from me that it was different. ${ }^{729}$ That is why, because I did not agree with you then, I did not do it. And when I did not do it, even you knew that none of the Christians would be harmed as a result, because no one was demanding my murder at that $124 \mathrm{v}$ time. But now, my murder is demanded, and not simply demanded, but demanded with intense desire and effort. For those who demand my murder are envious, and the venom of envy is concealed within their bodies. So if I slip out of their hands and they cannot inject their venom into me, they will not leave behind a single Christian or a single church. This is the time, O man, when I must say not only 'I am the good shepherd' but also that 'the good shepherd lays down his life for his sheep. ${ }^{730}$ Soon you will see this beard dyed with my blood." With that, he moved his hand toward it. His speech ended with this saying, and after that he said not another word; he was almost ashamed at the thought.

\section{[15. The Assassination]}

Afterward, he gave it some thought and decided to meet with that evil man who was preparing to act against him. He knew beyond a shadow of a doubt that he had mixed for him the brew ${ }^{731}$ of death. However, he did not think that he would do this in his home, for no desert Arab, ${ }^{732}$ nor any esteemed person among the Muslims, would consider this a good way to commit a crime. So after a little while, he sent him a note, showing neither wariness nor fear: "Sir, I would like to meet with you. Please tell me what you think and I will be available to come to you." ${ }^{733}$ He saw this message as an opportunity and as potential spoils,

727. See $§ 11$. The man’s name is Theodoulos.

728. Christopher.

729. S repeats: "So the ... was different."

730. John 10:11.

731. Qahwa. This Arabic word became the word "coffee," and it is the ultimate source of the beverage's name in all languages, including English. However, coffee was (most likely) discovered several centuries after the composition of this text. At this time, according to Lisān al-'Arab, qahwa referred to a type of wine. In any case, the metaphorical meaning of the phrase is clear.

732. A'rābì.

733. Z adds: "When Ibn Mānik heard this." See al-Anțākī, "Histoire," 808. 
and he said, "I am not available at the moment, but later I will send for you." Then he spent his entire day on the plot and did not send him anything because he was busy deceiving him and strengthening what he had cooking for him.

When $125 \mathrm{r}$ much of the night had come and gone, and the patriarch had eaten his customary food, he ${ }^{734}$ sent to him, saying, "I am now available, O patriarch, so if you want to come, then come." When the lamb of Christ heard this message, at such an unusual time, he was stunned and said to his companions, "What is the right thing to do, O people, when we are facing two irreconcilable options? Going at this time of night is neither proper nor appropriate, because the food is already in my mouth, and my strength-I am too weak to speak at this point. But postponing would also lead to trouble, because then we would be the ones who missed the appointment. But there is a way: can one of you check my breath? If you smell wine on me, we will use that as an excuse and say that it is not possible at this time. But if my breath is clean, we will go anyway." When one of them checked his breath and there was no odor, ${ }^{735}$ he went on his own two feet, like a lamb going to the slaughter ${ }^{736}$ of its own free will.

That cursed one welcomed him and greeted him with affection, even as deceit was concealed within his heart. When they sat together, he was full of wrath and hatred against him, so he could not wait for the patriarch to begin speaking, but said to him, "What are you thinking, O patriarch? You are one of the people of this town, dwelling among us, yet you think badly of our interactions and act against us." The patriarch said, "And how is that, sir? What do you mean?" He said in response, "Because you correspond with the emperor of the Romans, ${ }^{737}$ and you correspond with the servant of Ibn Hamdān." So he said, ${ }_{125 \mathrm{v}}$ "And what is the evidence for that, sir? Who has found such a letter from me?" $\mathrm{He}^{738}$ got up as if he were searching for a letter, then suddenly stood and spoke in Persian, summoning the Khorasanis whom he had prepared for the murder.

Still, he was worried and trembling. Not only was he a coward, but to both heart and eye, he looked like a bat. For he was hardly taller than a span, ${ }^{739}$ only by a little. He looked like a bat in color, facial expressions, and complexion. When those whom he had prepared and summoned arrived, he said to them in their language, his teeth chattering, "This is the one you are looking for! This is the man who wants to hand over this city! This is the enemy of the Muslims! Here you are, and here he is! Cut him to pieces without pity!"

If he were the enemy of the Muslims, ${ }^{740}$ you mouse's eye, you complete rat, then why was he not your enemy in the time of difficulty? Rather, he was your friend, the friend who

734. Ibn Mānik. Z calls him "that cursed one."

735. S repeats: "and there was no odor."

736. Cf. Isaiah 53:7; Jeremiah 11:19; Acts 8:32.

737. $\mathrm{Z}$ adds: "and incite them to come toward us, and encourage them against us." See al-Anțākī, "Histoire," 809.

738. For "He," Z reads: "Ibn Mānik." See ibid.

739. A span (Arabic shibr) is the distance from the end of the thumb to the end of the little finger, roughly $23 \mathrm{~cm}$-obviously hyperbole in this case.

740. Lacuna in Z omits: "Here you ... the Muslims." 
was helpful to you and delivered you from torment! But when this one whose soul was like that of a crocodile, requiting goodness with evil, gave the command regarding him, the sacrificial lamb of Christ stretched out his neck without any alarm, waiting for his head to be taken.

Those people had not swords, but long daggers. One of them made him stand up while the other struck him with a long dagger, and all of the iron passed through his belly. The sword of $\mathrm{God}^{741}$ was sharpened, but at that time it was held back, as God's judgments often are, awaiting punishment at another time. When the martyr fell to the ground, his head was first cut off, then his pure body was dragged around on a ladder by the command of the one who had rejected both God and goodness. It was immediately thrown $126 \mathrm{r}$ into the river after the gate was opened for it at night. ${ }^{742}$

But this could not be hidden from the Christian populace, and when their shepherd fell, they were stunned, struck by what had happened so suddenly, and intensely anxious. They scattered into the houses of some of the Muslims, where they hid. However, no one sought them out, for it was necessary that the earlier saying of their father should be fulfilled: "If they satisfy their thirst for revenge by killing me, and inject into me the venom concealed within their hearts, they will not pursue anyone but me."

\section{[16. Plundering the Church]}

As for that animal with the ferocious appearance, ever increasing in beastliness, he kept quiet for most of the night, because he was on the edge of losing his mind for good. But when dawn had come, he returned to himself and directed his guards toward the church $^{743}$ and the patriarchal cells. There was a group to search each of the two places. ${ }^{744}$ In the patriarchal cells, they found nothing but some foodstuffs, such as wheat, figs, and oil, preserved as provisions for the church and nourishment for the brothers in Christ. How could they find anything else when there was no gold ${ }^{745}$ hoarded there? How could he hoard when his expenses exceeded what he took in? Nor was there any clothing. How could any belong to someone whose clothing was only wool ${ }^{746}$ without even a monastic habit? ${ }^{747}$ But there were a few priestly funeral garments there that belonged to his predecessors throughout time, and they took all of them.

And they opened the treasury of the church, and when they did not see anything there, they punished the treasurer until he showed them the hidden gold and silver utensils of the

741. Sayf Allāh. In S, the scribe mistakenly wrote Sayf al-Dawla before crossing out al-Dawla and writing Allāh.

742. For "then his ... at night," Z reads: "and thrown into the furnace of the bath in the neighborhood of Ibn Mānik's house. Then his pure body was immediately brought out of the city gate by night and thrown into the river." See al-Anțākī, "Histoire,” 809.

743. Bīca. Z adds: "the Church of Cassian" (Kanīsat Qusyān). See ibid., 809-10.

744. Lacuna in Z omits: "to search ... two places."

745. Z adds: "or silver."

746. $S \bar{u} f$.

747. Askīm rahbāniyya. Askīm is from the Greek skhēma. 
church, $126 \mathrm{v}$ along with the silk garments that were kept to decorate the church. They left nothing but a few copper things and manuscripts. ${ }^{78}$ Out of all this, the cursed one did not give those who had shed the pure and innocent blood ${ }^{749}$ everything that they had agreed. He kept back some of it in order to give it as a gift to the Persians whom he was expecting to come on their way to the land of the Romans. In the midst of all this, they arrived, and the Antiochians welcomed them magnificently. That cursed infidel was especially important for them, not only because of what he brought them, but also because he shared their tribe and language. And they began to raid the Roman border regions ${ }^{750}$ and to devastate them.

\section{[17. Divine Retribution]}

Now the blessed Emperor Nikephoros was busy with the Bulgars. When he returned, he sent Peter the Stratopedarkhēs, ${ }^{751}$ who had a battle with the Khorasanis near the city of Alexander known as Alexandria, ${ }^{752}$ and the Persians were put to flight after extensive fighting. ${ }^{753}$ With them was a group of Muslim assassins of Tarsus ${ }^{754}$ who had advised them not to fight, nor to keep making war, for they said, "The army that is fighting you is an imperial army, and you have no power against them." The Persians did not accept this but persisted until their bravest men and most of the others were killed, and their chiefs were taken captive.

All of this was a necessary judgment from Heaven, for justice could not delay in avenging the blood of the martyr. Here it seems that we may go on for too long-even if it would be insufficient, coming from us-in clarifying the marvelous and efficient system, working according to an uninterrupted arrangement, by which the wicked received an evil repayment for their deeds. But telling the story contains some $127 \mathrm{r}$ benefit, for from these facts everyone can verify what prophecy tells us about falling into the hands of the living God: both that it is terrifying, and that repayment might come quickly, or else only a part of it might come quickly, in which case it is evidence that the remainder will later come to pass.

748. $\mathrm{Z}$ adds: "And they also took the chair of St. Peter the Apostle, which was made of palm wood overlaid with silver. They kept it in the house of one of their elders, known as Ibn 'Āmir, and it remained in his house until the Romans took possession of the city." See al-Anțākī, "Histoire," 810.

749. Z adds: "those of his nation."

750. S omits: "and language ... border regions."

751. An army commander. S: al-isțirātūus.

752. This is Alexandretta, now known as İskenderun.

753. For "and the ... extensive fighting," Z reads: "which is between Mopsuestia and Antioch, as they were returning from their raids. He attacked them, killed their bravest men, and took the sālār of the army and others as captives." See al-Anțākī, "Histoire," 814.

754. For “Tarsus," Z reads: “Ṭarțūs." 
One of the chiefs of the Hagarenes ${ }^{755}$ imprisoned in that war was the sālār $r^{756}$ of the army. The Antiochians purchased him for a massive amount of money, garments, and prisoners ${ }^{757}$ who were in Antioch and whom the people had taken captive. ${ }^{758}$ When it was done, ${ }^{759}$ he came to Antioch, and some of its people welcomed him and celebrated his arrival. However, as much as he entered in honor, and they honored and exalted him beyond any legitimate level, in the end he had the most shameful and disgraceful exit. For the Antiochians could not endure what they were undergoing at the hands of his men who had escaped the fighting. They had returned naked, and he like them. Thus necessity forced them to seize whatever would preserve them, and their hands stretched toward the wealth of the Antiochians. ${ }^{760}$ They did not endure that from them, but resisted them, war broke out between them, and the people of the city drove them out naked.

The Antiochians who fought them were in two bands, and the chief of one turned against the chief of the other and killed him. For someone had come from Egypt ${ }^{761}$ one of the brigands ${ }^{762}$ of Tarsus ${ }^{763}$ who had fled. He returned with a small band to raid the Roman border region. ${ }^{764}$ The chief of the other band was a Kurd from Būqa $\bar{a}^{765}$ called 'Allūsh, and the one who came from Egypt was a Black man named al-Rughayli. ${ }^{766}{ }_{127 \mathrm{v}}$ At that point the city was in the hand of 'Allūsh. ${ }^{767}$ Al-Rughaylī entered and greeted him, and when he bent over to take his hand, his sword was sideways on his knees. So al-Rughayli drew it, struck him with it, and killed him. Thus he dared to do two impressive things at one time and openly: drawing a chief's sword from his lap, and quickly killing him without a pause. So the followers of the one who was killed were scattered, even though they were many, and command passed to the killer, even though his men were very few. ${ }^{768}$

However, his rule did not endure and his time was not long, for Peter the Stratopedarkhēs came shortly with a huge contingent from the army of the praiseworthy Emperor

755. Z reads: “Khorasanis." See al-Anțākī, "Histoire,” 814.

756. A Persian word for a chieftain or leader.

757. $\mathrm{Z}$ adds: "whom they had previously taken captive from the Romans." See al-Anțākī, "Histoire," 814.

758. Z omits: "whom the ... taken captive."

759. For "When it was done," Z reads: "When the salār was set free." See al-Anțākī, "Histoire," 814.

760. For "and their ... the Antiochians," Z reads: "so they overpowered the Antiochians and began to seize their wealth and their goods." See ibid.

761. For "For someone .. . from Egypt," Z reads: “A Black man had come from Egypt to Antioch.” See ibid., 822.

762. "Brigands" translates șa ălīk. My impression is that this man had escaped from Tarsus during the Byzantine conquest.

763. $Z$ adds: "known as al-Rughaylī." See al-Anțākī, "Histoire," 822.

764. Z adds: "This was the chief of the first band."

765. Often spelled Būqa, a fortress and village near Antioch, somewhat important in the early centuries of Islam but eventually lost.

766. Z omits: "and the ... al-Rughayli." As with many of the names in this text, the correct spelling of the name "al-Rughaylī" is uncertain (in Arabic as well as in English).

767. For "in the hand of 'Allūsh," Z reads: "in his hand."

768. $\mathrm{Z}$ adds: "and al-Rughaylī took control of Antioch." See al-Anțākī, "Histoire," 822. 
Nikephoros. When he came, he conquered the great city that very night, ${ }^{769}$ although he had not even been seeking it. He found it weak because of the previous raids on its territories, and he found its people neglecting to preserve and protect it, because they had not heard the news that someone was coming for it. They could not in one moment gather enough men to climb the mountain and defend the wall, ${ }^{770}$ so the Romans quickly climbed it. They found it empty, so they were able to climb it. They took possession of the city ${ }^{771}$ and took everyone in it captive, and no one escaped except that cursed one who was the foundation of the whole catastrophe. ${ }^{772}$

Now see here, $\mathrm{O}$ listener, how the just judgment for earlier actions is delayed but comes to you more perfectly later on. For he had gone out of the city at night, seeking to find safety in some place within the lands of Islam, and at first the affair remained hidden. He was happy, not only because $128 \mathrm{r}$ he alone was saved from captivity, but also because he was able-as he thought-to trick, deceive, and lie to God and the angels, so that they had carried him through the air and saved him. This had long been his habit, and he had achieved precision in the art of tricking, swindling, and lying to the Lord of the Worlds. ${ }^{773}$ However, just judgment got ahead of him, and on the road, a bāqū $\bar{a}^{774}$ of Syrians met him on Jabal al-Aqrac ${ }^{775}$ They did not know that the city had been conquered. ${ }^{776}$ They seized him and brought him there once they found out about the conquest from the people who were with him. At that time the stratopedarkhēs was in Aleppo, and its people were seeking

769. For "he conquered . . very night," Z reads: "Michael Bourtzēs, who was in charge of the fortress of Baghrās, joined him, and they continuously besieged the great city." See ibid.

770. Antioch lies at the base of Mount Silpius, and its historic city walls climb up and enclose a portion of the mountain to provide additional protection from higher ground.

771. Z adds: "on Thursday, when thirteen nights had passed from Dhū al-Hijja, in the year 358 [28 October 969]. The Muslims threw fire to turn the Romans away from them and opened the Sea Gate, and some people left through it." See al-Anțākī, "Histoire," 822-23.

772. Z adds: "Ibn Mānik." See ibid., 823.

773. $R a b b$ al-'`̄amìn.

774. S reads: rāqūla, here and below. $\mathrm{Z}$ adds: "that is, a gang" ('așaba). This seems to be an obscure Syriac word, mentioned only in Thomas Audo's dictionary: the agent-noun form of the equally obscure verb bqal, usually used for plants, meaning "to sprout, blossom, shoot up." The literal translation would thus be "one who shoots up" or "springs up," perhaps indicating that these Syrians were a band of outlaws "springing up" in rebellion against the various rulers of Aleppo, Antioch, and beyond. Such outlaws would no doubt find the mountains a congenial site for their operations. Another possibility is that this is the Greek word bakyla, meaning "sticks" or even "fasces," or the Syriac būqālā (cowherd). Could there be a connection to the Qarmați rebels known as Baqliyya, the "green vegetable people," thanks to their ascetic vegetarian diet? This is not impossible, especially given the presence of Qarmatīs in other parts of the Life, but mentions of the Baqliyya are mostly confined to the Sawād region of southern Iraq, and it would be strange to hear of Qarmațīs who are also Suryān (and thus likely Christian). Canard ("Vie," 565) even suggests that this may be the name of a tribe. If $r \bar{a} q \bar{u} l a$ is the correct form, the Arabic word rāqūl, referring to a type of rope, is a possible origin, along with the Syriac rakālā (peddler, merchant). The derivation of the present meaning is unclear in any case, and it is no surprise that the scribe of $\mathrm{Z}$-or one of his predecessors-felt the need to insert a less obscure Arabic gloss.

775. Also known as Mount Kasios, now on the border between Turkey and Syria, just south of Antioch. Z adds: "They used to raid Antioch." See al-Anțākī, "Histoire," 823.

776. Z omits: "They did ... been conquered." 
a peace settlement with him. They agreed on a massive amount of money and brought it to him, and after he had checked the amount, he returned. So the people of the bāqūla brought him that cursed one, whom they had taken captive; he was known as Ibn Mānik. ${ }^{777}$ He handed him over to someone who would guard him until he could determine what he needed to do with him. ${ }^{778}$

The chiefs of the army gathered to deliberate on the issue, ${ }^{779}$ and some of the chiefs advised that he should be brought to the emperor. But others disagreed and said, "Who is this man that he is worthy to be brought into the imperial presence? The most appropriate thing is to kill him, in order to avenge the innocent blood that he shed." But the others, who were advising that he be brought there, answered them and said, "It is not right to pollute that pure blood with this impure blood." At last the patrikios Eustathios, strategos of Cappadocia, known as Maleinos, said, "Is the patriarch here ordering us to kill him, so that his blood can be 'polluted,' as you say? He completed what was necessary for him to do, $128 \mathrm{v}$ then departed and arrived at a place where he awaits a beautiful reward from God. But as for us, if we are Christians, then we must avenge the injustice that was done to him."

When he said something like this, it convinced them, and they sent the one who had been judged to the bridge at the Sea Gate, from which he himself had thrown the noble body into the river. He was cut apart with swords, piece by piece, but the pieces were not thrown into the river, for he was not worthy of that. Rather, each one was thrown at random onto the ground, and they became food for the birds and the dogs. As for the other two ${ }^{780}$ who had shared with him in spilling the blood, they were sent to the prison of Tarsus. ${ }^{781}$ They were not sought at that time, and no judgment was carried out in their cases. ${ }^{782}$

\section{[18. The Remains of the Saint]}

Now as it happened, the emperor ${ }^{783}$ was killed before learning the news from Antioch, and the empire passed to the son of Tzimiskēs. ${ }^{784}$ He quickly sent Theodore, ${ }^{785}$ a monk from Koloneia, and made him patriarch of the City of God, Antioch. He arrived here with those who brought him and immediately began to inquire about the story of the martyr and to seek out the remains of his pure body. For his body had appeared eight days after his martyrdom, which was on the night of the twenty-third day of May. ${ }^{786}$ It appeared on an

\footnotetext{
777. For "They seized ... Ibn Mānik," Z reads: "They seized him and brought him to the stratopedarkhēs."

778. $\mathrm{Z}$ adds: "And he bestowed massive favors on the people of the bāqūlā."

779. Z omits: "The chiefs ... the issue."

780. For "the other two," Z reads: "Ibn Maḥmūd and Ibn Dicāma."

781. $\mathrm{Z}$ adds: "and remained there a long time." See al-Anțākī, "Histoire," 825.

782. Z omits: "and no ... their cases."

783. $\mathrm{Z}$ adds: "Nikephoros."

784. John I (r. 359-65/969-76).

785. Theodore II (bishop 359-65/970-76).

786. Z adds: "in the year 356 of the hijra." This year corresponds to 967 CE. See al-Anțākī, “Histoire,” 809.
} 
island in the river, where it was caught on an oak tree. ${ }^{787}$ The noble head was not with it, and it was said that the infidel had burned it. Some Christians of Antioch went out secretly and buried it in the holy monastery known as Arshāyā. ${ }^{788}$ When Patriarch Theodore learned this, he did not delay in sending for what was $129 \mathrm{r}$ there but went to the holy monastery and took up the relics ${ }^{789}$ of the saint with the pure clergy and a crowd of believers. They went before them to the city with a procession ${ }^{790}$ and a great assembly and put them in a fine marble sarcophagus, which they placed on a marble table in the western part of the Great Church. ${ }^{791}$

After a few years, Abba ${ }^{792}$ Nicholas the patriarch ${ }^{793}$ again moved him with honor and put him inside the house of St. Peter the Head of the Apostles, with his staff, ${ }^{794}$ his chair, numerous balms, ${ }^{795}$ the relics ${ }^{796}$ of Patriarch Babylas and Patriarch Ignatius, and other things: the balms of St. John the Baptist, the honorable lance, the staff of Chrysostom, the belt of St. Symeon the Stylite of Aleppo, and so on. All of them are in the treasury of St. Peter to this day.

\section{[19. More Divine Retribution]}

However, the highest judgment of those who shared in the killing did not delay long after that. One of the two, Ibn Mahmūd, was in the prison of Tarsus in total misery, distress, and pain of soul and body. He was punished for his deeds until he handed over his miserable soul, which had rejected goodness and repaid its benefactor with a vileness that cannot be surpassed. The other, Ibn Dicāma, remained in prison until the just hand of the patrikios ${ }^{797}$ Bourtzēs came to Antioch. He sent people to take him, weigh him down with a stone, and throw him into the river. So the three-Ibn Mānik, Ibn Maḥmūd, and Ibn Di āma, who had taken upon themselves the murder of the saint-received the recompense for their actions, and in the afterlife they await eternal punishment.

787. Tarrāsh, from Syriac țarāshā.

788. Located just outside Antioch. See Nasrallah, “Auteurs," 85; Claude Cahen, La Syrie du Nord à l'époque des Croisades et le principauté franque d'Antioche (Paris: P. Geuthner, 1940), 324.

789. For "relics," Z reads: "body." S has limsanā, which I take to be a garbled version of the Greek leipsana, "relics."

790. Lìtīn. Zayat and Dick both interpret this word as a transliteration of the Greek litēn (Zayat simply writes it in his French translation as $\lambda_{\imath \imath \eta}$ ), meaning "procession." I see no preferable alternative translation for this enigmatic word, though as Canard notes ("Vie," 569), "one would like to find there a note and other examples."

791. This seems to be the Church of Cassian mentioned earlier.

792. For "Abba," Z reads: "Saint."

793. Nicholas II (bishop 415-21/1025-30).

794. Shabūqa, from the Syriac shabūqtā.

795. Huyūl. As Canard notes ("Vie," 568), this is a technical term for a "miraculous liquid that oozes from certain icons," as described by Zayat in his history of Șaydnāyā; see Habib Zayat, Khabāyā al-zawāyā fi tārīkh Șaydnāyā (Harissa, Lebanon: Imprimerie de Saint Paul, 1932), 144-51.

796. Libsānāt, from Greek leipsana. Z reads: "clothing” (libāsāt).

797. Z adds: "Michael." See al-Anțākī, “Histoire," 825. 


\section{[20. Comparison with Other Saints]}

As for $129 \mathrm{v}$ the saint, he has reached the dwellings of the priests in the highest heavens, mingling with the righteous, standing with the ascetic monks in the ranks of the earlier martyrs. For he closely followed and tightly adhered to one of the saints; another he contended with-neither far from the target nor far behind-and another he left behind and surpassed, as is obvious from his actions and the well-known evidence of his deeds.

For ${ }^{798}$ Abraham left his homeland by the command of God and became the father of the nations he was promised. Did this man not also set out of his own accord and give birth to many spiritual children? And Isaac became engaged to Rebekah by correspondence, ${ }^{799}$ but this man became engaged to the holy church directly. And Jacob inherited the blessing of his father, but by a trick-he inherited it as a son, but to be precise, he took the blessing itself by a trick. ${ }^{800}$ And as for his two wives, he took them only after labor and misery on their account, all the while looking toward an outward reward that he would receive. ${ }^{801}$ But this man, without fraud and in complete truthfulness, received blessings from the mouths of many, and while he was not lacking in labor for the sake of his spiritual bride, ${ }^{802}$ he was not looking toward a present reward but was ensuring that he would receive the unseen, anticipated reward.

As for the gentleness of David, by my life, he did not possess it completely, for a small remnant of the vanity of the secretaries remained within him. The right way to approach this topic is to say that even if a bit of that still touched him, and he was never able to eliminate it completely, perhaps he struggled with it and even held onto some of it intentionally because of his leadership position and the strictness that goes along with it. As for Solomon, he was not able to attain $130 \mathrm{r}$ his wisdom, but as a substitute, he had a firm faith in God and submitted neither to passions nor to the temptation of obscene things. This is nobler than the wisdom of the world and preferable in the sight of God.

As for the Prodromos, ${ }^{803}$ who dwelled in deserts, this man was nothing like him, for he was neither a prophet nor a forerunner and he did not dwell in the desert. However, he did call to faith and display the beauty of worship, and he was well known for this. By my life, he did not baptize a multitude, but he saved many from casting aside and losing baptism, helping them to preserve it by his expenditures and gifts. And afterward, he arrived at the greater, higher baptism, which is not polluted by any dirt or subsequent filth.

From Peter he received a fervent sort of faith, but he nevertheless avoided his cowardice and his denial. ${ }^{804}$ And he resembled Paul in his transformation, though he did not turn from

798. $\mathrm{Z}$ adds: "the ancient."

799. Cf. Genesis 24 .

800. Z omits: "he inherited ... a trick." Cf. Genesis 27.

801. Cf. Genesis 29:15-30.

802. I take this as a reference to the church, specifically the Church of Antioch, thus establishing (as elsewhere in the text) a parallel between Christopher and Christ; cf. Ephesians 5:22-33.

803. A Greek word meaning "forerunner," a title of John the Baptist.

804. Cf. Matthew 26:69-75; Mark 14:66-72; Luke 22:54-62; John 18:15-18, 25-27. 
persecuting religion ${ }^{805}$ but from the confusion of the world and the bondage of the self. And even if he did not make it from Jerusalem to Illyricum to preach ${ }^{806}$-for only Paul made it that far, and he is the one who was snatched up to the third heaven and heard the speech that cannot be expressed ${ }^{807}$-he certainly did not fall short in what he was able to do. There were people whom he supported and strengthened, those he warned and informed, and those he restrained, rebuked, and often rescued and delivered from great difficulties.

\section{[21. Address to the Patriarch]}

But $O$ divine crown, possessor of all purity, especially loved by me and generally venerated by the masses, $O$ you who resembled all of those mentioned here, who loved so many of them and was first to every good deed, receive this discourse from me. $130 \mathrm{v}$ Imagine it as an encomium, or imagine it as an elegy or a consolation, and receive it in your blessed life, even if it is a poor speech, for it is done according to my ability. You did not disdain to run the race for my sake until you could hand me over to that learned divine educator-perhaps you hoped that I would become highly learned, or perhaps you knew beforehand that I, like you, would be lacking ${ }^{808}$ in the sciences. In any case you knew by spiritual knowledge that I was planning to write down your story at some time, so that it might not be forgotten in the course of time. Therefore have compassion on me even now, and pardon me for delaying so long the duty of speaking about you. Do not reject me, now that you are standing in the heavens before the great dais, since I longed-or since I eagerly strove-for you to look upon me and intercede for me. My father relied on you when he made me and my brothers-may God be pleased with all of them ${ }^{809}$-your disciples. And you go to great lengths, asking and begging that my sins be forgiven, that my actions be noble for the remainder of my life, and that I be saved from difficulties and seek whatever will please God and bring me nearer to him.

\section{[22. His Disciples]}

You had numerous followers and helpers, the children whom you fathered in Christ and guided in the way that pleases God. ${ }^{810}$ They were holy branches, spiritual flowers, and pleasing first fruits from our precious, divine town.

Among them was Abba George the Elder, your plant and seedling, who met the holy Emperor Nikephoros and was head of the monastery of St. Symeon the Stylite of Aleppo, the one on the mountain. ${ }^{811}$

\footnotetext{
805. Cf. Acts 9:1-19.
}

806. Cf. Romans 15:19.

807. Z omits: "for only ... be expressed." Cf. 2 Corinthians 12:1-4.

808. For "lacking," Z reads: "excellent."

809. Radilya Allāh 'an al-jamī c.

810. Several of the disciples listed here, along with Ibrāhīm (the author of this text), became translators in Antioch after the Byzantine conquest; see Treiger, "Beginnings," 314-32.

811. Z omits: "the one on the mountain." 
Abba John the Marvelous was worthy of becoming catholicos $131 \mathrm{r}$ over the lands of the East.

Abba Chariton II was the diligent archimandrite of the monastery of St. Symeon the Marvelous, ${ }^{812}$ the one by the sea on the Marvelous Mountain of Lukkām. ${ }^{813}$

Abba Ya ${ }^{\mathrm{c}} \overline{\mathrm{s}} \mathrm{sh}$ the Hermit ${ }^{814}$ struggled upon various pillars of seclusion and performed marvelous feats.

Abba Ephrem, the man of God, ${ }^{815}$ was a hermit who patiently bore the confinement of many cells and did not turn away from his solitary path. He was mainly confined with usor rather, with Christ-in the monastery of St. Gregory the Theologian..$^{816}$

Abba Jeremiah, ${ }^{817}$ his marvelous companion, was head of the monastery of Our Lady the Mother of God al-Jarājima, ${ }^{818}$ which he himself founded on the holy mountain of Lukkām.

The blessed Father Eutykhios, son of Farkhos.

The virtuous Abba Gregory the Elder was head of the monastery of Our Lady the Mother of God Dafnūnā, ${ }^{819}$ and it suffices to mention how famous his virtues were.

I have devoted to each of them an individual account, as they deserve to be remembered, even though I have been far too brief. The simple fact that they were planted by you suffices as a eulogy and commemoration for them. May your intercession and the prayers of all of them save and preserve us, now and ever and until the utmost of all the ages. Amen. May all the people say amen. ${ }^{820}$

812. Z writes that Chariton was the archimandrite of the monastery of St. Saba and lists Symeon the Marvelous as if he were another disciple of Christopher, but Symeon-also known as St. Symeon the Stylite the Younger or as St. Symeon of the Marvelous/Admirable Mountain-lived in the sixth century CE. Alexander Treiger suggests that the text originally listed two Charitons, one abbot of Arshāyā and the other abbot of St. Symeon's monastery; see Treiger, "Beginnings," 323-24.

813. That is, the Black Mountain ("Lukkām" is from the Syriac ūkāmā, meaning "black"), the ancient Amanos, just northwest of Antioch. This monastery is southwest of Antioch, where the southern end of the Lukkām range overlooks the Orontes near its mouth.

814. For "Ya'īsh the Hermit," Z reads: "Ya'īsh of Aleppo." Ya'īsh, along with Jeremiah, is mentioned as a contemporary of St. Timon in several synaxarion entries for March 25; see Joseph-Marie Sauget, Premières recherches sur l'origine et les caractéristiques des synaxaires melkites (XI $-\mathrm{XVII}{ }^{e}$ siècles) (Brussels: Société des Bollandistes, 1969), 367-69.

815. Z omits: "of seclusion ... of God." It therefore combines Ya'īsh and Ephrem into one person.

816. $\mathrm{Z}$ adds "in Bityas." Bityas, also within the Lukkām mountain range, is now called Batıayaz and lies west of Antioch.

817. Jeremiah is mentioned alongside $\mathrm{Ya}^{\mathrm{C}} \mathrm{i}$ sh as a contemporary of St. Timon in several synaxarion entries for March 25; see Sauget, Recherches, 367-69.

818. The Jarājima, known to the Byzantines as Mardaites, were a Christian group living in the mountains near Antioch, often serving as mercenaries for the Byzantines or their enemies; see Nasrallah, "Auteurs," 81-82.

819. Dafnūnā is a reference to Daphnē (modern Harbiye), a suburb south of Antioch with an important history as a pre-Christian religious center; see ibid., 83.

820. Z omits: "May all ... say amen." 


\section{Bibliography}

al-Anțākī, Yahyā b. Sa'īd. "Histoire de Yahya-Ibn-Sa 'ïd d'Antioche, continuateur de Sa ïd-IbnBitriq." Edited and translated by I. Kratchkovsky and A. Vasiliev. Patrologia orientalis 18, no. 5 (1924): 699-833.

al-Anțākī, Yūḥannā 'Abd al-Masīḥ. Maīn al-ḥayāt: al-Markab al-sā'ir fi mīnā' al-najāt, al-ma'rūf bi-Kitāb al-dūlāb. Edited by Habīb Ibrāhīm. 2 vols. Beirut: Markaz al-Turāth al-`Arabī al-Masīhịi, 2020-21.

Bịtāar, Tūmā. Al-Qiddīsūn al-mansiyyūn fi al-turāth al-Anțākī. Duma, Lebanon: 'Ā'ilat al-Thālūth al-Quddūs, 1995.

Bosworth, Clifford Edmund. The Islamic Dynasties: A Chronological and Genealogical Handbook. Edinburgh: University Press, 1967.

Brock, Sebastian. "Syriac Manuscripts Copied on the Black Mountain, near Antioch." In Lingua restituta orientalis, edited by Regine Schulz and Manfred Görg, 59-67. Wiesbaden: Otto Harrassowitz, 1990.

Cahen, Claude. La Syrie du Nord à l'époque des Croisades et le principauté franque d'Antioche. Paris: P. Geuthner, 1940.

Canard, Marius. Histoire de la dynastie des H'amdanides de Jazira et de Syrie. Paris: Presses universitaires de France, 1953.

-—-. Sayf al Daula. Algiers: Editions Jules Carbonel, 1934.

-_- "Une vie du patriarche melkite d'Antioche, Christophore († 967)." Byzantion 23 (1953): 561-69.

Dagron, Gilbert. "Minorités ethniques et religieuses dans l'Orient byzantin a la fin du Xe et au XI ${ }^{\mathrm{e}}$ siècle: L’immigration syrienne.” Travaux et mémoires 6 (1976): 177-216.

Darrouzès, Jean, ed. Épistoliers byzantins $d u X^{e}$ siècle. Paris: Institut français d'études byzantines, 1960 .

Dīk, Ighnāțiyūs. Sīrat al-bațriyark Kharīsțūfürus al-Anțākī șadīq Sayf al-Dawla. Aleppo: n.p., 1997.

Edelby, Néophyte. "Note sur la catholicosat de Romagyris." Proche-Orient chrétien 2 (1952): 39-46.

Forsyth, John Harper. "The Byzantine-Arab Chronicle (938-1034) of Yahyā b. Sa'īd al-Anțākī.” PhD dissertation, University of Michigan, 1977.

Holmes, Catherine. “'How the East Was Won' in the Reign of Basil II.” In Eastern Approaches to Byzantium, edited by Antony Eastmond, 41-56. Aldershot: Ashgate, 2001. 
Ibn al-Athīr, 'Izz al-Dīn. Al-Kāmil fỉ al-tārīkh. Edited by Muhammad Yūsuf al-Daqqāq. Beirut: Dār al-Kutub al-'Ilmiyya, 1987.

Ibrahim, Habib. "Liste des vies de saints et des homélies conservées dans les Ms. Sinaï Arabe 395-403, 405-407, 409 et 423." Chronos 28 (2018): 47-114.

Ibrāhīm b. Yūḥannā. “Zhitie antiokhiǐskogo patriarkha Khristofora.” Translated by S. A. Moiseeva. In Araby-Khristiane v istorii i literature Blizhnego Vostoka, edited by N. G. Golovnina, 28-61. Moscow: PSTGU, 2013.

Kennedy, Hugh. The Prophet and the Age of the Caliphates: The Islamic Near East from the Sixth to the Eleventh Century. London: Routledge, 2016.

Leo the Deacon. The "History" of Leo the Deacon: Byzantine Military Expansion in the Tenth Century. Translated by Alice-Mary Talbot and Denis F. Sullivan. Washington, DC: Dumbarton Oaks Research Library and Collection, 2005.

Michael the Syrian. Chronique. Edited and translated by J.-B. Chabot. 4 vols. Brussels: Culture et Civilisation, 1963.

Moiseeva, Sofia A. Arabskaia mel'kitskaia agiografiia IX-XI vekov. Moscow: PSTGU, 2015.

---. "The Early Melkite Arabic Hagiography (IXth-XIth Centuries): Evolution of the Literary Style." Parole de l'Orient 39 (2014): 33-56.

Mugler, Joshua. "Ibrāhīm ibn Yūḥannā and the Translation Projects of Byzantine Antioch." In Patristic Literature in Arabic Translations, edited by Barbara Roggema and Alexander Treiger, 180-97. Leiden: Brill, 2020.

-C-. "A Martyr with Too Many Causes: Christopher of Antioch (d. 967) and Local Collective Memory.” PhD dissertation, Georgetown University, 2019.

Nasrallah, Joseph. "Deux auteurs melchites inconnus du $\mathrm{X}^{\mathrm{e}}$ siècle." Oriens christianus 63 (1979): 75-86.

-_-. "La liturgie des patriarcats melchites de 969 à 1300." Oriens christianus 71 (1987): 156-81.

Noble, Samuel. "A Byzantine Bureaucrat and Arabic Philosopher: Ibrāhīm ibn Yūḥannā al-Anțākī and His Translation of On the Divine Names 4.18-35." In Caught in Translation: Studies on Versions of Late Antique Christian Literature, edited by Madalina Toca and Dan Batovici, 276-312. Leiden: Brill, 2020.

Roberts, Alexandre M. Reason and Revelation in Byzantine Antioch: The Christian Translation Program of Abdallah ibn al-Fadl. Oakland: University of California Press, 2020.

Sauget, Joseph-Marie.Premières recherches sur l'origine et les caractéristiques des synaxaires melkites (XI $-X V I I^{e}$ siècles). Brussels: Société des Bollandistes, 1969. 
Treiger, Alexander. "The Beginnings of the Graeco-Syro-Arabic Melkite Translation Movement in Antioch." Scrinium 16 (2020): 306-32.

-_-. "Sinaitica (1): The Antiochian Menologion, Compiled by Hieromonk Yūhannā 'Abd al-Masịh (First Half of the 13th Century)." Khristianskīi Vostok" 8 (2017): 215-52.

Zayat, Habib. Khabāyā al-zawāyā fĩ tārīkh Șaydnāyā. Harissa, Lebanon: Imprimerie de Saint Paul, 1932.

- - "Vie du patriarche melkite d'Antioche Christophore ( $†$ 967) par le protospathaire Ibrahîm b. Yuhanna: Document inédit du X siècle." Proche-Orient chrétien 2 (1952): 11-38, 333-66. 